\title{
The Role of Thin Slices of Behavior and Incentive Compensation in Employee Selection Decisions
}

Andrew Michael Carnes

Follow this and additional works at: https://researchrepository.wvu.edu/etd

\section{Recommended Citation}

Carnes, Andrew Michael, "The Role of Thin Slices of Behavior and Incentive Compensation in Employee Selection Decisions" (2015). Graduate Theses, Dissertations, and Problem Reports. 5316.

https://researchrepository.wvu.edu/etd/5316

This Dissertation is protected by copyright and/or related rights. It has been brought to you by the The Research Repository @ WVU with permission from the rights-holder(s). You are free to use this Dissertation in any way that is permitted by the copyright and related rights legislation that applies to your use. For other uses you must obtain permission from the rights-holder(s) directly, unless additional rights are indicated by a Creative Commons license in the record and/ or on the work itself. This Dissertation has been accepted for inclusion in WVU Graduate Theses, Dissertations, and Problem Reports collection by an authorized administrator of The Research Repository @ WVU.

For more information, please contact researchrepository@mail.wvu.edu. 


\title{
The Role of Thin Slices of Behavior and Incentive Compensation in Employee Selection
} Decisions

\author{
Andrew Michael Carnes \\ Dissertation submitted \\ to the College of Business and Economics \\ at West Virginia University \\ in partial fulfillment of the requirements for the degree of \\ Doctor of Philosophy in Business Administration \\ Concentration in Management \\ Joyce T. Heames, Ph.D., Chair \\ David D. Dawley, Ph.D. \\ Mark B. Gavin, Ph.D. \\ Jeffery D. Houghton, Ph.D. \\ Timothy P. Munyon, Ph.D., Outside Committee Member \\ Department of Management
}

Morgantown, West Virginia
2015

Keywords: employee selection, decision making, thin slices of behavior, fuzzy trace theory, compensation

Copyright 2015 Andrew Carnes 


\begin{abstract}
The Role of Thin Slices of Behavior and Incentive Compensation in Employee Selection Decisions
\end{abstract}

Andrew M. Carnes

Despite notable research attention, past studies addressing decision stability have provided conflicting recommendations regarding the stability and impact of initial impressions in an interview context. As a result, this dissertation focused on the integration of literature addressing decision making, employee selection, and influence processes in an attempt to provide both a theoretical and empirical foundation for future research addressing initial impressions throughout the interview process. Utilizing fuzzy trace theory and the thin slices of behavior literature, it was hypothesized that impressions formed at career fairs and at the beginning of interviews would account for significant variance in final impressions of employability even after considering interview interactions. In addition, motivational and cognitive components were added to the model to assess the impact of incentive compensation and decision confidence on employability rating stability. Using data from a simulation comprised of 28 recruiters and 229 applicants, results suggest that initial impressions formed at the beginning of the interview make a substantive impact on final impressions. However, impressions formed at the career fair do not appear to impact final impressions without considering the interactive effects of decision confidence. Hypotheses proposing that decision confidence would moderate linkages between initial impressions formed at the beginning of the interview and both interview scores and final impressions were not supported. In addition, results suggest that incentive compensation did not impact decision stability with the caveat that the manipulation checks for the incentive compensation manipulation were not significant. In all, these results suggest that initial impressions have a substantive impact on final impressions of employability and that decision confidence may increase decision stability over time. These findings encourage future research assessing the task-relevance of initial impressions, potential biases contained within first impressions, and the impact of initial impressions in unstructured interviews. 
To Lauren, Emma, and Future Child(ren) 


\section{ACKNOWLEDGEMENTS}

It goes without saying that I could not have completed this dissertation without the support of many individuals. First, I would like to wholeheartedly thank my Dissertation Chair, Joyce Heames, and my methods guru, Mark Gavin, for your unwavering support and guidance throughout this process. You both provided invaluable expertise, procedural guidance, and emotional support during this grueling process. I am forever grateful to both of you for these contributions and I simply could not have done this without you.

Next, my dissertation committee provided invaluable support ranging from substantive guidance for experimental design to strategic comical relief. Tim Munyon, you were my primary role model when I decided to pursue my $\mathrm{PhD}$ and your guidance in all areas of my career have been immeasurable. I cannot thank you enough for your contribution to the theory and design of this study as well as your constant encouragement from the first day of my doctoral training. Jeff Houghton, thank you for your continual support and keen insights regarding the design of this study and minimizing its limitations. I also appreciate your laborious efforts in providing editorial support for this massive document. David Dawley, I am extremely grateful for your "big picture" viewpoint throughout this project and your comedic contributions to our meetings. We would be a myopic and somewhat boring group without you. In addition, I would like to thank Bob Vandenberg for his troubleshooting help regarding the convergence of my multilevel SEM model.

I would be remiss not to mention other valuable members of the faculty at West Virginia University that have aided in my development as a scholar including Abhishek Srivastava, Miles Zachary, Jennifer Sexton, Matthew Marvel and Jun Xia. I would like to provide special thanks to Jodi Goodman and Edward Tomlinson for your intensive development of my research ideas and skills that ultimately led to publication and overall improvement. I would also like to thank the Institutional Review Board at West Virginia University for their service and support when designing this study and ensuring its integrity. Finally, I am very thankful for the support provided by my fellow doctoral students with special thanks to Curtis Sproul and Kevin Knotts. You all helped to maintain my sanity, which was no small task. 
On a personal note, I am so very grateful for all of my friends and family who supported me along the way. First, my parents Greg and Susan Carnes deserve a thank you note that would be longer than this dissertation itself. Your support through life in teaching me the meaning of hard work and achievement combined with your love and sacrifice for me simply cannot be put into words. Rest assured that I am eternally grateful for your efforts in making me the man that I am today. In addition, I am extremely grateful for the support of my brother Tony Carnes, my grandparents Bill Allen, Jeanette Allen, Rex Carnes, and Kay Carnes, and the Wagner family throughout this grueling process. I would also like to thank my church families that have provided unwavering support throughout the years. Members of Myers Memorial United Methodist Church, Cullowhee United Methodist Church, and Suncrest United Methodist Church have always been so welcoming and supportive to my family and myself. I would like to especially thank David Reeves, Jeff Calvert, Eddie Shaw, and Mike Rhodes for your efforts toward shaping me into the man that I am today.

I saved my most important supporters for last. As the old saying goes, "behind every great man is a great woman." I disagree slightly and would rewrite this statement to read "beside every great man is a great woman." Lauren, I consider this dissertation to be just as much your accomplishment as it is mine. Your sacrifice, love, and understanding over the past four years go beyond words and beyond measure. I could never repay you for contributions to this dissertation, but please know that it means the world to me. To my daughter Emma, you are simply the light of my life. I never knew that I could come to love bows, ruffles, and pink paisley outfits until I met you. I love you so much and I thank you for the motivation that you provided to finish this dissertation. Finally, but most importantly, I thank the Lord for support and guidance through this process as there is simply no way that I could have done it alone. Rom. 8:38-39. 
Table of Contents

Chapter 1: Introduction 1

1.1 Thin Slices of Behavior $\quad 1$

1.2 Intuitive Decision Making and Fuzzy Trace Theory 2

1.3 Incentive Compensation and Decision Making 4

1.4 Conclusion $\quad 5$

Chapter 2: Literature Review 6

2.1 Decision Making $\quad 7$

2.1.1 Perspectives from Mathematical Psychology 7

$\begin{array}{ll}\text { Logicism and Schemata } & 7\end{array}$

Formalism and Information Processing 9

$\begin{array}{ll}\text { Intuition } & 11\end{array}$

2.1.2 Integrating Traditional Perspectives 13

$\begin{array}{ll}\text { The Dual Processing Perspective } & 13\end{array}$

2.1.3 A "New” Perspective: Fuzzy Trace Theory 15

$\begin{array}{ll}\text { Gist Extraction } & 17\end{array}$

Fuzzy to Verbatim Continuum 18

The Fuzzy Processing Preference 19

Reconstruction of Short-Term Memory $\quad 21$

2.2 Talent Acquisition and Employment Interviews 23

2.2.1 Interview Structure $\quad 26$

Developing a Nomenclature for Interview Structure 28

Types of Structured Interview Questions 30

2.2.2 Pre-Interview Interactions and Impressions 32

2.2.3 Integration with the Decision Making Literature 34

2.2.4 Applicant Factors Influencing the Selection Process 38

2.3 Thin Slices of Behavior: Learning a Lot from a Little 41

2.3.1 Accuracy of Thin Slices $\quad 44$

2.3.2 Effectiveness for Predicting Different Traits 45

2.3.3 Thin Slicing in an Organizational Context 46 
2.4 Compensation and Pay for Performance $\quad 47$

2.4.1 Economic Perspectives 48

2.4.2 Psychological Perspectives - Can Compensation Motivate? $\quad 49$

$\begin{array}{ll}\text { Cognitive Evaluation Theory } & 50\end{array}$

2.4.3 Psychological Perspectives - Incentive Compensation 52

Reinforcement Theory $\quad 52$

Expectancy Theory $\quad 54$

Equity Theory $\quad 56$

Goal Setting Theory $\quad 57$

2.4.4 Incentive Structure and Distribution

$\begin{array}{ll}\text { Outcome Measures } & 60\end{array}$

Temporal Sequencing $\quad 61$

Individual and Team Incentives $\quad 62$

Forms of Incentives $\quad 63$

$\begin{array}{ll}\text { Incentive Intensity } & 64\end{array}$

Chapter 3: Hypothesis Development 66

3.1 Thin Slices of Behavior in Employment Interviews 66

3.2 The Effect of Confidence on Decision Stability 72

3.3 The Impact of Incentive Compensation $\quad 74$

Chapter 4: Method 79

$\begin{array}{ll}\text { 4.1 Participants } & 79\end{array}$

4.2 Experimental Design $\quad 79$

4.3 Compensation Manipulation $\quad 81$

$\begin{array}{ll}4.4 \text { Measures } & 82\end{array}$

4.4.1 Perceptions of Employability 82

4.4.2 Rating Confidence $\quad 82$

4.4.3 Structured Interview Scores $\quad 83$

4.5 Control Variables $\quad 83$

$\begin{array}{ll}\text { 4.5.1 Gender Congruence } & 83\end{array}$ 
4.5.2 Race Congruence $\quad 84$

4.5.3 Applicant Objective Qualifications $\quad 84$

4.6 Analytic Strategy

Chapter 5: Results 90

5.1 Descriptive Statistics and Correlations 90

5.2 Baseline Multilevel Structural Equation Model 90

5.3 Strength of Second Impressions 93

5.4 The Effect of Decision Confidence on Decision Stability 94

5.5 The Effect of Incentive Compensation on Decision Stability 96

$\begin{array}{ll}\text { 5.6 Supplemental Analyses } & 100\end{array}$

Chapter 6: Discussion 105

6.1 Major Findings 105

6.1.1 The Base Model: Employability Rating Stability 105

6.1.2 Relative Importance: Second Impressions vs. Interview Scores 106

6.1.3 Decision Confidence and Employability Rating Stability 106

6.1.4 Incentive Compensation and Employability Rating Stability 107

$\begin{array}{ll}\text { 6.2 Contributions of the Dissertation } & 108\end{array}$

6.3 Strengths and Limitations of the Research Design 111

6.3.1 Strengths 111

6.3.2 Limitations 112

6.4 Implications for Research 116

6.5 Directions for Future Research 119

6.6 Implications for Practice 123

$\begin{array}{ll}6.7 \text { Conclusion } & 125\end{array}$

APPENDIX A: Structured Interview Form 127

APPENDIX B: Measurement Scales 131

APPENDIX C: Institutional Review Board Approval Letter 133

APPENDIX D: Incentive Compensation Scripts 134 
APPENDIX E: Fixed Compensation Condition Employment Agreement

APPENDIX F: Incentive Compensation Condition Employment Agreement 
List of Tables

Table 1: Dual Processing Terminology Disagreement 182

Table 2: Responses to Memory Probes 183

Table 3: Thin Slicing Empirical Review 184

Table 4: Means, Standard Deviations, and Intercorrelations Among Study Variables 186

Table 5: Baseline Model Structural Path Results 187

Table 6: Hypothesis 7 Constrained Model Structural Path Results 188

Table 7: Sattora-Bentler Scaled $X^{2}$ Difference Test for Hypothesis $7 \quad 189$

Table 8: First Impression $\rightarrow$ Second Impression Confidence Moderation (H8a) 190

Table 9: Second Impression $\rightarrow$ Interview Score Confidence Moderation (H8b) 191

Table 10: Second Impression $\rightarrow$ Final Impression Confidence Moderation (H8c) 192

Table 11: Compensation Baseline Structural Path Results 193

Table 12: Full Compensation Moderation Structural Path Results 194

Table 13: Sattora-Bentler Scaled $X^{2}$ Difference Test for Full Compensation Moderation 195

Table 14: Initial Impressions $\rightarrow$ Second Impressions Compensation Moderation (H9a) 196

Table 15: Sattora-Bentler Scaled $X^{2}$ Difference Test for Initial Impressions $\rightarrow$ Second

Impressions (H9a) Compensation Moderation 197

Table 16: Second Impressions $\rightarrow$ Interview Score Compensation Moderation (H9b) 198

Table 17: Sattora-Bentler Scaled $X^{2}$ Difference Test for Second Impressions $\rightarrow$ Interview

Score (H9b) Compensation Moderation 199

Table 18: Second Impressions to Final Impressions Compensation Moderation (H9c) 200

Table 19: Sattora-Bentler Scaled $X^{2}$ Difference Test for Second Impressions $\rightarrow$ Final

Impressions (H9c) Compensation Moderation 201

Table 20: Effect Sizes for the Indirect Effect of Second Impressions on Final Impressions $\begin{array}{ll}\text { through Interview Scores } & 202\end{array}$

Table 21: Manipulation Check T-Test Results 203

Table 22: Hypothesis Results 204 


\section{List of Figures}

Figure 1: Conceptual Continuum of Information Processing 206

Figure 2: Huffcutt \& Arthur's (1994) Interview Structure Nomenclature 207

Figure 3: Expectancy Theory Flowchart 208

Figure 4: Hypothetical Model 209

Figure 5: Overall Model 210

Figure 6: First Impression x Confidence Interaction Plot (H8a) 211

Figure 7: Hypothesis Results $\quad 212$

Figure 8: Fixed Compensation Recruiter Rating Stability 213

Figure 9: Incentive Compensation Recruiter Rating Stability 214 


\section{CHAPTER 1: INTRODUCTION}

Few scholars dispute the strategic and financial impact that personnel decisions make on the functioning and profitability of an organization (e.g. Dipboye, 1984; Huffcutt, 2011). That being said, there is room for improvement in the literature addressing the processes behind personnel decision making. For example, theoretical, empirical, and meta-analytic evidence exists to address the link between individual factors (such as traits, states, and moods) and both selection decisions (e.g. Huffcutt et al., 2011) and job performance (e.g. Judge et al., 2002; Barrick \& Mount, 1991; etc.). However, the current literature is fractured regarding the mechanisms through which these traits impact decision processes. It could be fruitful to address the way in which managers and recruiters make personnel decisions to understand error and biases in the selection process. This dissertation will address a gap in the existing literature that concerns the process by which these decisions are made and the cognitive systems that are activated when making decisions. Given the financial and legal impact of poor personnel decisions, this research will also fill a practical gap in addition to its academic value.

\subsection{THIN SLICES OF BEHAVIOR}

An important factor that has been left largely unaddressed in the organizational decisionmaking literature is the temporal aspect behind the acquisition of information used in making decisions. At current, much of the literature addressing personal interaction between recruiters and applicants examines structured and unstructured interviewing (e.g. Huffcutt, 2011).

However, the existing literature regarding thin slices of behavior could shed light on this topic by identifying the amount of interaction time necessary for an individual to obtain the information necessary to make an informed personnel decision. Thin slices of behavior address one's ability to find patterns in events based only on narrow periods of exposure of as little as five seconds 
(Ambady \& Rosenthal, 1992). The primary assertion of this stream of literature suggests that a rater's accuracy when judging attributes of another individual are very similar when comparing very short periods of exposure to very long periods of exposure (Bernieri, Gillis, Davis, \& Grahe, 1996). Empirical evidence even suggests that individuals might be better at detecting deception in short interactions with decreasing accuracy as time passes (Albrechtsen, Messiner, \& Susa, 2009). This combined evidence suggests that recruiters may be able to make accurate hiring decisions within the first few minutes of a traditional interview. It also may be helpful to identify the temporal point at which an interview reaches a point of diminishing returns with regards to the amount of useful information that is captured for making selection decisions. Determining this point of diminishing returns could help to streamline the interview process by reducing the length of interview without sacrificing decision quality. In this way, the thin slicing perspective could inform the employee selection literature by determining the temporal point at which informed selection decisions can be made as well as the impact of subsequent interactions on initial impressions. This existing evidence and its applicability to employment interviews informs the following research questions:

1. Can decision-makers accurately identify factors that lead to success by utilizing thin slices of behavior?

2. Do thicker slices of behavior provide useful information beyond that which is collected during initial observation of thin slices?

\subsection{INTUITIVE DECISION MAKING AND FUZZY TRACE THEORY}

The existing literature addressing decision-making in organizations suggests that individuals build cognitive frameworks, commonly referred to as schemas, to aid in making 
decisions (Piaget, 1926). The literature also suggests that individuals use these frameworks to consciously analyze multiple pieces of information with which to make an informed selection decision (e.g. Brainerd \& Reyna, 1990). This would suggest that managers and recruiters use their experience to make more accurate personnel decisions by building cognitive schemas that aid in making informed decisions. The primary problems inherent in this perspective are a lack of evidence that individuals actually engage in these processes (Brainerd \& Reyna, 1990) and evidence showing the potential for bias during information processing (e.g. Staw, 1981). Another point of view suggests that individuals simplify the decision-making process in a slightly different fashion. The fuzzy trace perspective suggests that individuals compress the information that they are presented primarily by storing the gist of the information that they are presented rather than intricate verbatim details (Brainerd \& Reyna, 1990). Gist representations are bottomline understandings of past events, while verbatim representations contain information about specific details surrounding the event (Reyna \& Brainerd, 1995a). One of the main advantages to storing gist information is the assertion that they utilize much less space in working memory (e.g. Thompson, 2009), therefore allowing for simplified information processing. An example of how this process works is evidenced by a recruiter's storage of information regarding the appearance of a candidate. Verbatim details could include the color match between the applicant's shirt and tie, whether the shirt was pressed or wrinkled, the presence or absence of well-shined shoes. On the other hand, a gist representation of the situation would simply boil down to a statement such as "the applicant was dressed in business-professional attire."

In addition to the above, fuzzy trace theory suggests that individuals tend to operate on the fuzzy end of the gist-verbatim continuum (Reyna \& Brainerd, 1995a). This implies that individuals prefer storing information in gist form and that they choose to utilize gist information 
more often than verbatim information when making decisions. This portion of fuzzy trace theory is important in an organizational decision-making context in that decision-makers might lose valuable information by choosing gist memory storage rather than verbatim. On the other hand, storing information via gist processing might help to more accurately evaluate larger quantities of applicants or employees without losing important information. These two streams of research lead to the following research questions for further exploration:

3. Do gist representations formed during thin slices of behavior account for unique variance in interview scores or selection decisions?

4. Can gist representations be reconstructed for future usage in cognitive processing?

\subsection{INCENTIVE COMPENSATION AND DECISION MAKING}

While the literature addressing employee compensation is quite comprehensive with regards to executive decision making (e.g. Gomez-Mejia \& Wiseman, 1997; Pepper \& Gore, in press), managerial performance (e.g. Rajan \& Reichelstein, 2009), and non-managerial performance (e.g. Jenkins, Mitra, Gupta, \& Shaw, 1998), very little research has addressed the impact of incentive compensation on non-executive decision making. Extending this line of inquiry is crucial given that non-executives often make decisions that are strategic in nature, but their compensation structure does not contain the risk management provisions typically included for executives. For example, recruiters and hiring managers make routine decisions that ultimately impact the firm's human capital and ultimately create or hinder a competitive advantage. In addition, many recruiters already receive pay for performance incentives (e.g. Lipp, Stanley, \& Lariviere, 2007) although very little theorizing or empirical testing directed toward this issue. For this reason, exploring the impact of incentive compensation on the 
cognitive processes and decision outcomes of non-executive employees could contribute to both research and practice.

5. How does incentive compensation impact the decision processes of non-executive decision makers?

\subsection{CONCLUSION}

In short, this manuscript aims to address the process by which personnel decisions are made and the specificity of the information that decision makers use when making decisions. Understanding the intricacies of this process is critical to research in that we can begin to understand the specific reasons for error and bias in the decision-making process and begin to address ways in which to improve the process. In addition, understanding the impact of incentive compensation on the decision process should highlight the utility or detriment of utility of such practices for improving decision processes. This inquiry also generates practical implications in that managers can understand how decision-making processes occur and streamline the process without losing important variance of interest. 


\section{CHAPTER 2: LITERATURE REVIEW}

Anecdotal and empirical evidence tells us that individuals at all levels of the organization make decisions daily that can dramatically impact the financial performance and ultimate survival of the organization (e.g. Gomez-Mejia \& Wiseman, 1997). For example, executives might make decisions about divesting subsidiaries while middle managers often make decisions about daily operating procedures and non-managerial employees make decisions about whether or not to engage in counterproductive behaviors. In addition, decision-making underlies many of the constructs that are often discussed in the management literature, such as turnover (deciding to leave the organization), organizational citizenship behaviors (deciding to perform work that will not be formally recognized), and absenteeism (deciding not to attend). Many of these literatures give little consideration to the decision-making processes that are at work behind employee action without accounting for the irrational intricacies of human cognitive processing (e.g. Rilling \& Sanfey, 2011).

Given the ubiquity of decision-making in organizations, it is important to consider the processes involved in making decisions, the time necessary to make accurate decisions, and the information used by decision-makers. The foundations of the current decision-making literature are rooted in mathematical psychology (Brainerd \& Reyna, 1990) that dates back to the midnineteenth century. These perspectives present the assumptions behind human decision-making and set the stage for analyzing the processes by which humans make decisions.

Early research in decision-making sought out mathematical metaphors to describe the processes that humans employ when making decisions. The first such perspective is the logical view that was developed by Frege (1884) and suggests that "doing logic" was similar to "doing 
mathematics." This premise is based on deductive logic that sets up foundational premises and derives conclusions by comparing the premises to deterministic rules (Brainerd \& Reyna, 1990). Other scholars, such as Russell (1903) and Whitehead and Russell (1910-1913) advanced this cause by providing anecdotal evidence that humans use logical patterning to make decisions. Another camp promulgated by Hilbert (formalism; 1928) suggests that individuals engage in sequential processing, which led to the assertion that human cognition incorporates higher-order factors. Finally, another group of scholars rejected the foundations upon which the logical and formalistic perspectives were based by suggesting that individuals make quick decisions based on intuition (Brouwer, 1952; Heyting, 1959). Below, each of these perspectives will be discussed to highlight the merits and drawbacks of each perspective with special emphasis on how they interact with one another.

\subsection{DECISION MAKING}

\subsubsection{Perspectives from Mathematical Psychology}

Logicism and Schemata. Arguably the most prominent work that was developed from the logical line of reasoning is Piaget's (1926/1960) idea of decision-making schemata. Schemata are used to organize knowledge about a particular concept for use in identifying category membership (Sims \& Lorenzi, 1992). These structures allow individuals to provide a programmed response when they perceive that a stimulus is similar to that experienced in a previous situation. For example, an investor might choose not to invest in a particular stock if he or she recognizes that the company is a high tech startup based on a past experience in which money was lost on a high tech startup failure. As this concept developed, for main types of schemata emerged: person, event, role, and self-schemata (Sims \& Lorenzi, 1992). Person 
schemata (attributions as to the personality of another individual) and role schemata (expectations for an individual in a given role) are the most important in a personnel decision making context in that they inform the decision maker's perception as to the target individual's competence and fit with the organization. The primary assertion of the schema perspective is that decision makers compare information gleaned from the target individual to that contained within cognitive structures that identify positive and negative performance predictors. In essence, decision making schemata would be used in a selection context by comparing attributes of the target individual to attributes of positive and negative performers to determine whether or not the target individual would become a successful employee.

The above information addressing the utility of schemata makes logical sense, but existing research provides conflicting information as to the accuracy of schemata when describing the ways in which individuals make decisions. On one hand, it appears that Piaget's theory is supported by a substantial amount of empirical evidence (e.g. Smedslund, 1969; Dean, Chabaud, \& Bridges, 1981). However, closer inspection reveals that many of these early experiments do not actually require participants to adhere to hypothetico-deductive processing. For example, Smedslund (1969) suggested that complex inferences are necessary to describe even the most simplistic processes when addressing sequential decision making via logic. He also suggested that most research addressing mathematical logic is flawed due to the lack of control for individual perceptions, misunderstanding, and forgetfulness throughout the longitudinal process of making sequential logical decisions. In addition, Dean and colleagues (1981) showed that research that forces participants to use specific categories or types of categories may fail to produce accurate results. Later work by Anderson (1983) also casts doubt on the simplicity of the logical perspective by suggesting that individuals often make sequential 
decisions without considering prior information (thereby leading to illogical decisions) and that the goals of the process are often discovered during decisions making rather than the binding effect of preexisting rules and goals as suggested by Piaget (1926/1960). Other common criticism of the Piagetian perspective include humans' lack of capability for being purely logical and repeated empirical studies that disprove Piaget's proposition that binary propositional logic can be considered as the calculus of mental cognition (e.g. Brainerd \& Reyna, 1990).

Formalism and Information Processing. In response to the shortcomings of the logical perspective, Hilbert (1923) introduced the formalistic perspective as another early perspective that attempted to describe the mathematics behind human decision making. Hilbert and his associates suggested that cognition is actually a result of individuals associating symbols with rituals rather than building cognitive schemata with which to make decisions (Brainerd \& Reyna, 1990). In essence, this perspective considers the human mind to be a "computer" that manipulates symbolic mental representation based on past experiences and rituals (Shannon, 1987). Information processing is portrayed as a conscious form of cognition which rests on four fundamental assumptions: thinking, analysis of stimuli, situational modification, and obstacle evaluation.

According to this perspective, thinking involves the collection and evaluation of external stimuli for encoding and storing information surrounding the decision itself (Bourne, Dominowski, \& Loftus, 1979). It is the crux on which the information processing perspective rests given its analogy that information processing is guided by preexisting routines that are similar to those contained within computer programs (Lord \& Maher, 1990). These routines for processing information are stored in long term memory and are recalled when needed to compare current stimuli to those experienced in the past and to evaluate the potential impact of obstacles 
on various courses of action. The short-term memory is also important to the process in that it allows the individuals to hold and process newly experienced stimuli for comparison with those in long-term memory. However, the utility of short-term processing is limited by earlier findings suggesting that individuals can only retain approximately seven symbols for twenty seconds at a time without transitioning the information into long term memory that is less accessible (Miller, 1956). The computer analogies for these systems portrays short-term memory as RAM that actively processes information, while long-term memory acts as a hard drive that stores information for use in future processing.

In short, research addressing the information processing perspective provides mixed support regards to the frequency with which individuals utilize information processing procedures as well as the accuracy of the conclusions that result from detailed information processing. First, it appears that individuals do often engage in complex, sequential decision making processes. Evidence supporting this conclusion comes from numerous early experiments that display the use of both short-term and long term memory, effortful processing, and selective information retention (e.g. Shallice \& Warrington, 1970; Cowan, 1988). However, there is also evidence to suggest that individuals engage in "automatic" processing that is non-conscious and is not sequential (Shiffrin \& Schneider, 1977). Although some consider this "automatic" processing to be a component of the information processing perspective, it is clear that it fits more closely with the intuitive perspective that will be discussed later.

Second, a substantial amount of empirical evidence shows that information processing often results in biased conclusions. A prime example of this effect is displayed in Kahneman \& Tversky's (1979) prospect theory. The theory and its subsequent empirical support shows that individuals vary in the amount of risk that they are willing to bear based on their reference point 
(e.g. Whyte, 1993). In short, individuals are willing to take more risk when they perceive the situation as a loss overall than when the situation is set in the context of a gain regardless of objective reality. Another example of biased decision making is inherent in the escalation of commitment literature. Escalation of commitment occurs when an individual continues to commit resources to a given course of action despite losses and a meager possibility of success (Staw, 1981). Field studies addressing this effect show that complex cognitive processing can lead to bias in the decision outcome due to the consideration of personal image concerns in an organizational principal-agent relationship (e.g. McNamara, Moon, \& Bromiley, 2002;

Sleesman, Conlon, McNamara, \& Miles, 2012).

Intuition. Despite the above success in the information processing realm, researchers also explored the possibility that decision-making is not always conscious and calculated (e.g. Brainerd \& Reyna, 1990). Early research addressing intuitionism was promulgated by Brouwer (1952) and Heyting (1959) and asserted that logicism and formalism failed to capture the innate imaginativeness of human mathematical cognition. Instead, Brouwer (1952) suggested that intuition consists of a generic item of information, often called a basal entity, which can be combined with an invention rule to create new knowledge from the reformulation. Inside of this paradigm, theorists suggest that every sentence that humans utter is an invention that results often from quick, effortless thinking. Its supporters view this perspective as superior due to the fact that it allows mental cognition to range freely along many different paths, rather than the constriction suggested by formalism and logicism (e.g. Heyting, 1959; Brainerd \& Reyna,1990). In addition, they assert that individuals tend to manipulate only the surface meaning of previously stored information, rather than moving on a rigid path from fixed, preconceived notions to inescapable conclusions (Brainerd \& Reyna, 1990). 
As can be seen above, those taking the intuitive perspective saw evolution, impreciseness, and fluidity where formalists and logicists saw immutability, rigor, and structure. Keeney and Raiffa (1993) provided support for the intuitive perspective by exposing the salience of intuitive decision making in organizations by noting that this type of decision making is adopted more often that it deserves. In addition, they note that individuals tend to hide their use of intuition as it is naively simple and rarely passes the test of "reasonableness." In the empirical realm, Ford, Schmitt, Schechtman, Hults, and Doherty (1989) provided an empirical review of 45 studies to show that noncompensatory (failure to consider all relevant available information) strategies were used more often than compensatory strategies when making decisions. In addition, their review showed that compensatory strategies are only used when the number of alternatives and dimensions were very small.

In addition to the above, there are several constructs based on the intuitive perspective that enjoy widespread empirical support. First, in the economics literature, the concept of satisficing is very prevalent. Satisficing is the assertion that individuals search through available alternatives until an acceptable option is discovered (Simon, 1956). This is contrasted with optimal decision making in which individuals weigh all available alternatives to make the best possible decision. In addition, the literature also distinguishes between weak satisficing, in which individuals engage in multiple cognitive steps with less completeness and more bias than in optimal decision making, and strong satisficing, which involves selecting the first reasonable alternative available without memory searching or integrating information (Brown, 2004). In terms of the intuitive perspective, this suggests that individuals utilizing strong satisficing only exert the amount of cognitive effort necessary to make a "good" decision even if it is not the best 
decision. Fuzzy trace theory (Brainerd \& Reyna, 1990), which will be further discussed later, is another viewpoint that provides theoretical and empirical support for the intuitive perspective.

\subsubsection{Integrating Traditional Perspectives}

The Dual Processing Perspective. In sum, support for both the intuitive and information processing perspectives exist, but it appears that both processes might coexist and be utilized in human cognition and decision-making. In response to this conflicting information, numerous scholars have proposed that dual processing occurs. From this perspective, cognitive functioning follows two different paths termed System 1 and System 2 (e.g. Stanovich, 1999). The primary factors used to differentiate these processes are the level of consciousness and explicitness of processing. System 1 refers to processing that is quick, unconscious, and implicit, while System 2 refers to processing that is conscious, explicit, and controlled (St. B. T. Evans, 2008). The important modification that the dual processing perspective provides is that both the intuitive and formalistic perspectives can coexist and even occur simultaneously.

Despite the seemingly simple differentiation between these two types of processing, the literature is highly fractured regarding the specifics of each system. As shown in Table 1 below, there is very little agreement as to the terminology that should be used for each system. As a result, many scholars resort to the System 1 and System 2 terms introduced by Stanovich (1999) as they can act as a clustering system comprised of multiple factors rather than precise terminology (e.g. St. B. T. Evans, 2008). However, it does appear that there is some underlying agreement regarding the nature of each cluster. System 1 appears to occur very rapidly with little to no cognitive input on the part of the decision maker (e.g. Toates, 2006; Reber, 1993). In addition, most contributors in Table 1 seem to agree that the System 1 process is automatic and 
non-conscious (e.g. Schneider \& Schiffrin, 1977; Wilson, 2002). These factors are all common to the intuitive perspective discussed earlier. In contrast, System 2 processes listed in Table 1 appear to fall in the area of the formalistic inquiry. Descriptions such as systematic (Chaiken, 1980), analytic (Nisbett, Peng, Choi, \& Norenzayan, 2001), rule-based (Sloman, 1996), and conscious (Wilson, 2002) provide support to this notion. For this reason, it appears that new information might result from the integration of the mathematical psychology and dual processing literatures.

Insert Table 1 About Here

As noted in the dual processing literature, there are benefits and drawbacks of utilizing System 1 and System 2. System 1 is advantageous due to the fact that it can handle larger amounts of information and that it can provide support for System 2 functioning (Epstein, 1994). In addition, System 2 provides its own advantages in its protection against bias and impulsivity errors (e.g. Frederick, 2005). For example, Dijksterhuis, Bos, Nordgren and von Baaren (2006) provided evidence to suggest that individuals can make accurate decisions without engaging in conscious information processing. However, Frederick (2005) found that individuals are prone to making intuitive errors when presented with seemingly easy numerical calculations. In addition, St. B. T. Evans (2003) discovered that bias is inherent in System 2 processing in that individuals are more likely to side with their existing beliefs even if they do not follow a logical pattern.

Given these conflicting data, scholars set out to test the idea that both System 1 and System 2 processes might be able to operate simultaneously. In general, results support the 
notion that System 1 can operate on its own, while it is also used as a support mechanism for System 2. Evidence supporting the standalone vitality of System 1 is provided by Eisendardt (1989), Hitt, Keats, and DeMarie (1998), and Perlow, Okhuysen, and Repenning (2002), who all showed the utility of System 1 for making quick and reasonably accurate decisions without the use of System 2. However, other work by St. B. T. Evans, Barston, and Pollard (1983) also shows that System 1 can be a support mechanism for System 2 with different types of biases resulting from each system (For replication, see Klauer, Musch, \& Naumer, 2000).

To support the findings above, others have delved into the actual reason that System 1 is used to support System 2. The primary limitation of System 2 is that an individual's working memory, that which can be stored in short-term memory for quick retrieval and active processing (Barrett, Tugade, \& Engle, 2004), is typically not available for retrieval at a later point in time. As summarized by Thompson (2009), System 1 processes, such as intuition, allow an individual to free up working memory capacity and therefore process larger amounts of more complex information. In addition, it helps to expedite the temporal aspects of the process to reduce the number of steps necessary to process the information (St. B. T. Evans, 2010). This reduction in steps should reduce the likelihood of computational errors and decrease the cognitive load on the individual thereby avoiding the negative drawbacks presented by bounded rationality (e.g. Simon, 1947).

\subsubsection{A "New" Perspective: Fuzzy Trace Theory}

As shown above, there appears to be mixed support for most of the existing theories of decision making. While the logical and information processing perspectives seem to fall short due to the imperfect nature of human decision making and information recall (e.g. Shannon, 
1987), the intuitive perspective also fails to account for the sequential nature of information processing (Brainerd \& Reyna, 1990). In response to these deficiencies, Brainerd and Reyna (1990) developed fuzzy trace theory to accommodate the shortcomings of traditional approaches. It attempts to incorporate the supported ideas contained within the three prominent mathematical metaphors of decision making to create a more accurate representation of human decision making. Early work addressing the logical and intuitive perspectives provided conflicting evidence with logical scholars suggesting that individuals process information in a manner similar to the way that computers process information, while intuitive scholars assert that decision making is less mechanistic and structured. A recurring empirical finding that spurred the current line of inquiry suggested that individuals can properly reason based on information that they are presented, but they cannot efficiently discriminate between actual information that was presented and the bottom-line meaning of the experience (e.g. Bransford \& Franks, 1971). Fuzzy trace theory attempts to integrate these two perspectives and incorporate conflicting results by describing human cognition as a continuum with the above traditions being opposite poles of the continuum.

Fuzzy trace theory is a dual processing perspective that is based on two recurring themes in the decision making literature. First, theorists propose that both memory and understanding exist in the form of a code that lacks surface details. Second, the theory also suggests that specificity of recall responses depend on situational factors (Brainerd \& Reyna, 2001). The theory posits two levels of specificity with regards to knowledge retention based on the above statements. Verbatim representations are precise and detailed representations that include exact information for use in analytical processes (Brainerd \& Reyna, 1990). On the other hand, gist representations are basic, bottom-line understandings of the meaning of information or 
experience rather than precise details (Brainerd \& Reyna, 1990). The foundational hypothesis of this theory is that individuals encode information with varying levels of precision and ultimately gravitate toward the least precise level that is deemed to be acceptable by the decision maker (Reyna \& Brainerd, 1995b). Despite its existence in the psychology literature for almost 25 years, the organizational literature has yet to embrace the fuzzy trace perspective. The following sections provide a review of the main tenants of fuzzy trace theory that could inform the current and future organizational literature.

Gist Extraction. According to fuzzy trace theory, individuals "mine" detailed information for senses, patterns, and gists that allows them to reduce the information to only its essential meaning (Brainerd \& Kingma, 1984). This is arguably the most important foundational premise of fuzzy trace theory in that individuals reportedly distill specific information down to its basic meaning to simplify cognitive processes (Brainerd \& Reyna, 1990). As noted in other theories of cognitive processing (e.g. cognitive load theory; Sweller, 1988), individuals have a limited amount of active, working knowledge with which to make decisions and solve problems. According to fuzzy trace theory, individuals "mine" detailed information for senses, patterns, and gists that allows them to reduce the information to only its essential meaning (Brainerd \& Kingma, 1984). In addition, individuals may encode gist information at varying levels of abstraction creating a "hierarchy of gist" (Reyna, Brainerd \& Connolly, 1990). Individuals create this hierarchy by determining the amount of precision that they deem to be necessary in future recall. For example, an individual would likely create more specific gist representations when confronted with information regarding a problem at work than when storing information presented in a comedic movie. 
In addition to the above logical examples, gist information processes enjoy empirical support in the existing literature. For example, empirical reviews provided by Reder (1982) and Kintsch (1988) support the notion that information encoding is in fact a gist-driven process. In addition, other evidence addressing latent learning (e.g. Tolman, 1948) and learning sets (e.g Harlow, 1949) provides evidence to suggest that individuals extract information in gist form for future processing. For example, Tolman's (1948) work in latent learning showed that individuals can learn without reinforcement or immediate response by developing a bottom-line meaning for recall at a later point in time. All of this evidence suggests that individuals distill precise information down to its basic meaning for storage and future processing.

Fuzzy to Verbatim Continuum. Another important component of fuzzy trace theory is its assertion that information specificity falls on a continuum from fuzzy traces to verbatim detail. This suggests that information varies along a continuum of exactness, with less exact information at the fuzzy trace end and more exact information at the verbatim detail boundary (Reyna et al., 1990). This assertion proposes a compromise between the earlier theorists in the logical and intuition traditions. On one hand, intuitionists suggested that individuals follow few rules when making decisions which allows new ideas to evolve from the human mind (Heyting, 1959). On the other hand, the logicists proposed that cognition occurs when individuals follow mechanistic rules similar to computer processing (Shannon, 1998). Fuzzy trace theory integrates these perspectives to suggest that human cognition fluctuates along a continuum that is bounded on either end by the intuitionistic and logistic perspectives respectively.

To more accurately describe the above phenomena, fuzzy trace theorists suggest that individuals create a "hierarchy of gist" to organize their stored mental representations (Brainerd \& Reyna, 1988). For example, past research has shown that individuals vary the specificity of the 
information that they store depending on the perceived degree of specificity necessary when providing a response for the given task. In addition, fuzzy trace theorists (e.g. Brainerd \& Reyna, 1988) draw on existing theory for additional support by pointing to evidence suggesting that cognitive processes differ based on situational factors (e.g. Sweller, 1988; Sweller, Chandler, Tierney, \& Cooper, 1990). In all, the existing literature provides fairly clear evidence that individuals alter informational specificity with regards to cognitive processing based on situational factors such as task requirements.

The Fuzzy Processing Preference. As an extension of the above evidence that information processing occurs along a continuum of specificity ranging from gist to verbatim details, the current literature also suggests that individuals generally gravitate toward the gist end of the spectrum when processing information. Reyna and colleagues (1988) suggested that individuals engage in this activity to increase the speed and effectiveness of cognitive processing while also decreasing cognitive load (Sweller, 1988). Fuzzy trace theorists provide several reasons for the existence of this phenomenon: First, theoretical and empirical evidence suggested that richly detailed information is not stable in long-term memory, which makes it less likely to be encoded in the first place and more susceptible to false memory upon retrieval (Bransford \& Franks, 1971). Second, fuzzy traces are more malleable and their fluid structures allow individuals to manipulate stored information to achieve a higher level of understanding (Reyna et al., 1988). Third, processing of verbatim information is much more complex than gist information processing which increases the likelihood of error and processing failure (Reyna et al., 1988). Fourth, parallel processing composed of primarily gist information with only the necessary verbatim details overcomes the limitations of approaches presented by Piaget (1926/1960) and Hilbert (1923) that suggest linear and sequential processes. As shown by Estes 
(1988), it appears that information processing is actually globally parallel with only locally serial aspects when necessary. Fifth, psychological inquiry repeatedly shows that individuals tend to choose the lowest level of cognitive effort necessary when making decisions with gist information normally considered as being sufficient (Reyna et al., 1988). All of this evidence seems to support the notion that individuals gravitate toward the utilization of gist information during cognitive processing.

Although somewhat clear on the surface, additional discussion of the fuzzy processing preference is necessary regarding its relationship to the fuzzy to verbatim continuum. The apparent conflict between the fuzzy processing preference and a verbatim shift for task-related processing appear to be at odds without further explanation. On one hand, Brainerd and Reyna (1988) suggested that individuals have an innate preference to process information that falls on the fuzzy trace end of the spectrum. On the other hand, Reyna and colleagues (1990) propose that task-relevant information is processed closer to the verbatim end of the continuum than information that is not task-relevant. The existing fuzzy trace literature makes little attempt to integrate these components, which could be detrimental to the application of fuzzy trace theory. By integrating these propositions, it appears that individuals still prefer processing toward the fuzzy end of the continuum, but they utilize additional verbatim details when they perceive information to be task-relevant. Figure 1 provides a graphical representation of this proposition by showing the predictions of fuzzy trace theory, intuitionism, and logicism on the fuzzy to verbatim continuum. As shown below, the logical and intuitional perspectives anchor the continuum at the verbatim and gist poles respectively. However, the fuzzy trace perspective lies closest to the gist end of the continuum, with task-related processing occurring closer to the verbatim pole than non-task-related processing. 
Insert Figure 1 About Here

Reconstruction of Short-Term Memory. The literature addressing human memory has long held that short-term memory and long-term memory are useful designations to separate "working space" versus "storage space" in human cognition (e.g. St. B. T. Evans, 2008). In addition, the existing literature has long suggested that information stored in long-term memory can be reconstructed for future processing, while details that are not shifted from short-term to long-term memory are lost in processing (e.g. Craik \& Lockhart, 1972). To complement this assertion, theorists suggested that short-term memory is comprised of a verbatim read out in which individuals retain very small amounts of detailed information (e.g. Atkinson \& Shiffrin, 1968; Miller, 1956). On the other hand, Kolodner (1983) suggested that long-term storage was characterized by the reconstruction of gist meanings from stored information. However, fuzzy trace theorists suggest that we should look at this issue from a slightly different lens. Brainerd and Reyna (1988) assert that the comparison of verbatim information stored in short-term memory and gist information stored in long-term memory is an oversimplification. As a response, they propose the model described in Table 2 which includes reconstruction of verbatim traces and the simple recall of gist information. The quadrants addressing the reconstructive retrieval of gist traces (long-term memory) and the simple retrieval of verbatim traces (shortterm memory) have been addressed extensively in the literature (St. B. T. Evans, 2008) and are not unique to fuzzy trace theory. In addition, the idea that simple retrieval of gist traces is fairly basic given that basic inquiry, such as remembering whether there were more women than men 
on a project team, does not require reconstruction of basic facts and can be answered by simple retrieval of gist information (Brainerd \& Reyna, 1988). The primary contribution of fuzzy trace theory to the memory reconstruction literature can be found in the quadrant addressing reconstructive retrieval of verbatim traces.

Insert Table 2 About Here

The assertion that short-term memory can be reconstructed is based on both theoretical and empirical foundations. In theory, the reconstruction of short-term memory is equated to "working backward" in a math problem. By piecing together precise details from most recent to more tangential, individuals may be able to recall specific details that were not moved to longterm storage (e.g. Brainerd \& Reyna, 1988; Johnson \& Sherman, 1990). For example, an individual might mentally "retrace" his or her steps in an attempt to find car keys that were left in a forgotten location. To demonstrate this principle, LaTour, LaTour and Brainerd (2014) provided empirical evidence of short-term memory reconstruction and also found that individual difference factors, such as need for cognition, can impact an individual's propensity to reconstruct information based on precise details. Other studies have examined verbatim reconstruction directly to show that individuals can reconstruct specific details through sequential recall (e.g. Lakshmanan \& Krishnan, 2009). This assertion is very important to the human resource management literature when addressing employee selection for several reasons. First, the assertion that verbatim details can be reconstructed for future processing suggests that individuals are able to reconstruct specific details about interactions with candidates for future 
recall and decision making. Second, it appears that information gained during brief periods of exposure may ultimately impact future decision making. These propositions are valuable contributions to the organizational literature in terms of the information used when making employment-related decisions.

\subsection{TALENT ACQUISITION AND EMPLOYMENT INTERVIEWS}

When considering the application of decision making theory to an organizational context, there are two primary streams of literature that apply. First, scholars have placed a heavy emphasis on decision making among executives in an attempt to tie executive cognition to organizational success and failure. For example, scholars have studied the impact of cognition on factors such as corporate governance (e.g. Forbes \& Milliken, 1999), top management team decision making (e.g. Wong, Ormiston, \& Tetlock, 2011), executive compensation (e.g. Pepper \& Gore, in press), and executive risk-taking (e.g. Martin, Gomez-Mejia, \& Wiseman, 2013). The second stream of research that applies decision making theory in an organizational context is the employee selection literature. Although other tools are available, employment interviewing has been the focus of the selection literature for over 100 years (Binet, 1911; Macan, 2009; Levashina Hartwell, Morgeson, \& Campion, 2014). This research attention is warranted by the overwhelming support for the notion that employment interviews are the most commonly utilized and most preferred selection method among practitioners (Lievens, Highhouse, \& De Corte, 2005; Topor, Colarelli, \& Han, 2007). The popularity of interviews as a selection method is primarily based on three factors: predictive validity, reliability, and face validity.

The first issue that should be addressed when considering the utility of selection interviews is reliability. Given that reliability is a necessary but not sufficient condition for 
validity (Ghiselli, Campbell, \& Zedeck, 1981), all selection procedures must be stable enough to produce valid results. Interrater reliability, the consistency of scoring between multiple different raters (Huffcutt, Culbertson, \& Weyhrauch, 2013), is very critical to conducting valid and fair interviews. Although influenced by factors such as structure and training, meta-analytic evidence suggests that interviewers can provide consistent information that allows for consistent comparison of candidates that were interviewed by different recruiters (Taylor \& Small, 2002). In addition, the consistency of scoring between interviewers is often considered to be similar to the test-retest and internal consistency reliability estimates for other types of selection methods such as personality inventories and intelligence tests (e.g. Moscoso, 2000; Taylor \& Small, 2002). For these reasons, employment interviews appear to provide the internal consistency necessary to consider the validity of such measures.

The existing literature has extensively explored the predictive validity of selection interviews. Predictive validity is the ability of a selection method to accurately project future behavior. Often the subject of meta-analysis (e.g. Conway, Jako, \& Goodman, 1995; Huffcutt \& Arthur, 1994; McDaniel, Whetzel, Schmidt, \& Maurer, 1994; Wright, Lichtenfels, \& Pursell, 1989), there is strong evidence to suggest that employment interviews can provide valid data with which to make selection decisions. In addition, evidence suggests that employment interviews can provide useful information that would otherwise be captured with metrics such as cognitive ability tests with a reduced likelihood of adverse impact based on both race and gender (Huffcutt \& Roth, 1998; Moscoso, 2000). In addition, the validity of selection interviews can be improved by adding elements such as structure (Weisner \& Cronshaw, 1988), bias-related interviewer training (Latham, Wexley, \& Pursell, 1975), and behaviorally anchored rating scales 
(Maurer, 2002). Later in this manuscript, these elements will be discussed individually to address the intricacies of their impact on employment interview validity.

The final reason for the prominence of employment interviewing is completely unrelated to the instrument's ability to predict future performance. Face validity is an applicant's perception of the fairness of the interview process and the utility of a selection method for making sound selection decisions (Shotland, Alliger, \& Sales, 1998). Given the near universal use of interviews in employee selection, it is taken as a near universal truth that face validity would be severely damaged if interviews were omitted from the selection process (e.g. Lievens, De Corte, \& Brysee, 2003). As noted by Huffcutt and Culbertson (2010, p. 185), “it is rare, even unthinkable, for someone to be hired without some type of interview." In addition, data collected from hiring managers suggests that they fully expect interviews to be an integral part of the selection process (Lievens et al., 2005). For this reason, it is likely that many organizations would still conduct employment interviews even if the results have little bearing on the final selection process to ensure that their reputation as an employer does not suffer as a result of low face validity. To support this notion, it is often suggested that employment interviews are either the only selection method used in many organizations or an initial screening method that must be cleared before entering into a multiple-hurdle selection process (Levashina et al., 2014). In short, it appears that employment interviews are the dominant employee selection method in the past, present, and future. Given that they provide reliable and valid results, the dominance of employment interviews in employee selection may be a positive quality of the selection process. However, as will be discussed shortly, the validity and reliability of employment interviews is often contingent upon conducting interviews via a specific process that displays several standardized features. 


\subsubsection{Interview Structure}

Despite the current support for the validity of employment interviews, some of the earlier research addressing the topic provided mixed results as to the validity of employment interviews (e.g. Griffitts, 1924; Hunter \& Hunter, 1984). In response to this criticism, interview structure was one of the first factors considered for improving the validity and reliability of employment interviews (e.g. Rodger, 1952; Wagner, 1949). To this day, the human resource management literature is still debating the use of structure and the degree to which interviews should be structured to achieve optimal results (Macan, 2009). Interview structure is often defined as the amount of discretion that is afforded to the interviewer when conducting an interview (Huffcutt $\&$ Arthur, 1994). The current debate centers on the type of structure and amount of structure that produces the most reliable and valid results. Traditionally defined, unstructured interviews allow the interviewer to control the direction and content of the interview and occurs in the form of a free flowing conversation (Latham \& Finnegan, 1993). On the other hand, structured interviews are typically defined as an oral questionnaire that contains predefined questions that are asked of every applicant (Gatewood \& Field, 1987). The current literature suggests that the optimal amount of structure falls somewhere between totally structured and totally unstructured, but the optimal amount of structure is not completely clear.

While the above section discusses interview question standardization, it is also important to consider the standardization used when scoring interview responses. Without scoring and scoring standardization, it is difficult if not impossible to compare multiple applicants using the same criteria resulting in less valid measurement. Early methods proposed for standardizing response structure included the use of behavioral expectation scales (BES), behavioral observation scales (BOS) and trait scales (Wiersma \& Latham, 1986). All of these methods 
attempt to give the interviewer a list of behavioral markers that constitute high and low scores for a particular interview question. The most prominent type of scoring structure in the existing literature is the use of behaviorally anchored rating scales (BARS). BARS present the interviewer with descriptions of several behavioral dimensions that signify a certain level of performance for a particular interview question (Jacobs, Kafry, \& Zedeck, 1980). Unlike the literature addressing interview question standardization, the use of response scoring standardization such as BARS is less clear. For example, evidence suggests that there is very little difference in the inter-rater agreement, validity, and accuracy of each different type of rating system and that each system provides its own detrimental side effects (Kingstrom \& Bass, 1981). This conflicting evidence suggests that highly structured and completely unstructured rating scale options both have inherent flaws that could be addressed by a structure that falls somewhere between these two boundaries.

Given that much of the literature is focused around the basic nomenclature of structured versus unstructured interviews, it should come as no surprise that empirical research abounds addressing the validity of both approaches. In addition, this subject has been addressed by several different meta-analyses. Hunter and Hunter's (1984) meta-analysis that questioned the validity and reliability of employment interviews by suggesting that cognitive ability tests are far more valid than employment interviews, which sparked empirical inquiry on this topic. In response, subsequent meta-analyses recognized the subdivision based on structure to determine its impact on interview validity. For example, Wiesner and Cronshaw (1988) discovered that the validity of structured interviews (.62) was twice that of unstructured interviews (.31). In addition, McDaniel, Schmidt, and Hunter (1988) and Marchese and Muchinsky (1993) found that there is a strong relationship between interview structure and validity. However, this stream of research 
was disrupted by approaches such as that of Huffcutt and Arthur (1994) and Campion, Palmer, and Campion (1997) that proposed the need for a further subdivision of interview structure that extends beyond the simple structured versus unstructured typology.

Developing a Nomenclature for Interview Structure. The current debate regarding the optimal amount of interview structure is largely based on the nomenclature developed by Huffcutt and Arthur (1994) that defines degrees of structure rather than the existence or total lack of structure. This nomenclature was developed in response to the meta-analysis performed by Hunter and Hunter (1984) which suggested that mental ability tests were far superior predictors of future job performance than employment interviews. As noted by Huffcutt and Arthur (1994), the original meta-analysis suffered from several shortcomings such as low sample size, lack of consistency in terms of the moderating influence of structure, and lack of evidence regarding how much structure is enough structure. In response, Huffcutt and Arthur's (1994) empirically developed nomenclature classifies interview structure based on interview question standardization and response scoring standardization. This structure answered numerous calls in the literature to explore the scalar nature of interview structure for both interview questions and response scoring (e.g. Daniel \& Valencia, 1991). The Huffcutt and Arthur (1994) nomenclature is displayed in Figure 2 below and each type of structure is detailed in the following sections.

Insert Figure 2 About Here

An important contribution of the Huffcutt and Arthur (1994) nomenclature is its division of standardization into multiple different levels. In the figure shown above, response scoring 
standardization is divided into three different levels ranging from global assessment (1) to evaluation based on pre-established answers (3) with response scoring based on multiple established criteria as a midpoint (2). In addition, question standardization is described on a scale from no constrains on question variety (1) and asking the exact same questions with no opportunity for follow-up (4). The middle points of question standardization are described as limited constraints (3) and precise question standardization with the interviewer having the choice to follow up on answers (3). Huffcutt and Arthur (1994) then empirically derived a four different types of interview structure (displayed in Figure 2) based on existing empirical studies.

First, it is important to note that the typology recognizes the traditional perspectives of completely structured (Structure IV) and completely unstructured (Structure IV). Both of these structures were included in the empirical analysis to see how the polar alternatives already proposed in the existing literature (e.g. Marchese \& Muchinsky, 1993; Weisner \& Cronshaw, 1988) compare to the integrated approaches of Structures II and III. The integrated approaches account for multiple cells within the structure matrix shown in Figure 2 and are generally described as having some use of formal structure (Structure II) and a high level of structure while still allowing some variability (Structure III). A meta-analysis performed by Huffcutt and Arthur (1994) placed existing studies into the cells displayed in Figure 2 to identify the optimal level of structure for employment interviews. Results suggested that validity increases as structure increases with the caveat that the trend asymptotes at Structure III. This evidence suggests that structured questions and scoring are superior to unstructured interviews with the caveat that providing interviewers with some freedom with regards to follow-up questions and interpretation of answers is more advantageous to a highly rigid structure. 
To further complicate this issue, the typology created by Huffcutt and Arthur (1994) was further expanded by Campion and colleagues (1997) to examine content and evaluation issues in employment interviews. Content was examined by exploring factors such as basing questions on a job analysis, asking the same questions of each applicant, controlling ancillary information, and refusing questions from the applicant until the end of the interview. On the other hand, evaluation was comprised of components such as using anchored rating scales, taking notes, using multiple interviewers, and providing interview training. In essence, this typology is an extension of the original Huffcutt and Arthur (1994) typology with content relating to question standardization and evaluation relating to response scoring standardization. Recently, Levashina and colleagues (2014) added several new components of structure to those proposed by Campion and colleagues (1997) and performed a content analysis of the existing literature to explore the types of structure that are most commonly used for interviewing. While none of the new items were highly prominent, the authors discovered that basing interview questions on a job analysis, asking each applicant the same questions, and asking better questions were the most common themes in the content area while rating each question, using anchored rating scales, and interviewer training were used most commonly the evaluation category (Levashina et al., 2014). That being said, the validity of using each component of structure has not been widely explored. In any case, it appears that the use of structured components in employment interviews increases both the validity and reliability of the process.

Types of Structured Interview Questions. When designing structured interview questions, there are two primary choices with regards to the types of questions that are used in the interview. Behavioral interview questions ask applicants to provide an example of past behavior that would be related to the target job (Janz, 1982). An example of this type of question 
might be to "tell me about a time when you had a disagreement with your supervisor and explain how you resolved the situation while still being respectful to your supervisor." This type of interview question is based on the premise that past behavior is a predictor of future behavior (Janz, 1989). The alterative type of interview questions are situational in nature in that the recruiter asks the applicant to describe how he or she would act when placed in a hypothetical setting (Latham, Saari, Pursell, \& Campion, 1980). For this type of question, the interviewer might ask "Imagine you are approached by a disgruntled customer who bought an item that was defective. What do you do to resolve the customer's current conundrum and ensure that they will visit our store in the future?" This type of interview question attempts to gather behavioral intentions and is rooted in the literature addressing goal-setting theory (Latham, 1989).

Given that both situational and behavioral interviews attempt to predict future performance by capturing information in a similar way, it is not surprising that several scholars have compared the validity of the two methods. Pulakos and Schmitt (1995) were two of the first scholars to provide an empirical comparison of structured interview question types when they found that behavioral interview questions were much more predictive of job performance than situational questions based on a sample of higher-level federal investigative agents. In response, Huffcutt, Weekley, Wisener, DeGroot, and Jones (2001) tested the difference between the validity of situational and behavioral interview questions when hiring at lower levels of the organization, but their results did not reach traditionally accepted levels of significance when testing for validity differences. Later, Klehe and Latham (2005) conducted an additional inquiry utilizing MBA students addressing the validity of question types while also accounting for the use of scoring guides for situational questions. Their results suggest that the difference between behavioral $(r=.34)$ and situational $(r=.41)$ question validity was not statistically significant when 
using scoring guides for both question types. Krajewski, Goffin, McCarthy, Rothstein, and Johnson (2006) performed a near replication of the Klehe and Latham (2005) study and obtained opposite effects by showing that behavioral interview questions account for incremental validity over situational questions. This accumulation of evidence seems to suggest that behavioral interview questions might be more valid when hiring for upper level positions in organizations, but the validity difference between behavioral and situational interview questions at lower levels seems to be non-significant.

\subsubsection{Pre-Interview Interactions and Impressions}

It is important to note that an interviewer's final impressions of an applicant are partially based on interactions outside of the interview itself. Interviewers and applicants often have the opportunity to interact informally at career fairs and during rapport building sessions before the interview commences (e.g. Ulrich \& Trumbo, 1965). The effect of these initial interactions on interview judgments has been addressed to a limited extent in the existing literature. This research stream can be separated into two primary channels. The first subdivision addresses objective information that is provided to the interviewer before meeting the applicant. This includes information from resumes, applications, and biographical information provided by the applicant (Posthuma, Morgeson, \& Campion, 2002). The second subdivision of research addresses interpersonal interactions between applicant and interviewer prior to the commencement of the formal interview. Settings such as career fairs and pre-interview rapport building sessions allow for contact between interviewer and applicant before the interview begins (Posthuma et al., 2002). This research stream is of importance to the employee selection literature as individuals are often susceptible to a confirmation bias. Confirmation biases occur when individual seek out information that supports or confirms their original assessment of 
someone or something (Dougherty \& Turban, 1999). This suggests that impressions made early in the selection process could weigh heavily on the final outcome as interviewers seek out supporting information for their initial impressions.

With regards to interpersonal interactions, research addressing the impact of preemployment interactions on final selection decisions seemed to suggest that confirmatory biases do not significantly impact final selection decisions. For example, Kinicki, Lockwood, Hom, and Griffeth (1990) found that impressions formed during the interview accounted for a large portion of the variance in final hiring decisions, but that initial screening interactions were relatively unrelated to final impressions. Later work by Chapman and Zweig (2005) suggested that intuitive judgments based on initial interactions were not based on job-relevant factors. More recently, Barrick, Swider, and Stewart (2010) provided some additional clarity on this topic when they discovered that individuals making better first impressions during rapport building are more likely to receive job offers and higher interview ratings. In addition, a recent review conducted by Levashina and colleagues (2014) calls for additional work addressing the impact of rapport building on employment decision making and structured interview validity. In summary, the literature addressing the effect of pre-interview interpersonal interactions on final ratings of employability provides conflicting results that do not yet provide clear recommendations for the usage and potential control of rapport building in employment interviews.

Despite the ambiguity present in the literature addressing the impact of pre-interview interpersonal interactions on final ratings of employability, empirical research addressing the impact of objective qualifications provided before the interview is clearer. Macan and Dipboye (1990) provided early evidence to suggest that knowledge of coursework and qualifications provided to the interviewer prior to the interview was highly related to final ratings of 
employability. Later, the same authors proposed that note-taking could potentially reduce the effect of pre-interview impressions, but their results did not support this hypothesis and suggest that pre-interview knowledge does in fact impact final impressions (Macan \& Dipboye, 1994). Additional work performed by Dalessio and Silverhart (1994) also supports this line of research by suggesting that interviewers integrate information obtained from biodata testing with their interview impressions to more accurately predict 12-month survival.

In summary, the current literature addressing the impact of initial interactions on final ratings of employability provides mixed results as to the nature and extent of the effect. However, it is important to note that currently published results are limited in that they often do not capture the entire process of employee selection (e.g. Levashina et al., 2014). For example, much of the current literature often ignores the fact that interviewers and applicants typically meet informally (e.g. career fair interactions) prior to the interview. This initial interaction could form impressions that ultimately impact final ratings of employability. In addition, a limited amount of research addresses the combined effects of both objective information (biodata, cover letters, etc.) and interpersonal interaction on interview outcomes (Levashina et al., 2014). By addressing these issues, future research could help to identify the sources of variance in final ratings of employability and attempt to reduce the potential biases inherent in the interview process.

\subsubsection{Integration with the Decision Making Literature}

As a process, employee selection is simply decision making within a specific context. Given that there is a well-developed literature addressing employee selection, it is not surprising that there is some overlap between the two topics. More specifically, the employee selection 
literature has integrated findings from the general decision making literature to help understand how and why interviewers render judgments (Posthuma et al., 2002). Factors such as decision making heuristics (e.g. Kataoka, Latham, \& Whyte, 1997), order and contrast effects (e.g. Schmitt, 1976), and differential weighting of information (e.g. Harris, 1989) are some of the main junctures at which the mainstream decision making and employee selection literatures intersect.

Anchoring heuristics are often discussed in the existing literature and address an individual's unconscious reliance on initial information that is later adjusted as interpersonal interactions progress (Tversky \& Kahneman, 1974). However, the adjustments made during original impressions is often biased in the direction of the initial impression (e.g. lower initial impressions that are subsequently adjusted upward will ultimately be biased downward due to anchoring) (Bazerman, 1990). This effect was originally established in prospect theory (Kahneman \& Tversky, 1979) and has been explored in the context of accounting-related judgments (Butler, 1986), motivation-related judgments (Cervone \& Peake, 1986), and determining listings for home prices (Northcraft \& Neale, 1987). Empirical evidence addressing this construct suggests that interviewers develop an initial impression of an applicant and modify that impression based on subsequent interactions (e.g. Kataoka et al., 1997). Specifically, Kataoka and colleagues (1997) found that the detrimental effects of anchoring were significantly lessened when employing situational interview questions as opposed to behavioral questions. This form of integration shows the ability of the general decision making literature to help identify the mechanisms through which interviewers make decisions rather than solely focusing on outcomes. 
Another area in which these literatures overlap is through order and contrast effects. This effect suggests that individuals place differential weights on information that they receive based on the order in which it is presented as well as the consistency of the information (Anderson, 1960). Early research addressing this topic in the general decision making literature suggested that information presented early during an interaction is more likely to influence decision making than information presented later in the interaction (Anderson, 1960). The anchors discussed above are posited to form via ordering effects in that the earliest information received by an individual helps to form the anchor (Schmitt, 1976). When integrating this effect into the employee selection literature, scholars focused on the impact of information presented during pre-interview interactions or early in the interview as compared to information presented later in the interview. For example, Arvey and Campion (1982) conducted a review of the empirical literature and suggested that contrast effects and primacy-recency effects influence both applicant ratings and the amount of time that interviewers take when making decisions. Later, Gatewood, Lahif, Deter, and Hargrove (1989) explored the impact of interviewer training on the decision making process and found that training might minimize several factors (e.g. more talking, asking more questions, etc.) that lead to order and contrast effect. This seems to suggest that ordering of information in employment interviews makes a significant impact on the outcome of the interview, but factor such as training could be used to reduce this effect.

Aside from the above, the primary area of overlap between the decision making and employee selection literatures is in the area of biases and stereotypes. It is important to note this this literature is quite vast and a review of its entire contents falls outside the scope of this manuscript. Some examples of the primary topics included in the bias and stereotype literature include similarity biases, confirmatory biases, and stereotypes. Similarity biases were originally 
conceptualized as personality and other deep-level differences in the decision making literature (Byrne, 1971) based on learning theory, but have been replicated and extended in the selection literature to address factors such as personality similarity (e.g. Dalessio \& Imada, 1984) racial similarity (e.g. Lin, Dobbins, \& Farh, 1992), age similarity (e.g. Lin et al., 1992), and gender similarity (e.g. Graves \& Powell, 1995). Results in this area provide mixed support for a similarto-me effect, which suggests that interviewers may favor candidates with qualities similar to their own depending on situational circumstances. Another line of inquiry has addressed the impact of stereotypes, or preconceived notions based on an applicant's membership in a particular group (e.g. gender, race, etc.), on both the applicant and interviewer throughout the process (Klimoski \& London, 1974). Empirical evidence suggest that stereotypes have a significant impact on interview outcomes (e.g. Morgeson, Reider, Campion and Bull, 2008), providing further evidence that information unrelated to job performance impact the selection process. As shown above, the integration of the decision making literature addressing biases and stereotypes has aided in the development of employee selection strategies that reduce the impact of such factors.

The preceding literature review shows that the employee selection literature has in fact incorporated quite a few concepts that were developed in the general decision making literature. However, there are other topics that seem ripe for inclusion in an employee selection context. For example, there are numerous theories in the decision making literature that address the mechanisms behind decision making that could potentially address bias and inaccuracy. Dual processing theories, such as fuzzy trace theory (Brainerd \& Reyna, 1989) and cognitive fit theory (Vessey, 1991), that address information storage, working memory, information recall, and problem solving could provide insights as to the mechanisms that lead to bias and inaccuracy 
rather than simply focusing on the outcome of the process. Such information could potentially improve both the validity and reliability of current selection methods.

\subsubsection{Applicant Factors Influencing the Selection Process}

In a perfect world, the only factors that should impact the outcome of employee selection process are differences between applicants that affect work performance. However, as shown above, that may not always be the case. Individual difference and applicant behavioral factors such as appearance (Riggio \& Throckmorton, 1988), impression management (e.g. Gilmore \& Ferris, 1989), applicant fit (e.g Rynes \& Gerhart, 1990), and personality (e.g. Cortina, Goldstein, Payne, Davidson, \& Gilliland, 2000), are key factors that account for important variance in the outcomes of employment interviews. Each of these factors contributes to final variance in employment interview outcomes, but their relatedness to future performance is not always clear.

For many practitioners, judging the fit between the applicant and the organization is the primary reason for conducting employment interviews (Rynes \& Gerhart, 1990). Fit is most commonly conceptualized as fit between the applicant and the occupation, job, and organization. Person-organization fit is discussed most commonly in the selection literature and is conceptualized as the match between an employee and the organization that occurs when both parties' needs are met or they share fundamental characteristics (Kristof, 1996). The existing literature suggests that interviewers provide higher recommendations for applicants that are deemed to "fit" with the organization's ideals and values (e.g. Cable \& Judge, 1997). In addition, other research shows that applicants that interviewers deem to be similar to themselves in terms of gender (Graves \& Powell, 1995), personality (Schneider, Smith, Taylor, \& Fleenor, 1998), 
and other individual differences are rated as being a better "fit" with the organization. Metaanalytic evidence supports this line of inquiry by suggesting that perceived fit during preemployment activities is strongly related to job satisfaction, organizational commitment, and turnover intentions (Kristof-Brown, Zimmerman, \& Johnson, 2005). Although this might be construed as bias in some situations, more recent studies suggest that the relationship between similarity and hiring recommendations is mediated by factors such as projected performance rather than liking (e.g. Garcia, Posthuma, \& Colella, 2008). Applicants also assess fit through the selection process by gathering information from the interviewer regarding the organization's culture. Research addressing realistic job previews, detailed representations of normal work activities and procedures provided to the applicant, suggests that applicants can remove themselves from the process when they foresee poor fit leading to increases in retention and providing more accurate initial expectations (Phillips, 1998). Holistically, this literature seems to suggest that person-job fit and person-organization fit are crucial factors that impact both the outcome of the selection process as well as the ultimate success of future employees.

Another applicant factor that is a strong predictor of interview outcome is the applicant's personality. Most research addressing personality is based around the Five Factor Model of personality which utilizes five trait clusters to describe personality: openness to experience, conscientiousness, extroversion, agreeableness, and neuroticism (Goldberg, 1992). In addition to personality similarity as discussed above, personality factors such as extraversion, conscientiousness, and need for achievement have been shown to modestly relate to interview outcomes (Posthuma et al., 2002). Additionally, these personality factors have been ultimately linked to future job performance (e.g. Thoresen, Bradley, Bliese, \& Thoresen, 2004), providing additional evidence for the validity of employment interviews. While personality does appear to 
be captured within employment interviews, debate still exists in the literature as to the need for additional personality testing beyond the interview (e.g. Macan, 2009). In any case, it is clear that applicant personality makes a substantive impact on the outcome of employment interviews.

While other applicant characteristics such as appearance also impact interview outcomes, the final applicant factor to be addressed in this manuscript is impression management. Impression management is the process of influencing the images that others form during social interactions (Schlenker, 1980). Given the interpersonal nature of employment interviews and the importance of interviewer impressions, impression management is likely to be employed by applicants to increase the likelihood of a positive outcome. Recent meta-analyses show that impression management makes a significant impact on interview outcomes, but that those effects can be suppressed through the use of structured interviews (Barrick, Shaffer, \& DeGrassi, 2009). In addition, individuals vary their usage of impression management depending on whether questions are behavioral or situational in nature (Levashina et al., 2014). While the debate surrounding the prevalence of usage and the ultimate impact of impression management in employment interviews continues, it appears that it may be more difficult to fake during structured employment interviews as opposed to other types of selection procedures such as personality tests (Levashina et al., 2014).

In sum, the literature addressing employee selection is well developed and has clarified the primary usages and outcomes of interview processes. As a result, practitioners now have better recommendations regarding interview structure, question types, decision making processes, and the applicant qualities that are best assessed using employment interviews. However, as shown during discussions of integration with the decision making literature, the employment interview literature could benefit from integration with other decision making 
perspectives. Doing so would allow for further exploration of the decision making mechanisms contained within employment interviews.

\subsection{THIN SLICES OF BEHAVIOR: LEARNING A LOT FROM A LITTLE}

In the existing employee selection literature, it is widely implied that employment interviews do and should last between thirty minutes and one hour to acquire sufficient information for selection decisions (e.g. Tullar, Mullins, \& Caldwell, 1979). However, another perspective on information gathering in social interactions suggests that it may take only a fraction of that time horizon to collect adequate information for decision making. The thin slicing perspective proposed by Ambady and Rosenthal (1992) suggests that only 5 to 300 seconds are necessary for drawing inferences about another individual. Thin slices of behavior are defined as "short excerpts of social behavior from which perceivers can draw inferences about states, traits, and other relevant characteristics" (Ambady \& Rosenthal, 1992; p. 257). Although earlier work had addressed the utility of brief observations of behavior (e.g. Allport, 1937; Mintz \& Luborsky, 1971; Stiff, Miller, Sleight et al., 1989), Ambady and Rosenthal (1992) consolidated the literature via meta-analysis and provided a theoretical framework on which to base future research. The development of the literature addressing thin slices of behavior is summarized below followed by an empirical review of the studies that followed Ambady and Rosenthal's (1992) meta-analysis.

The work addressing thin sliced of behavior is rooted in the decision making literature addressing System 1 versus System 2 processing. In essence, Ambady and Rosenthal (1992) suggest that System 1 has the ability of producing analytical output that can be used for assessing 
another individual. Theoretically, this perspective extends the earlier work in decision making to suggest that System 1 cognition can produce accurate judgments based on only short periods of exposure (Ambady, 2010). In addition, empirical evidence also suggests that judgments based on thin slices are both intuitive and automatic processes (e.g. Logan, 1992; Wegner \& Bargh, 1998; Ambady, 2010). More specifically, empirical work addressing intuitive processing suggests that time is a critical factor in transitioning from System 1 to System 2 processing (e.g. Stanovich, 1999) with intuitive judgments taking less time than the analytical evaluations produced by System 2. Ambady and Gray (2002) built upon this foundation in finding that that mood impacts the effectiveness of thin slice judgments with sad participants utilizing more deliberate and less effective cognitive processes. In addition, Ambady (2010) provided evidence suggesting that thin slice judgments are still effective even when attention and active cognitive processing are diverted away from the outcome of interest. Earlier work conducted by Vallacher and Wegner (1987) laid the foundation for this research by suggesting that focusing attention and cognitive resources on intuitive and automatic processes decreases processing effectiveness. In short, this accumulation of research suggests that decisions based on thin slices of behavior are intuitive in nature and that attempts to focus attention during intuitive processing could be detrimental to decision quality.

Early research addressing small excerpts of expressive behavior was primarily contained within the marriage and relationship literature. In his foundational study, Gottman (1979), a marital expert, provided evidence to suggest that experts can predict future outcomes with exceptional accuracy based on only brief social interactions. In addition, his work suggests that a thin slice taken at the beginning of the interaction is more useful for predicting outcomes. Future longitudinal work by Gottman and colleagues showed that individuals viewing the first 3 
minutes of a conflict between spouses could accurately predict marital outcomes over a 6 year period (Carrère \& Gottman, 1999). Future work in other fields extended the original findings of Gottman and colleagues by assessing the effectiveness of thin slices in identifying other important outcomes. For example, additional work assessing the effectiveness of thin slicing for predicting teacher effectiveness (Ambady \& Rosenthal, 1993), psychological disorders (Friedman, Oltmanns, \& Turkheimer, 2007), and propensity for violence (Stillman, Maner, \& Beaumeister, 2010) all support the validity of thin slices in decision making.

In their foundational article, Ambady and Rosenthal (1992) performed a meta-analysis using past research that was germane to the thin slicing construct. Given the age of this metaanalysis and the fact that specific research addressing the thin slicing perspective followed the meta-analysis, it is useful to consider the recent literature addressing thin slices of behavior. An empirical review was conducted to summarize the empirical articles that have addressed thin slicing that were published after the original meta-analysis. Primary studies analyzing the validity of the thin slicing perspective were located using the PsychInfo and Google Scholar databases. Search terms included "'thin slices” AND behavior', “thin slicing” AND behavior', “"thin slice” AND behavior.' In addition, a reference search was conducted to explore each article that cited the original theory and meta-analysis conducted by Ambady and Rosenthal (1992). This search resulted in 21 articles that contained 30 studies. Table 3 shows the results of this review and details the factor being assessed using thin slices of behavior, moderators used to identify boundary conditions, referent comparison (long-term versus random chance), slice length, and results. The results will be discussed in further detail in the following sections. 
Insert Table 3 About Here

\subsubsection{Accuracy of Thin Slices}

When considering the utility of thin slices, the validity of the construct is solely measured by its predictive power. In the existing literature, two different referents are used to assess the validity of the thin slicing perspective: random chance and thicker slices. Studies comparing thin slicing to random chance simply assess whether or not the thin slicing perspective provides useful information for making decisions. In the original meta-analysis, Ambady and Rosenthal (1992) used results from 38 studies to suggest that the use of thin slices of behavior to predict outcomes provides significantly better results than random chance $(\bar{x}=.41 ; \tilde{x}=.30 ; \mathrm{p}<.001)$ with every study in the sample reporting significant results. In addition, 21 studies have been conducted since 1993 utilizing thin slices ranging from 2 seconds to 2.5 minutes to assess the utility of thin slices of behavior as compared to random chance. Every study within the empirical review also reports significant results. These combined results provide overwhelming support for the thin slicing perspective and its utility for assessing the behaviors and attitudes of others.

Although less plentiful, existing research has also explored the utility of thin slices by assessing the information gained during thin followed by thicker slices of observation. In each study, the authors expose participants to a focal actor for a short window of time, collect data addressing their perceptions of the focal actor, expose participants to the focal actor for a longer period of time (a thick slice greater than 5 minutes), and then ask participants to assess their perceptions of the focal actor for a final time. Ambady and Rosenthal (1993) provided one of the 
first empirical tests comparing thinner to thicker slices of behavior when they found that there was not a significant change in teacher effectiveness ratings when comparing thin slices of 6,15 , and 30 seconds to periods of observation greater than 5 minutes. Additional work provided similar conclusions when comparing thin slices to thicker slices when assessing personality and intelligence (Borkenau, Mauer, Riemann, Spinath, \& Angleitner, 2004), coding of behavior (Murphy, 2005), and negotiation outcomes (Curhan \& Pentland, 2007). However, Tom, Tong, and Hesse (2010) provide mixed support for the thin slicing perspective by showing that thin slices of behavior have predictive utility when assessing teacher evaluations, but new information is gained when moving from thin slices to thicker slices of behavior. To further complicate the issue, Tom and colleagues' (2010) study used thin slices of 30 seconds with the other studies using varied slice lengths ranging from 6 seconds (Ambady \& Rosenthal, 1993) to 5 minutes (Curhan \& Pentland, 2007) suggesting that their use of a 30 second slice of behavior was not the cause of the conflicting results. In all, there appears to be support for the notion that thick slices of behavior do not provide significantly more information than thin slices of observation. However, recent conflicting results suggest that additional empirical testing may be necessary to further address this phenomenon.

\subsubsection{Effectiveness for Predicting Different Traits}

Despite the general support for the thin slicing construct, research also suggests that different types of behavior may require varying slice lengths for adequate identification (e.g. Carney, Colvin, \& Hall, 2007; Borkenau, Brecke, Mottig, \& Paelecke, 2009) . In other words, it may take an individual longer to accurately predict conscientiousness based on thin sliced than when assessing openness to experience. Much of this research has addressed the slice length necessary for obtaining accurate perceptions of a focal actor's personality. In their original meta- 
analysis, Ambady and Rosenthal (1992) used 38 studies to show that there is no correlational increase when moving from 30 second slices to 300 second slices. However, recent research has taken a more fine grained approach to assess the impact of exposure length on the accuracy of perceptions.

As an illustration of the above concept, Carney and colleagues (2007) assessed the utility of five different slice lengths, 5, 20, 45, 60, and 300 seconds, to assess the "Big Five" personality traits, positive and negative affect, and intelligence. Results suggested that negative affect, extroversion, conscientiousness, and intelligence were judged moderately well after an exposure period of only 5 seconds. On the other hand, positive affect, neuroticism, openness, and agreeableness required longer periods of exposure to achieve similar levels of accuracy. That being said, all of the above characteristics achieved desired levels of accuracy when using Funder's (2001) realistic accuracy model after an exposure length of 300 seconds. In addition to the above, Borkenau and colleagues (2009) further explored the impact of slice length to show that extroversion can be accurately assessed after an exposure period of only 50 milliseconds. This accumulation of evidence seems to suggest that shorter terms of exposure (e.g. 30 seconds or less) may be adequate for accurate prediction in some situations, but that slice lengths nearing the upper boundary (e.g. 300 seconds) of thin slices appear to be useful across constructs. The practical implication of this finding is that the type of individual difference being assessed might be important when choosing a very short slice length, but less important when choosing a longer period of exposure that is still classified as a thin slice (e.g. between 45 and 300 seconds).

\subsubsection{Thin Slicing in an Organizational Context}


Although limited, the thin slicing perspective has been integrated and tested in an organizational context. For example, Kaul and Schimdt (1971) provided some of the earliest work addressing thin slices in an organizational context when they found that thin slices were useful for assessing interviewer trustworthiness. Later, Blanck, Rosenthal, Vannicelli, and Lee (1986) tested thin slicing in a performance management context by displaying the utility of thin slices for supervisor performance ratings. More recently, Ambady and colleagues (2006) provided evidence to suggest that salesperson effectiveness can be predicted via thin slices and Curhan and Pentland (2007) found that thin slices from the beginning of a negotiation can predict the final negotiated outcome. Each of these studies shows that thin slices of behavior can be useful for obtaining information used to make organizational decisions. However, there are obviously many more organizational applications that could be considered such as employee selection, executive decision making, and partnering decisions. This future research could help to uncover the processes behind decision making, the stages of decision making in which bias occurs, and the potential benefit, harm or non-significance of thicker slices of behavior.

As an extension of this existing research, the thin slicing perspective could be very useful for informing an ongoing debate in the employee selection literature. Current research suggests that recruiters often resist selection decision aids in favor of more subjective and intuitive judgment (e.g. Rynes, Colbert, \& Brown, 2002; Lievens et al., 2005). When addressing this phenomenon, scholars are currently split as to whether this reliance on intuitive judgments is a benefit (e.g. Ambady, 2010) or a hindrance (e.g. Highhouse, 2008) to effective employee selection. By integrating these streams of research, the thin slicing perspective seems to suggest that intuition could provide useful information for making employment-related decisions.

\subsection{COMPENSATION AND PAY FOR PERFORMANCE}


In the United States, labor costs for organizations total just over $70 \%$ of the total costs in the economy (U.S. Bureau of Labor Statistics, 2014). In addition, many organizations' total expenditures are dominated by labor costs. For these reasons, it is not surprising that employee compensation has been studied for hundreds of years (e.g. Smith, 1776/1976). Economists often focus on the distribution of wages between organizations and throughout the economy while psychologists typically study the impact of compensation on the individual via performance, satisfaction, and retention among many others (Gerhart \& Rynes, 2004). Given the goals of this manuscript, the psychological impact of compensation will be the primary focus of this review. Both organizational leaders and researchers have long been interested in the motivational potential of employee compensation. While organizational leaders aim to get the most "bang for their buck" when paying employees, researchers have often focused on the potential positive (e.g. Vroom, 1964; Locke, 1968) and negative (e.g. Deci \& Ryan, 1985) ramifications of compensation decisions. In any case, employee compensation is an important topic in both research and practice that consumes a large portion of organizational budgets and can potentially serve as motivation for employees.

\subsubsection{Economic Perspectives}

Early work addressing employee compensation was dominated by economic research (Gerhart \& Rynes, 2004). Classical economist Adam Smith (1776/1976) was an early contributor to this discipline when he noted that trades that are more difficult to learn are compensated at a higher rate than easier trades. He also suggested that individuals seek to maximize their total utility and therefore seek out the highest possible compensation for their skills and effort. This

perspective is easily displayed on a supply and demand curve with an employee's compensation level being located and the intersection of the supply and demand curves. However, later 
economic work addressed the fact that markets are not completely efficient and therefore employee compensation may deviate from equilibrium due to market and external factors. Due to these factors, institutional economists modified the neoclassical perspective to suggest that institutional forces, such as unions, equity beliefs, and ability to pay, impact pay administration and drive the differences in compensation across the economy (e.g. Commons, 1934). However, this perspective suffered from its own faults in that it moved away from earlier focus on market factors and was not strongly supported by empirical evidence (e.g. Segal, 1986). To address some of these issues, post-institutionalists such as Kerr and Dunlap proposed a balance between institutional and market forces when explaining variance in compensation levels. This perspective suggests that market factors drive wages toward equilibrium levels while institutional factors cause wages to be "sticky" (Gerhart \& Rynes, 2004). For example, Kerr (1954) highlighted the imperfect nature of labor mobility by displaying the transition costs of changing jobs. In addition, organizations also create their own internal labor markets that impact compensation levels throughout the organization (Doeringer \& Piore, 1971). In short, market factors seem to be the primary drivers of employee compensation, but institutional factors also impact compensation levels (Coase, 1998). Despite the purely economic nature of this research, there are also psychological ramifications that result. For example, market factors may be the driver of compensation and labor movement, but institutional factors such as ability to pay and organizational strategy can limit the ability of organizations to attract and retain talent given the "sticky" nature of employment and wages. This type of extension provided the early foundations of psychological research addressing employee compensation.

\subsubsection{Psychological Perspectives - Can Compensation Motivate?}


From its earliest beginnings, the field of psychology has focused on motivation when addressing employee compensation. Despite a lack of empirical support, early work addressing the psychological effects of compensation laid the foundation for more modern and empirically supported theories. One of the earliest psychological theories applied to compensation was formulated by Maslow (1943), which suggested that compensation may not be motivational at all. His original hierarchy suggested that individuals have an innate hierarchy to fulfill needs in a particular order starting with physiological and safety (e.g. food, housing, and sleep), and moving up the hierarchy to love (e.g. belongingness), esteem (e.g. achievement and competence) and self-actualization (being all that I am ever capable of being). Maslow (1943) posited that compensation helped individuals meet their most basic physiological and safety needs and therefore were much less motivational than other aspects such as work engagement and identity. Later, Herzberg (1959) also questioned the motivational nature of compensation when he proposed his two-factor theory. Two-factor theory suggests that job satisfaction is driven by motivational (factors of the work itself that contribute to achievement and psychological growth) and hygienic (basic needs driven by one's "animal nature”) factors (Herzberg, 1959). Herzberg's primary assertion regarding compensation is that money is more likely to serve as a hygienic factor that has the potential to reduce dissatisfaction, but lacks the ability to improve satisfaction (Gerhart \& Rynes, 2004). This perspective suggests that compensation has the ability to suppress negative reactions in the workplace, but fails to produce positive reactions. Although these theories are largely unsupported by empirical research (e.g. Miner, 1980), they have positively impacted the field by contributing to the development of more modern perspectives such as job characteristics theory (JCT; Hackman \& Oldham, 1976). These modern perspectives attempt to improve job design and thereby appeal to higher order needs of individual employees. 
Cognitive Evaluation Theory. Despite the lack of support for both Maslow and Herzberg's theories, cognitive evaluation theory (CET; Deci and Ryan, 1985) provides opposition for the motivational nature of compensation and enjoys more empirical support than earlier perspectives. The primary principles of CET are based on the original ideas of Lepper, Greene, and Nisbett (1973), who suggested that extrinsically motivated behaviors create a psychological tie between extrinsic motivators and their behavior, which reduces the individual's interest in the behavior. CET's primary premise is that workplace motivation should be subdivided into two categories: intrinsic and extrinsic motivation. Extrinsic motivation is a result of factors outside of the individual such as compensation and benefits. On the other hand, intrinsic motivation is produced inside of the individual and is based on an individual's need for competence and self-determination (Deci \& Ryan, 1985). This over justification effect simply suggests that increasing the prominence of extrinsic motivators can have a detrimental effect on intrinsic motivation as a result of a focus shift from the enjoyable aspects of work to the financial outcomes of effort (Gerhart \& Rynes, 2004). In addition, work by Ryan, Mims, and Koestner (1983) suggests that the over justification effect can be avoided if individuals do not perceive compensation to be behaviorally controlling.

Most researchers at the time accepted the propositions contained within CET given its logical internal consistency as well as early empirical support (e.g. Lepper et al., 1973). However, later work called into question the utility of CET. For example, Eisenberger and Cameron (1996) performed a meta-analysis to test for a detrimental effect of extrinsic compensation on intrinsic motivation. Using 83 studies, they found that reward condition did not have a significant impact on either free time taken by the participant or overall satisfaction. In response, Deci, Koestner, and Ryan (1999) performed a meta-analysis to further explore the 
relationship. While the results did provide more support for the detrimental effect of extrinsic motivation on intrinsic motivation as compared to the Eisenberger and Cameron (1996) study, Deci and colleagues (1999) also found that performance-contingent rewards did not significantly impact intrinsic motivation. In total, this accumulation of evidence seems to suggest that there may be a motivational component to compensation. In support of this assertion, there are several theories that explore the motivational benefits of compensation and enjoy a considerable amount of empirical support.

\subsubsection{Psychological Perspectives - Incentive Compensation}

Despite early theories suggesting the contrary, case examples abound that would support the motivational characteristics of compensations and the outcomes that it can produce (e.g. Kerr, 1975). For example, the classic case used to show the motivational effects of compensation is the case of Lincoln Electric. As compared to its competitors, Lincoln Electric's assemblers showed dramatic increases in both output and quality in response to a modification in their pay levels and structure to include incentive compensation (Gerhart \& Rynes, 2004). In addition, absenteeism and turnover are dramatically lower at Lincoln Electric as compared to their competitors. To complement this evidence from individual cases, motivational theories such as reinforcement theory (Thorndike, 1905; Skinner, 1938), expectancy theory (Vroom, 1964), equity theory (Adams, 1965), and goal-setting theory (Locke, 1968) also inform this conversation.

Reinforcement Theory. One of the earliest perspectives addressing the motivational impact of compensation was proposed by Skinner (1938) by expounding on earlier work from Thorndike (1905). Reinforcement theory, also termed operant conditioning by Skinner (1937), 
suggests that behavior is a function of its consequences and can be controlled via external reinforcement techniques (e.g. Staddon \& Cerutti, 2003). Behavior manipulation is implemented by creating a reinforcement schedule that delivers a reinforcer according to a well-defined rule (Stadddon \& Cerutti, 2003). While many early studies in this area were conducted using animals (e.g. Skinner, 1938), later work completed using human subjects is more applicable to an organizational context. The primary foci of this theory are the sign of reinforcers (positive vs. negative reinforcement), temporal sequencing (continuous vs. intermittent), and outcome stability (fixed vs. variable).

The original focus of reinforcement theory addressed the ability of both positive (providing benefits for displaying productive behavior) and negative (instituting punishment for displaying counterproductive behavior) as motivators of human behavior (Staddon \& Cerutti, 2003). Recent research has shown that positive reinforcers may be better motivators of human behavior given that they are less likely to induce a counterproductive response and less likely to be deemed as controlling by the recipient (Deci \& Ryan, 2010). When considering continuous and intermittent distribution schedules, research suggests that situational factors influence the utility of each method. For example, intermittent distribution might be better in a sales context in which employees are given positive reinforcement for selling an item at the time of the sale (e.g. Barling \& Beattie, 1983). On the other hand, managerial employees may show more improvement as a result of continuous distributions such as annual bonuses for meeting financial goals in that these rewards address long-term performance and address the principal-agent problem (Lambert, Larcker, \& Weigelt, 1993).

Finally, the stability of the outcome appears to impact the motivational utility of compensation. This is typically manipulated in an organizational context by choosing either a 
fixed incentive value for reaching a specified level of performance or instituting a stepped incentive system for increasingly higher levels of performance. Recent studies have shown that tiered systems typically result in higher performance for production workers (e.g. Bateman \& Ludwig, 2004), but less evidence exists surrounding managerial and non-production employees. In all, reinforcement theory may not appear regularly in recent journal publications, but it plays a major role as a foundation for subsequent theorizing in employee motivation. That being said, the primary limitation of this theory was identified by Deci and Ryan (2010), who point out the potentially negative effects of incentive systems when they are deemed to be overly controlling. Subsequent theories address this issue and place added emphasis on the individual's perceptions of incentives rather than objective incentive levels.

Expectancy Theory. One of the most widely used theories for explaining the relationship between compensation and performance is Vroom's (1964) expectancy theory. In his theory, Vroom proposes that employee performance is a function of two factors: ability and motivation (Vroom, 1964). It is suggested that ability is a necessary, but not sufficient condition for performance with motivation being the intervening factor that allows employees to use their abilities in the workplace. This process is suggested to occur in three separate stages that are displayed in Figure 3 below. As shown below, Vroom's (1964) theory predicts that three relationships are crucial to predicting the motivational impact of rewards system: effortperformance, performance-rewards, and rewards-personal goals. His primary assertion is that these three relationships have a multiplicative effect that account for the total "motivational force” of employee rewards (Vroom, 1964; Gerhart \& Rynes, 2004). 
Insert Figure 3 About Here

When looking at the total model presented above, expectancy theory suggests that individuals must feel as though their effort will lead to high performance in the workplace (expectancy), their performance will be recognized through organizational rewards (instrumentality), and that those organizational rewards will be of value to the individual (valence). In the existing literature, expectancy theory has largely been used to understand the effects of moving from one type of pay system to another (Gerhart \& Rynes, 2004). For example, Schwab (1973) discovered that group reward systems may not provide the same motivational impact as individual systems given that the expectancy component of the theory may be reduced. In addition, other work has address the shift from an objective measure of performance to subjective measures when distributing incentives. Overall, research in this area has suggested that situational factors such as organizational level (e.g. Pearce, Stevenson, \& Perry, 1985) and the relationship of objective measures to overall employee performance (e.g. Bebchuk \& Fried, 2004) impact the effectiveness of varying levels of objectivity in incentive distribution, while a smaller group of scholars suggest that a mix of objective and subjective measures might provide the best outcome (e.g. Baker, Gibbons, \& Murphy, 1994).

In terms of model testing, the individual components of expectancy theory are largely supported by empirical research. For example, Van Eerde and Thierry (1996) conducted a metaanalysis suggesting that valence (.21), instrumentality (.16), and expectancy (.22) are all significant predictors of employee performance. However, the multiplicative effect, termed "motivational force" by Vroom (1964), was not supported by the meta-analysis as the valence, 
instrumentality, and expectancy components did not significantly interact to predict additional variance in performance. That being said, questions regarding the reliability of moderation testing in older meta-analyses (e.g. Cortina, 2003) as well as Van Eerde and Thierry's (1996) own questioning of suboptimal data used in many studies included in their meta-analysis complicate the issue. Several more recent studies using primary data, meta-analytic techniques, and theoretical integration have shown support for expectancy theory in the context of training motivation (Colquitt, LePine, \& Noe, 2000), perceptions of politics (Brouer, Harris, and Kacmar, 2011), and work stress (Meurs \& Perrewé, 2011). In short, there appears to be clear support for the motivational benefits of the individual components of expectancy theory (valence, instrumentality, and expectancy), but the current literature has failed to reach a consensus with regards to the multiplicative "motivational force" effect.

Equity Theory. Another motivational perspective regarding employee compensation was presented by Adams (1965) in the form of his equity theory. Equity theory states that individuals desire to achieve a state in which their inputs in the workplace are equal to the outcomes that they receive (Adams 1965). In colloquial terms, employees tend to determine "how much buck they get for their bang." Within this paradigm, individuals are less concerned with absolute levels of outcomes (e.g. compensation), but rather they focus on changes in compensation and differences between their own compensation. As a construct, this effect is typically termed distributive justice (Adams, 1965).

The literature addressing distributive justice is well-developed and results are quite consistent. Empirical evidence supports the salient nature of compensation changes (even in cases of incentive compensation; Brown \& Huber, 1992) and the detrimental effect of social comparisons in which one perceives underpayment inequity (e.g. Folger \& Konovsky 1989; 
McFarlin \& Sweeney, 1992; Choi \& Chen, 2007). In addition, meta-analytic evidence has tied distributive justice to important factors such as job satisfaction, commitment, organizational citizenships behaviors, withdrawal, and task performance (Colquitt, Conlon, Wesson, Porter, \& $\mathrm{Ng}, 2001)$. That being said, equity theory has several limitations that impact its applicability to compensation-related issues.

First, equity theory does not provide an easy way to make apriori predictions regarding individual levels of distributive justice. For example, internal factors such as an individual's choice of comparison standard (Goodman, 1974) or the input and outcome factors deemed to be most important to the individual (Gerhart \& Rynes, 2004) make it difficult to predict the impact of external factors on perceptions of distributive justice. Second, Adams' (1965) conceptualization of equity theory states that there are potentially negative ramifications of underpayment inequity as well as overpayment inequity. While the effects of underpayment inequity have been well documented as shown above, there is very little empirical support for overpayment inequity (Mowday, 1996). In essence, this suggests that individuals react negatively to being underpaid, but they have no problem with overpayment. Third, little is known about what type of corrective mechanisms individuals choose when they do make an attempt to correct inequity (e.g. Opsahl \& Dunnette, 1966) although these corrective actions most certainly would negatively impact the organization as a whole (e.g. Gerhart \& Rynes, 2004). In total, the literature addressing equity theory seems to suggest that individuals engage in social comparison to determine whether or not their inputs are adequately rewarded through organizational outcomes. When individuals perceive inequity, it appears that they engage in destructive behaviors such as effort withholding, undermining, and social loafing as a means of balancing the input-outcome relationship (Gerhart \& Rynes, 2004). 
Goal Setting Theory. The other dominant psychological perspective that will be discussed in this manuscript is Locke's (1968) goal setting theory. This perspective suggests that individuals that are committed to specific, measurable, achievable, realistic, time-bound, and difficult goals are more likely to exert effort and display higher levels of performance than those that make vague commitments (Locke \& Latham, 1984). Contrary to prior theories of motivation, goal setting theory suggests that compensation only motivates individuals to the extent that it influences their choice of and commitment to specific and difficult goals (Locke, 1968).

Monetary incentives are said to impact goal setting (and subsequently goal attainment and performance) in two ways: increasing the likelihood of goal acceptance and reinforcing goal commitment throughout the process (Hollenbeck \& Klein, 1987). In an update to the original theory, Locke and Latham (2002) further reinforce this notion by suggesting that practitioners design their compensation systems around individual goals in an effort to boost performance via goal acceptance and increased goal commitment. As noted by Gerhart and Rynes (2004), simply knowing that performance is based on merit is less motivational that knowing the exact level of performance at which merit bonuses are distributed. That being said, the literature addressing goal setting theory is inundated with moderating mechanisms that modify the relationship between goals, compensation, and commitment.

Over the past 45 years, numerous studies have explored the boundary conditions of goal setting theory in terms of the mechanisms through which goals, compensation, and performance interact. Of these numerous boundary conditions, goal commitment, task difficulty, feedback, and goal source are the most commonly addressed mechanisms. As first explored by Klein, Wesson, Hollenbeck, and Alge (1999), there is a multiplicative effect between goal commitment 
and goal difficulty when predicting performance. Easy goals require little effort to achieve and therefore do not motivate employees to achieve higher levels of performance. Likewise, employees are not motivated by goals to which they are not committed as this lack of commitment renders the goal inconsequential when addressing employee behavior (Gerhart \& Rynes, 2004). When combined with Locke's (1968) assertion that compensation reinforces goal commitment, these findings suggest that employees should work toward difficult goals with compensation contingent upon the attainment of those goals thus increasing goal commitment. In another vein of research, goal feedback has also been shown to moderate the relationship between goal setting and performance. Feedback can be provided by outside individuals (e.g. managers), the individual him or herself, or the task itself and it serves to keep an employee focused on the goal while also ensuring that they are making adequate progress toward the goal (Locke \& Bryan, 1969). In support of this notion, empirical evidence suggests that the existence of feedback is better than nonexistent feedback and that self-generated feedback is more motivating than external feedback (Ivancevich \& McMahon, 1982). In addition, several studies have shown that goals set by the individual him or herself are more motivating than those that are established by external sources (Wood, Atkins, \& Bright, 1999). For further support, SueChan and Ong (2002) discovered that self-set goals increased goal commitment and self-efficacy beliefs, which ultimately led to an increase in performance. In summary, compensation appears to be a useful tool for increasing commitment to difficult goals with enhanced commitment resulting from feedback and employee input in the goal setting process.

\subsubsection{Incentive Structure and Distribution}

As shown above, evidence abounds suggesting that pay-for-performance plans can provide a substantial return on investment to the organization (for meta-analytic reviews, see 
Condly, Clark, \& Stolovich, 2003; Jenkins et al., 1998; Stajkovic \& Luthans, 1997). That being said, it is important to also consider the optimal compensation structures and distribution schedules that increase the effectiveness of incentive systems. Several of the major topics addressed in this area include awarding behavior- versus results-based incentives, individual versus group rewards, merit pay versus individual incentives, and cash versus corporate sharing incentives (e.g. Gerhart \& Rynes, 2004). Each of those policy decisions have a direct impact on the utility of the incentive program, the ease with which it is administered, and employee motivation.

Outcome Measures. One of the most crucial decisions that must be made when designing a pay-for-performance plan is with regards to the use of behavioral and results-based outcome measures. Behavioral outcome measures are often based on subjective decisions made by a supervisor and are typically developed through performance management systems (e.g. Gerhart \& Milkovich, 1992). On the other hand, results-based outcome measures are directly linked to a quantitative production level. For example, a results-based outcome measure for a sales associate might correspond to a given amount of total sales for a quarter of the fiscal year. While it may seem that these measures would produce identical results, Bommer Johnson, Rich, Podsakoff, and MacKenzie (1995) discovered that the correlation between results-based and behavior-based performance measures was only .39 accounting for a total of $15 \%$ of the variance. Given this discrepancy, it is important to consider the differential effects of each option.

Many organizations prefer results-based measures of performance given that they appear to be objective (equity theory; Adams, 1965) and they draw a fairly clear link between performance and outcomes (instrumentality, Vroom, 1964). However, objective results-based measures may not always be available and they may not direct employee efforts toward the 
organization's best interests (e.g. improving output while sacrificing quality; Kerr, 1975). The primary benefit of behavior-based incentive systems is that they allow for the consideration of contextual performance in addition to task performance (e.g. Gerhart \& Milkovich, 1992). Behavior-based measures are also able to be used in any type of job, they can account for variance in performance that is out of the ratee's control, and they can focus on the process behind the results rather than simply the outcomes of the process (Gerhart \& Rynes, 2004). The primary drawbacks of behaviorally-based rating systems are the potential for inaccuracy and bias in the rating process (e.g. Heslin \& VandeWalle, 2011; Wayne, Liden, Graf, \& Ferris, 1997) and the weaker link between behavior and results (expectancy, Vroom, 1964). As shown above, there are benefit and drawbacks of choosing either results- or behavior-based incentive systems. As a result, subsequent research considered the possibility of including both results-based and behavior-based components in compensation systems. As shown by Baker and colleagues (1995) as well as Rajan and Reichelstein (2009), the combination of these two systems can result in increased expectancy, instrumentality, and employee performance. Given this evidence, it appears that situational factors, such as the availability of objective performance measures, impact the utility of both results- and behavior-based measures and performance, but the combination of the two might provide the best results.

Temporal Sequencing. The issue of merit versus individual incentives primarily addresses the timing and duration of incentive distribution. For merit incentives, participants receive a raise in base pay for achieving a desired level of long-term performance. On the other hand, individual incentives are one-time bonuses that do not impact base salary (Gerhart \& Milkovich, 1992). Merit raises are typically combined with behavior-based rating systems, which allows a manager to reward his or her subordinates for long-term performance (Gerhart \& Rynes, 
2004). This practice is beneficial to the organization in that incentive compensation can be distributed based on subjective contributions to the organization's goals and merit plans are typically more flexible in terms of cost based on the organization's ability to pay (Gerhart \& Milkovich, 1992). One of the primary flaws of merit plans is that the perceived linkages between effort, performance, and outcomes (expectancy and instrumentality; Vroom, 1964) are weaker. In addition, incentives are distributed on a check-by-check basis and do not provide the immediate psychological reinforcement to change behavior (e.g. Skinner, 1938; Pearce et al., 1985). On the other hand, individual pay plans are more directly linked to expectancy and instrumentality (Adams, 1965) and they are better for directly reinforcing positive behavior when given as a one-time lump sum. That being said, incentive plans may cause difficulties in the workplace given that they are primarily based on results-based measures (see drawbacks listed previously) and that the sole use of objective performance measures can discourage quality and contextual performance (e.g. Kerr, 1975). Another potential drawback is that performance goals for incentives may be set too low which can reduce the motivational impact of the incentive and encourage employees to reduce their performance to avoid future escalation of incentive thresholds (Whyte, 1955; Kidwell \& Bennett, 1993). In all, this evidence suggests that individual incentives are more advantageous for rewarding short-term behavior, while merit pay seems to be more advantageous for rewarding a long-term body of work. However, managers should be aware of the potential drawbacks of setting inappropriate goals and the need to show direct links between effort, performance, and rewards to reap the motivational benefits of incentive compensation.

Individual and Team Incentives. Another important factor that should be considered when designing compensation plans is the use of individual and group performance measures. 
Individual incentives are based solely on the performance of the focal individual, while group incentives are based on the output of the team as a whole (Gerhart \& Rynes, 2004). Individual incentives have long been the standard for pay-for-performance systems given that they provide a direct link between effort, performance, and rewards (expectancy and instrumentality; Vroom, 1964) that influences perceived controllability of outcomes and empowerment (Spreitzer, 1995). However, later perspectives considered the impact of group incentive plans on collective performance. Theoretical evidence would suggest that group incentives encourage members to be good citizens of the group and to develop a cohesive group that ultimately improves performance (e.g. Mullen \& Copper, 1994). However, there are also drawbacks of group incentives given the potential for social loafing (e.g. Karau \& Williams, 1993), which decreases performance via decreased investment by social loafers and groupthink (Whyte, 1989), which increases cohesion to the point the group makes illogical decisions. The logical extension of this line of research is to explore the effects of combining group and individual incentives. However, evidence from Barnes, Hollenbeck, Jundt, DeRue, and Harmon (2011) suggests that mixing individual and group incentives actually provides the individual with a social dilemma between individual and group efforts resulting in reduced overall performance. In total, these results seem to suggest that individual incentives result in more benefits and less drawbacks as compared to mixed and group incentive structures.

Forms of Incentives. The final major theme in the incentive compensation literature is the use of cash incentives as compared to corporate sharing incentives. Corporate sharing incentives include gainsharing plans, profit sharing, stock distributions, and stock options. In addition to an ownership stake via stock options, organizations can reward employees for their contributions to the organization through gainsharing (productivity of a collective unit or facility) 
or profit sharing (distributing a portion of the organization's profits to employees). The primary problem that plagues these types of programs is the distant link between employee efforts and outcomes leading to decreases in expectancy and instrumentality. Gainsharing plans are typically less susceptible to this problem given that rewards are distributed at the unit level rather than the corporate level, but nevertheless the problem still exists (Gerhart \& Rynes, 2004). On one hand, empirical evidence shows that organizations utilizing gainsharing and profit sharing plans have more productive employees, higher customer service, and higher profit margins (e.g. Banker, Lee, Potter, \& Srinivasan, 1996; Kruse, 1993). On the other hand, empirical evidence has also questioned the causality in this relationship (e.g. more profitable ventures might be more likely to use profit sharing) as well as the actual motivational benefit gained from corporate sharing incentives given the distant relationship between lower level employees and organizational outcomes (Kruse, 1993). However, evidence abounds showing the utility of corporate sharing incentives at the executive level (e.g. Gomez-Mejia \& Wiseman, 1997; Nyberg, Fulmer, Gerhart, \& Carpenter, 2010) including several meta-analyses (e.g. van Essen, Heugens, Otten, \& van Oosterhout, 2012; Tosi, Werner, Katz, \& Gomez-Mejia, 2000). This total body of evidence seems to suggest that corporate sharing incentives are highly motivating at the executive level, but their utility is questionable at lower levels of the organization.

Incentive Intensity. To summarize the above, we know that compensation can be used to motivate employees and that its motivational value is dependent upon design factors of the compensation system. However, one crucial question still remains: How much incentive compensation do we need to provide in order to reap these benefits? To address this issue, scholars have studied the effect of incentive intensity, the overall size of the incentive as compared to base pay (Garbers \& Konradt, 2014), on employee motivation. To date, most of the 
research addressing this construct is contained within the teams literature (e.g. Messersmith, Guthrie, Ji, \& Lee, 2011; Zenger \& Marshall, 2000). As with most other topics in the compensation literature, scholars began by addressing the impact of excessive intensity as compared to insufficient intensity.

Insufficient incentive intensity occurs when the amount of the incentive or the proportion of the incentive amount as compared to total compensation is not sufficient for adequately motivating the recipient. On the other hand, excessive intensity occurs when individual incentives are powerful enough to reduce cooperation and create intense competition between employees that may run counter to the best interests of the organization (e.g. Pfeffer \& Langton, 1993). Research addressing these issues has generally found that strong incentive intensity can substantially boost performance (e.g. Jenkins et al., 1998; Zenger \& Marshall, 2000). However, larger differences in compensation for individuals in similar jobs can also reduce collaboration and impact risk-taking as success or failure could result in a substantial decrease in total compensation (Pfeffer \& Langton, 1993). Given these benefits and drawbacks, it is widely considered that high intensity incentive programs are "high-risk, high-return" strategies (Gerhart, Trevor, \& Graham, 1996). Supporting this statement, Milgrom and Roberts (1992) suggested that the optimal incentive intensity is dependent upon incremental profit that can be obtained from increased effort, the ability to precisely measure the activities that contribute to profitability, risk tolerance, and employee responses to incentives. Under these conditions, strong incentives should increase employee effort (and subsequently performance) while controlling the level of risk assumed by the organization. 


\section{CHAPTER 3: HYPOTHESIS DEVELOPMENT}

\subsection{Thin Slices of Behavior in Employment Interviews}

As noted earlier, both researchers and practitioners agree that applicants and interviewers often meet informally before engaging in a formal interview (e.g. Ulrich \& Trumbo, 1965; Barrick et al., 2010). The first such meeting often occurs at a career fair or professional meeting in which the interviewer engages with potential applicants for short periods of time (typically 3-5 minutes). Career fairs are events designed for exchanging information in which multiple companies gather in a common location and interact with job seekers (Cable \& Yu, 2006). These interactions give the recruiter time to review the potential applicant's resume while both parties can also engage in rapport building and exchange information in a low commitment environment. Organizations often choose to utilize career fairs as a means of advertising job openings, performing pre-interview screening, and increasing the richness of information that is presented to applicants as compared to more static channels such as websites and online job boards (Cable \& Yu, 2006). In the context of thin slicing, interactions at a career fair would typically be termed as a thin slice of behavior given that interactions typically last less than 5 minutes (Ambady \& Rosenthal, 1992).

In addition, another thin slice of behavior is observed by the interviewer at the time of the actual interview. Interviewers and applicants often exchange in brief rapport-building sessions to familiarize themselves with one another and decrease tension before beginning the interview (Chapman \& Zweig, 2005). Information exchanged during this initial rapport-building session tends to gravitate toward personal interests, hobbies, and other light conversational topics that are not closely related to the job opening or the organization (Chapman \& Zweig, 2005). Data 
collected from a pilot study utilizing mock interviews suggests that this period typically lasts for approximately 90 to 120 seconds. This brief exchange is often followed by a broad interview question asking the applicant to describe why he or she wishes to join the organization (e.g. Conway \& Peneno, 1999). This broad question serves as an additional measure to make the applicant feel comfortable, while also gathering task-relevant information regarding the applicant's motivations, qualifications, and preparation for the interview (Conway \& Peneno, 1999). Additional pilot data assessing the duration of this interaction suggests that this exchange typically lasts between 110 and 150 seconds. In total, this initial interaction to begin the interview should reach a duration of between 200 and 290 seconds, suggesting that it would be classified as a thin slice of behavior (Ambady \& Rosenthal, 1992).

In essence, the above information suggests that interviewers are often exposed to two separate thin slices of behavior before engaging in the formal employment interview. One of the major shortcomings of the existing thin slicing literature is the relatively nonexistent literature addressing the ability of individuals to store and recall information gained from observing thin slices of behavior. For example, many studies have shown the predictive validity of thin slices when perceptions of the focal actor are measured immediately after exposure to thin slices of behavior (e.g. Ames et al., 2010; Carney et al., 2007; etc.). In addition, other research has compared the validity of thin slices with thicker slices for predicting outcomes showing support for the thin slicing construct (e.g. Ambady \& Rosenthal, 1993; Borkenau et al., 2004). However, researchers have not fully explored the validity of thin slices for predicting decision outcomes that are temporally separated from the observation of behavior. In a selection context, this is applicable in that impressions formed during career fairs are temporally disjointed from the preinterview rapport building and structured interview. 
The existing literature addressing thin slices of behavior suggests that observers rapidly gain information within the first few moments of an interaction (Ambady \& Rosenthal, 1992). In addition, existing evidence suggests that this information is collected via automatic processing with time being a crucial factor when making the shift from intuitive to analytic processing (e.g. Ambady, 2010; Vallacher \& Wegner, 1987). These features of the thin slicing construct suggest a need to address the storage and recall of information gained during thin slice observations. Given that this topic has yet to be directly explored, existing theory can help to inform inquiry on this topic. Fuzzy trace theory (Brainerd \& Reyna, 1990) addresses the storage and recall of information gathered when observing thin slices of behavior when addressing gist extraction, the fuzzy processing preference, and reconstruction of short-term memory. Gist extraction suggests that individuals automatically distill precise information down to its basic meaning to reduce cognitive load (Brainerd \& Reyna, 1990). This would suggest that individuals subconsciously simplify information gained during thin slices during both career fairs and pre-interview rapport building so that it can be efficiently deposited in long-term storage for use in future processing. In addition, the fuzzy processing preference suggests that individuals prefer to process information at the least precise level that he or she deems to be acceptable (Brainerd \& Reyna, 1990). This should aid in the storage and future recall of information gained from thin slices given that individuals are able to develop a more holistic representation of the interaction. In sum, the literature addressing thin slices of behavior and the extensions provided by fuzzy trace theory suggest that initial impressions will ultimately impact subsequent impression.

\section{H1: Initial impressions of employability formed at the career fair will positively impact} second impressions formed at the beginning of the interview. 
In line with the above hypothesis, it seems likely that the second impressions formed at the beginning of the interview will positively impact both interview scores and final impressions of employability. As noted earlier, the second impression formed during the first few minutes of the interview is primarily comprised of two components: a general rapport-building session that is not task-related and a broad initial interview question that is highly task-related. The general rapport-building information should positively relate to information collected during the initial interaction as both participants reintroduce themselves and exchange basic information. As noted in fuzzy trace theory, cognition falls on a continuum between verbatim (exact details) to gist processing (basic, bottom line meanings) with individuals holding a natural inclination toward processing at the gist end of the spectrum (Brainerd \& Reyna, 1988). In addition, fuzzy trace theorists also suggest that task-relevant information is processed closer to the verbatim end of the continuum than non-task relevant information (see Figure 3 for a pictorial representation). This notion is also supported by the dual processing perspective in that the addition of task-related information should further activate System 2 processing, which would allow the individual to be more efficient when gathering information. This should allow the interviewer to collect new information that was not presented or collected in the initial interaction.

To complement the fuzzy trace perspective, the literature addressing thin slices of behavior also supports the notion that information collected in the first few minutes of the interview encounter should predict both interview scores and final impressions of employability (e.g. Ambady \& Rosenthal, 1992). Numerous studies have shown that impressions formed within the first several minutes of an interaction predict final decision outcomes in settings such as supervisor performance ratings (Blanck et al., 1986), salesperson effectiveness (Ambady et al., 2006), and negotiation decisions (Curhan \& Pentland, 2007). In addition, several studies have 
shown that initial impressions strongly predict impressions developed during thicker slices of exposure (e.g. Ambady \& Rosenthal, 1993; Borkenau et al., 2004; Curhan \& Pentland, 2007). All of this information suggests that interviewers collect a significant amount of information that informs both their interview scores and final hiring decisions within the first several minutes of the interview interaction.

H2: Second impressions of employability formed at the beginning of the interview will positively impact the applicant's structured interview score.

H3: Second impressions of employability formed at the beginning of the interview will positively predict final impressions of employability.

H4: Second impressions of employability formed at the beginning of the interview will mediate the relationship between initial impressions formed at the career fair and final impressions of employability.

When considering the impact of interview scores on final ratings of employability, there is very little disagreement in the existing literature that the two constructs are strongly and positively correlated (e.g. Huffctutt \& Culbertson, 2010; Lievens et al., 2005). A vast literature addresses the link between interview scores and hiring outcomes with consistent and strong findings that interview scores predict employment offers (e.g. Arvey \& Campion, 1982; Barrick et al., 2010). In addition, many studies employing unstructured interviews often use interview outcomes as a proxy for decision outcomes given that the outcome is almost entirely subjective with little consistency between interviewers (e.g. Hunter \& Hunter, 1984; Huffcutt \& Arthur, 1984). However, the rigidity of structured interviews can create separation between interview scores and perceptions of employability as structured interviews increase consistency between 
interviewers, but may not capture the interviewer's total perceptions of employability (e.g.

Macan, 2009). As a result, it seems reasonable to expect that there will be a strong significant relationship between interview scores and final ratings of employability.

H5: Structured interview scores will positively predict final impressions of employability.

H6: Structured interview scores will mediate the positive relationship between second impressions formed at the beginning of the interview and final impressions of employability.

As a caveat to the above statements, work in other areas suggests that interview scores may share a substantive amount of predictive variance with initial impressions formed during the first few minutes of the interview interaction. Scholars exploring the thin slicing construct have routinely found that increasing the length of interactions beyond the bounds of thin slicing does not typically produce large amounts of new information (e.g. Ambady \& Rosenthal, 1993; Borkenau et al., 2004; Curhan \& Pentland, 2007; Murphy, 2005). This is very important in an employment interview context in that recruiters may gather a substantial portion of the information that they use to make final employment decisions within the first several minutes of interacting with a job candidate. This possibility is further supported by the dual processing literature, which suggests that System 1 and System 2 processing can work together to process large quantities of information and make sequential decisions quickly (e.g. St. B. T. Evans, 2010; Thompson, 2009).

These components are combined in the fuzzy trace literature to suggest that individuals can efficiently process task-related information to make quick and accurate decisions (e.g. Brainerd \& Reyna, 1988; Reyna et al., 1990). Additionally, work addressing the fuzzy 
processing preference suggests that individuals prefer to store and process information at the least precise levels deemed necessary by the decision maker (Reyna et al., 1988). This helps to explain the findings of researchers addressing the anchoring heuristic (e.g. Tversky \&

Kahneman, 1974) by suggesting that individuals make judgments about others based on initial impressions and that those early impressions are very difficult to modify (e.g. Butler, 1986; Cervone \& Peake, 1986). In total, this evidence suggests that individuals develop strong initial impressions that ultimately account for a large portion of the variance in final employability ratings. In addition, evidence also suggests that increasing interaction durations to the lengths of a traditional employment interview (30 minutes to 1 hour) makes less of an impact on final ratings of employability as compared to initial impressions formed during the second impression at the beginning of the interview (e.g. Ambady \& Rosenthal, 1993; Curhan \& Pentland, 2007).

H7: The direct effect of second impressions of employability formed at the beginning of the interview on final ratings of employability will be significantly stronger than the direct effect of structured interview scores on final ratings of employability.

\subsection{The Effect of Confidence on Decision Stability}

Decision confidence is one of the factors that is hypothesized to modify the relationship between initial impressions and subsequent impressions. First, this assertion is supported by work addressing self-efficacy, which suggests that individuals that are confident in their abilities are more likely to achieve higher levels of performance (e.g. Judge \& Bono, 2001; Stajkovic \& Luthans, 1998). In the context of this manuscript, self-efficacy should increase both confidence in decisions and performance as interviewers higher in self-efficacy would benefit from both perceived and actual increases in decision performance. 
Second, other research also suggests that confidence could also reinforce initial decision outcomes through mechanisms such as cognitive anchoring and confirmation biases. A vast literature has addressed the interplay between confidence and decision stability over time with recurring themes including systematic overconfidence across all individuals (e.g. Lichtenstein, Fischhoff, \& Phillips, 1982; Yates, 1990) and that confidence levels remain relatively stable over time even after the introduction of new information (e.g. Jonsson \& Allwood, 2003). This suggests that overconfidence could encourage individuals to retain their initial impressions even after the introduction of new information. In addition, other factors such as cognitive anchoring and confirmation biases also suggest that confidence in an initial decision can ultimately impact future decisions.

Cognitive anchoring occurs when individuals rely on initial information and then adjust their perceptions as interpersonal interactions progress (Tversky \& Kahneman, 1974). This ultimately results in final decisions that are moderately biased in the direction of the initial anchor (Bazerman, 1990). In addition, increased confidence in initial anchors (i.e. judgments based on initial interactions) further strengthens the relationship between initial decision outcomes and final decision (e.g. Schmitt, 1976; Simmons \& Nelson, 2006). Additional support is provided by the literature addressing confirmatory biases, which suggest that individuals are biased toward recalling information that supports their initial assessment of another individual (Murphy, Gannett, Herr, \& Chen, 1986). Research in this area suggests that confirmatory biases are strengthened as confidence increases because confidence in initial decisions encourages individuals to make consistent decisions over time (e.g. Tsai, Klayman, \& Hastie, 2008). All of this evidence suggests that confidence in a given decision should strengthen the relationship between concurrent decision outcomes and subsequent decision outcomes. 
H8a: Confidence in employability ratings after developing an initial impression formed at the career fair will moderate the relationship between initial impressions formed at the career fair and second impressions formed at the beginning of the interview, such that the relationship between initial impressions formed at the career fair and second impressions formed at the beginning of the interview will be stronger as confidence in initial impressions formed at the career fair increases.

H8b: Confidence in second impressions formed at the beginning of the interview will moderate the relationship between second impressions formed at the beginning of the interview and structured interview scores, such that the relationship between second impressions formed at the beginning of the interview and structured interview scores is stronger as confidence in second impressions formed at the beginning of the interview increases.

H8c: Confidence in second impressions formed at the beginning of the interview will moderate the relationship between second impressions formed at the beginning of the interview and final impressions of employability, such that the relationship between second impressions formed at the beginning of the interview and final impressions is stronger as confidence in second impressions formed at the beginning of the interview increases.

\subsection{The Impact of Incentive Compensation}

As noted extensively in the literature addressing employee compensation (e.g. Jenkins et al., 1998; Stajkovic \& Luthans, 1997), incentive compensation can be used to motivate employees which can ultimately result in increased employee performance and a return on 
investment for the organization. In addition, the literature addressing executive compensation has firmly established that incentive compensation can be used to modify executive decision making processes in order to align the interests of the individual with the interests of the organization (e.g. Gomez-Mejia \& Wiseman, 1997; Martin et al., 2013; Pepper \& Gore, in press). However, the current literature addressing the effect of incentive compensation on non-executive decision makers is much less developed. These individuals make daily decisions that impact organizational performance, competitive position, and human capital (Becker \& Gerhart, 1996; Floyd \& Wooldridge, 1997). For this reason, it is important to consider the impact of incentive compensation on the decision processes of non-executive decision makers.

When considering the motivational influence of compensation, goal setting theory is a useful framework for exploring the increased attention and focused effort that results from incentive compensation. These mechanisms increase performance in that incentive compensation increases goal acceptance and commitment through increased attention and effort, which ultimately aids in meeting the goal (Klein et al., 1999). In this way, realistic, achievable, and specific goals that are aligned with the organization's best interests and reinforced with incentive compensation can be a powerful motivational tool. In a recruiting context, aligning goals and compensation with recruiter effectiveness metrics such as new hire quality, retention, offeracceptance ratio, or supervisor performance evaluations should increase the recruiter's attention and commitment toward finding highly qualified applicants and accurate assessment.

In the context of non-executive decision makers, compensation can also increase the efficiency and effectiveness of decision making by increasing cognitive load. Cognitive load theory suggests that learning is impacted by the amount of mental load and mental effort that is present during processing (Sweller, 1988). In most instances, past research has focused on 
situations in which participants are bombarded with erroneous information about a non-focal task that impacts their ability to absorb information about the focal task itself (e.g. Fiske \& Taylor, 1984). This creates a condition of cognitive overload, which is detrimental to learning and information retention (e.g. O’Reilly, 1980). On the other hand, Paas, Renkl, and Sweller (2004) also suggest that individuals that are not fully invested in learning or collecting information can experience cognitive underload where the participant is not reaching his or her full potential in terms of cognitive processing. Both of these conditions can lead to ineffective decision making as the decision maker is failing to retain useful information in both cases. In most cases, cognitive overload occurs when individuals are distracted from completing the focal task when they experience cognitive demands emanating from other sources (Sweller, 1988). Cognitive underload is likely to occur in situations where the focal actor is not adequately motivated to collect and store information for future processing (Paas et al., 2004). As noted earlier, compensation can be used to increase attention and effort toward the pursuit of specific and achievable goals. This should discourage cognitive underload while also encouraging decision makers to collect and store useful information that can be applied in further cognitive processing.

Based on the above information, it seems reasonable to predict that the effects of fuzzy trace theory will be magnified via incentive compensation and focus attention toward important goals. As a result of increased attention and cognitive load, decision makers should be able to extract additional gist information that can easily be moved to long-term storage. In addition, participants should also be more prepared for reconstructive retrieval of verbatim traces given that they would have more stored memory points to use when "working backward." This should be especially important during exposure to thin slices of behavior as the brevity of the interaction necessitates optimum cognitive performance in order to extract accurate and useful information 
from the encounter. In short, incentive compensation should strengthen the relationship between initial impressions and subsequent impressions as a result of increased goal commitment, cognitive load, and gist information extraction.

H9a: Recruiter incentive compensation will moderate the relationship between initial impressions formed at the career fair and second impressions formed at the beginning of the interview such that the relationship between initial impressions formed at the career fair and second impressions formed at the beginning of the interview will be stronger under a pay-for-performance condition than a fixed compensation condition.

H9b: Incentive compensation will moderate the relationship between second impressions formed at the beginning of the interview and structured interview scores such that the relationship between second impressions formed at the beginning of the interview and structured interview scores will be stronger under a pay-for-performance condition than a fixed compensation condition.

H9c: Recruiter incentive compensation will moderate the relationship between second impressions formed at the beginning of the interview and final impressions of employability such that the relationship between second impressions formed at the beginning of the interview and final impressions of employability will be stronger under a pay-for-performance condition than a fixed compensation condition.

Insert Figure 4 About Here 
Insert Figure 5 About Here

- - - - - - - - - - - 


\section{CHAPTER 4: METHOD}

\subsection{PARTICIPANTS}

Data were collected in conjunction with a comprehensive interview simulation conducted at a large mid-Atlantic university in the United States. The applicants received course credit for participating in this study. In addition, they were also entered into a drawing for an incentive in exchange for their voluntary permission to use their survey responses for the purposes of this manuscript. Each applicant received training prior to the experiment to familiarize him or herself with the STAR (Situation or Task, Action, and Result) interview strategy, in addition to coaching regarding proper résumé, cover letter, and interview preparation. Given that these participants were sophomores and juniors that were searching for internships, they represent members of the population that this study attempts to address. The interviewers were trained graduate students with training equivalent to that of the average graduate of a master's degree program in human resource management. In addition, each interviewer participated in a pilot experiment in which they interviewed 7 to 9 applicants and they will had at least 3 months of work experience in the human resource management field. In total, 229 applicants and 28 interviewers participated in this experiment. Given the nature of this study, applicants were screened for prior relationships with the interviewer to prevent the misinterpretation of initial impressions in these situations. However, no such prior relationships existed between interviewers and applicants.

\subsection{EXPERIMENTAL DESIGN}

To address external validity, the hiring procedures for this experiment were developed in collaboration with a global Fortune 50 corporation with the goal of selecting new graduates for a leadership development program. After receiving a job description and the pre-experiment 
training as described above, interviewers and applicants met for the first time at a simulated career fair where interviewers established rapport with the applicants and discussed the logistics of the process. Applicants were first given information from the experimental administrator describing the job itself and the basics of the hiring process for the experiment. They were then given several minutes with their interviewer to speak informally and to schedule an interview. Upon the conclusion of this meeting, both parties completed a pre-interview survey that contained several personality and individual difference scales that were used for the purposes of this study as well as to generate a developmental report for each participant that were distributed after the completion of this experiment. Interviewers also completed an assessment for each applicant to assess the perceived employability of each applicant as well as the interviewer's confidence in that assessment immediately after the simulated career fair.

Approximately two weeks after completing the steps described above, participants engaged in thirty minute structured employment interviews. Interviewers were given several minutes to build rapport with the applicant before the structured interview began. After this brief rapport-building session and a broad initial interview question, interviewers again completed a brief scale to assess the perceived employability of each applicant as well as their confidence in that assessment. The structured interview questions that followed resulted in a behavioral interview score for each applicant that was based on the content and quality of their answers.

Upon completion of this process, the interviewers completed the employability and confidence scales for a final time to give their final recommendation to the "company." Although data collection for this study ended at that point, the experiment continued through several rounds of decision making and ultimately concluded with the "hiring" of the top ten percent of all applicants that participated in the simulation. This was done to increase the realism of the 
experiment by closely emulating the entire hiring process of a Fortune 50 corporation and inviting those that were "hired" to visit with human resource personnel for the sponsoring organization. In all, this design should provide external validity given that the experiment was strictly based on the hiring processes of a large global organization while using members of the population to which this study might generalize. In addition, the lab-based nature of the study allowed for experimental control with regards to temporal sequencing of data collection and limitation of outside interaction, increasing the internal validity of inferences drawn from the results.

\subsection{COMPENSATION MANIPULATION}

As noted in the hypothesis development section, one of the goals of this manuscript is to assess the impact of incentive compensation on decision making among non-executive employees. For this reason, interviewers were exposed to an incentive compensation manipulation. Each recruiter received fixed base pay at a rate of ten dollars per hour. They were then randomly assigned to one of two incentive compensation conditions. In the first condition (Region A), all participants received a twenty five dollar gift card for their participation in the study regardless of their performance. This does not constitute incentive compensation as there is no variable component to the compensation structure (Gerhart \& Rynes, 2004). In the second condition (Region B), recruiters had the opportunity to earn a one hundred dollar gift card if they were selected as one of the top recruiters in their region. Incentive compensation was distributed based on professionalism, work quality as assessed by the administrator of the experiment, and applicant reactions. The one hundred dollar incentive amount was chosen based on the incentive intensity literature (e.g. Garbers \& Konradt, 2014; Zenger \& Marshall, 2000). This literature suggests that incentives can be detrimental when they are either insufficient to change behavior 
or when an incentive amount is so high that it encourages excessive risk-taking or collaboration (e.g. Pfeffer \& Langton, 1993). For these reasons, the incentive amount increased base pay by 35 to 40 percent. While this might be a larger percentage than would commonly be encountered for actual interviewers, it was necessary in this experiment as a function of the brevity (and therefore lower total compensation) of this experiment. This should produce enough intensity to encourage interviewers to perform to the best of their abilities without engaging in excessive risk-taking or sabotage of other employees.

\subsection{MEASURES}

\subsubsection{Perceptions of Employability}

Perceptions of employability were measured using a 5-item, 7-point Likert scale developed by Krefting \& Brief (1976) and adapted by Herold (1995). This measure was designed to assess perceptions of the applicant's ability to do the job, potential for quality and quantity output, ability to get along with others, and potential for promotion. Past research has shown that this scale provides a stable employability factor with adequate consistency ( $\alpha=.87$; Herold, 1995). Internal consistency statistics from this experiment provided slightly higher levels of consistency after the career fair $(\alpha=.93)$, the second impression $(\alpha=.96)$, and final impressions $(\alpha=.95)$ of employability as compared to prior studies.

\subsubsection{Rating Confidence}

Following past research (e.g. Garb, 1989), rating confidence was assessed on a 5-point Likert scale anchored by "Not Confident" and "Highly Confident." Interviewers completed this confidence item in response to each item on the employability scale. This resulted in a 5-item confidence scale that assessed total confidence in employability assessments. Confidence 
measures collected after the career fair $(\alpha=.93)$ and second impressions $(\alpha=.92)$ showed very high levels of internal consistency.

\subsubsection{Structured Interview Scores}

Structured interview scores resulted from the structured interview based around the practices of a Fortune 50 organization as well as Huffcutt and Arthur's (1994) interview structure typology. The interview consisted of 8 questions that utilized both behavioral and situational structure as suggested in the existing literature (e.g. Huffcutt et al., 2001). The interview questions are included on the structured interview form in Appendix A and include questions such as "Give me an example of a good decision you have made recently. What alternatives did you consider and why did you choose one alternative over the others?" In terms of the amount of structure for the interview, the design for this study fell into Structure III of Huffcutt and Arthur's (1994) nomenclature displayed in Figure 2. Interviewers were required to ask every question, but they had the opportunity to probe for additional information if they chose to do so. In addition, interviewers had a free response area to take notes on the interview form and they scored each answer on a 4-point Likert-type behaviorally anchored rating scale to improve the validity and reliability of interview scoring (e.g. Jacobs et al., 1980; Kingstrom \& Bass, 1981). The eight questions used on the structured interview form showed adequate internal consistency $(\alpha=.83)$.

\subsection{CONTROL VARIABLES}

\subsubsection{Gender Congruence}

Gender congruence was included as a control variable to address gender biases that are inherent in employee selection (e.g. Graves \& Powell, 1995). Data were collected from both the 
interviewer and the applicant with respondents selecting " 0 " for female and " 1 " for male. The data were then recoded into one dichotomous dummy code that were coded " 0 " for gender incongruence and "1" for gender congruence.

\subsubsection{Race Congruence}

In addition to gender bias, a control for the "like me" bias via race was also included in this study (e.g. Garcia et al., 2008; Huffcutt \& Roth, 1998). This study utilized the classifications employed by the United States Census Bureau. These classifications include: "American Indian or Alaska Native," “Asian," Black or African American," "Hispanic or Latino," "Native Hawaiian or Other Pacific Islander," "White," and "Other." After collecting this information from both interviewers and applicants, the results were dummy coded "0" for race incongruence and "1" for race congruence.

\subsubsection{Applicant Objective Qualifications}

Another factor that could influence the relationship between initial impressions and subsequent impressions is the applicant's objective qualifications. Applicant factors such as GPA and work experience could substantively impact decision making in that the interviewer might look favorably upon applicants with high GPAs and extensive work experience causing their ratings of employability to be more stable over time. For this reason, work experience was measured on a five-point Likert scale with markers of " 0 years of experience," "less than 1 year of experience," "1-2 years of experience," "2-3 years of experience," and "more than 3 years of experience." GPA was also measured on a five-point Likert scale with markers of " $<2.0$," "2.01 to 2.5, ," $2.51-3.0$, , "3.01-3.50," and "3.51 to 4.0." 


\subsection{ANALYTIC STRATEGY}

Given the nature of the model specified in this manuscript, there are several important considerations that were acknowledged when developing an analytical strategy. First, the data in this experiment are nested given that each interviewer conducted multiple interviews. Second, this design involved additional nesting via repeated measures in that both employability and rating confidence were measured at multiple points in time. Third, the chain of moderated and mediated relationships specified in the model encouraged the use of full information estimation techniques that allow for the simultaneous estimation of parameters and assessment of overall model performance. These considerations informed the decisions made when developing the analytical strategy below.

Given these considerations, multilevel structural equation modeling (ML-SEM) utilizing the Mplus platform (Muthén \& Muthén, 2012) version 7.2 was identified as the most appropriate tool for testing the proposed model. This analytical tool utilizes maximum likelihood estimation while also allowing for separate partitioning of within-level (variance that is unaffected by interviewer) and between-level (variance among applicants that is due to interviewer differences) variance by creating separate variance-covariance matrices for each level of analysis. This avoids the commission of atomistic and ecological fallacies when analyzing the data and interpreting the findings. In addition, Mplus utilizes a Huber-White sandwich estimator (Huber, 1967; White, 1980) to create cluster robust standard errors that are not skewed by the multilevel nature of the data. This allows for accurate significance testing when addressing both within-level and acrosslevel phenomena. 
A single indicator model that accounts for random measurement error was used given the complexity of the proposed model. This complexity is a result of the utilization of multilevel data with multiple latent interactions and multiple stages of mediation. Following the guidance of Kenny (1979) as outlined by Williams and Hazer (1986), factor loadings from indicator to construct were fixed to the square root of the alpha reliability statistic with respective error terms fixed to one minus the alpha reliability statistic multiplied by the variance. As shown by Netemeyer, Johnston, and Burton (1990), this design produces parameter estimates and significance testing similar to that of a latent variable model utilizing multiple indicators that is far superior to a single indicator model that does not incorporate random measurement error. Due to the complexity of the model, the hypotheses were tested using several different analyses to avoid empirical underidentification and power-related issues. The first model addressed hypotheses one through six to assess the general stability of impressions over time. The second model tested hypothesis seven utilizing a Satorra-Bentler scaled chi square difference test (Satorra, 2000) to assess the relative importance of second impressions and interview scores when predicting final impressions of employability. The third and fourth models address the moderating effects of decision confidence and incentive compensation respectively.

Several fit statistics were used to judge the fit of a specified model to the data. First, a chi square test was performed to assess absolute fit. This statistic is reported in nearly all SEM studies and does not consider the improvement of the specified model as compared to the null model which contains no structural paths. However, the results of the chi square test should be interpreted cautiously as it is very sensitive to sample size (more observations increases the likelihood of a significant chi square), number of paths estimated, covariance magnitudes, and deviations from normality. Second, I examined the comparative fit index (CFI), which is an 
incremental fit index that assesses the improvement of the specified model as compared to an absolute null model specifying no relationships among the observed variables. As guided by $\mathrm{Hu}$ and Bentler (1999), a CFI value of .95 or greater indicates good fit for the specified model. Third, the root mean square error of approximation (RMSEA) was examined. The RMSEA is an absolute fit index that is adjusted for parsimony and should not exceed .06 to indicate good fit (Hu \& Bentler, 1999). Fourth, the standardized root mean residual (SRMR) was examined. The SRMR is an absolute fit index that compares the residuals in the implied covariance matrix with the residuals in the observed covariance matrix. As specified by Hu \& Bentler (1999), SRMR values should not exceed .08 to indicate good fit. It is important to note that several of these fit statistics are negatively impacted by factors such as sample size, model complexity, and covariance sizes. For this reason, it is useful to employ a battery of fit statistics rather than relying on a single fit statistic.

Another important consideration is the testing of hypothesis 7, which suggests that the effect of second impressions formed at the beginning of the interview will be significantly stronger than the structured interview score when predicting final ratings of employability. This hypothesis was tested by placing equality constraints on the paths between both second impression and interview scores and final ratings of employability. A nested model comparison was then conducted via a Satorra Bentler scaled chi square difference test (Satorra, 2000) to determine the statistical significance of including the equality constraint. A significant test of the equality constraint would indicate that the two focal relationships significantly differ in magnitude with the relationship.

While the above model allowed for testing of the first eight hypotheses, hypotheses $9 \mathrm{a}$, $9 \mathrm{~b}$, and $9 \mathrm{c}$ required testing at the interviewer level of analysis. However, the fact that the 
compensation variable is dichotomous (incentive compensation vs. fixed compensation) allowed for testing at the interviewer level without including a new variable at the interviewer level of analysis. To accomplish this task, a multiple groups analysis was conducted assessing interviewers in the incentive compensation condition and those that received fixed compensation. This analysis was conducted by freely estimating the paths of interest in one model and constraining the paths of interest to be equal in the other (paths of interest are specified in hypotheses 9a, 9b, and 9c). A significant Satorra Bentler scaled chi square difference test (Satorra, 2000) would indicate that constraining the paths to be equal is too costly in terms of fit. A non-significant chi square difference test would suggest that we should accept the more parsimonious model that constrains the paths of interest. It was expected that the incentive compensation model will produce significantly stronger relationships between employability ratings than the fixed compensation model. In the event that this omnibus test is significant, a multiple groups analysis would be conducted individually for each relationship specified in hypotheses 9a, 9b, and 9c. This allowed for closer examination as to which individual relationships are moderated by incentive structure. In the event of significant results for hypotheses 9a, 9b, and 9c, results obtained for the other eight hypotheses would be reinterpreted given the use of a combined sample (including both incentive and fixed compensation conditions) for testing.

When testing the above model, it was important to consider control variables that could impact the proposed relationships. First, it was important to consider that within-group and between group variance were separately estimated when employing the ML-SEM estimation routine. The Huber-White sandwich estimator helped control for interviewer effects such as leniency and strictness biases, which lessened the need for interviewer-level control variables. 
Second, it was important to consider objective applicant qualifications that could substantively impact interviewer decision making. For this reason, applicant GPA and work experience were included as control variables in the model. Third, it was important to consider the "like me" bias that often occurs in employee selection (e.g. Garcia et al., 2008). For this reason, both gender and race congruence were included as control variables in the final model. These control variables should account for the effect of interviewer biases and objective applicant qualifications on employment decisions. 


\section{CHAPTER 5: RESULTS}

\subsection{DESCRIPTIVE STATISTICS AND CORRELATIONS}

Means, standard deviations, and correlations for study variables are included in Table 4 below. Of note, the average GPA among applicants was between 2.5 and 3.5 with an average of 1-2 years of work experience. Among the variables of interest, we see relatively high correlations between employability ratings and interview score across time indicating some level of temporal stability. In addition, we also see an increase in standard deviation among employability scores across time suggesting that recruiters provide more differentiation among applicants as time progresses. All significant correlations were in the expected direction, with most hypothesized relationships showing significant correlations. Of note, the relationship between initial impressions of employability and second impressions of employability appears to be weaker $(r$ $=.227$ ) than the other correlations between employability ratings (second impressions-interview score $r=.461$; interview score-final impressions $r=.666$; second impressions-final impressions $r=.713)$.

Insert Table 4 About Here

\subsection{BASELINE MULTILEVEL STRUCTURAL EQUATION MODEL}

As discussed in Chapter 4, the first analysis was a baseline ML-SEM to test the first six hypotheses. These hypotheses address the stability of employability ratings over time including initial impressions of employability, second impressions of employability, interview scores, and 
final impressions of employability. Results of this analysis are reported in Table 5. Overall, the integrity of the baseline model was supported by the overall fit statistics. The overall model displayed a relatively low chi square value $(\chi 2=14.625 ; d f=7 ; p<.05)$ despite being statistically significant. In addition, the RMSEA just slightly exceeded the .06 threshold suggested by $\mathrm{Hu}$ and Bentler (1999) at .069 . However, the RMSEA being extremely close to Hu and Bentler's (1999) coupled with the repeated discussion of the inadequacy of precise cutoff values (e.g. Cohen, 1994; Marsh, Hau, \& Wen, 2004) suggests that we should explore other fit indices to further describe the overall fit of the model. The SRMR for this model was .049, which is below the $\mathrm{Hu}$ and Bentler (1999) cutoff of .08 and indicates good fit. In addition, the CFI for the model was .976, which exceeds Hu and Bentler's cutoff of .95 indicating good fit. In total, this evidence suggests that there is adequate fit for the overall model structure.

Insert Table 5 About Here

Hypothesis 1 suggested that impressions formed at the career fair would positively impact second impressions of employability formed at the beginning of the interview. As shown in Table 5, this hypothesis was not supported as evidenced by the non-significant parameter estimate $(\beta=-.010$, n.s. $)$ and lack of explained variance $\left(R^{2}=.000\right)$. This suggests that impressions formed during the career fair do not impact subsequent impressions developed at the beginning of the interview. Hypothesis 2 suggested that second impressions formed at the beginning of the interview would positively impact the applicant's structured interview score. This hypothesis was supported by a significant parameter estimate $(\beta=.589 ; p>.001)$ with 
second impressions explaining $34.7 \%$ of the variance in interview scores. The third hypothesis proposed that second impressions formed at the beginning of the interview would positively predict final impressions of employability. This hypothesis was also supported by a significant parameter estimate $(\beta=.346 ; p>.001)$ with second impressions of employability explaining $12 \%$ of the variance in final impressions of employability. The fourth hypothesis proposed that second impressions formed at the beginning of the interview would mediate the relationship between initial impressions formed at the career fair and final impressions of employability. This hypothesis was tested using the Monte Carlo method for assessing mediation (MCMAM; MacKinnon, Lockwood, \& Williams, 2004). Results of the bias corrected boostrapped estimate of the indirect effect failed to support this hypothesis ( $\mathrm{p}=-.0081 ;$ n.s.; $95 \%$ CI [-.205, .189]). All of this evidence suggests that initial impressions formed during the career fair do not impact further impressions of employability, but second impressions formed at the beginning of the interview make a strong impact on interview scores and final impressions of employability.

The fifth and sixth hypotheses address the interview score's impact on final impressions of employability. Hypothesis 5 asserted that the structured interview score would positively predict final impressions of employability. This relationship was supported by a significant parameter estimate $(\beta=.674 ; p>.001)$ with interview scores explaining $45.4 \%$ of the variance in final impressions of employability. The sixth hypothesis suggests that structured interview scores will mediate the relationship between second impression formed at the beginning of the interview and final impressions of employability. This hypothesis was tested using MCMAM (MacKinnon et al., 2004) which provided support using a bias corrected bootstrapped estimate of the indirect effect $(\mathrm{p}=.519 ; p>.05 ; 95 \%$ CI $[.348, .690])$. 


\subsection{STRENGTH OF SECOND IMPRESSIONS}

To test the seventh hypothesis, an equality constraint was added to the baseline model. Hypothesis 7 suggested that the direct effect of second impressions formed at the beginning of the interview on final impressions of employability is significantly stronger than the direct effect of the structured interview score on final ratings of employability. The results shown in Table 6 provide parameter estimates and fit statistics for a constrained model in which the two relationships discussed above in Hypothesis 7.

Insert Table 6 About Here

The overall fit statistics of the constrained model closely mirror those of the baseline model with the CFI and SRMR indicating good fit according to the guidance of $\mathrm{Hu}$ and Bentler (1999) and the RMSEA (.069) falling very near the .06 cutoff for acceptable fit. The significance test for Hypothesis 7 was conducted by utilizing a Satorra-Bentler scaled chi square difference test (Satorra, 2000) that is shown in Table 7 below. The results suggests that constraining the two relationships specified in Hypothesis 7 to be equal does not significantly decrease model fit as indicated by the non-significant Satorra-Bentler scaled chi square difference test. This suggests that the two relationships are not significantly different from one another. This outcome fails to support Hypothesis 7 as the relationship between second impression and final impressions is not significantly stronger than the relationship between interview score and final impressions. 
Insert Table 7 About Here

\subsection{THE EFFECT OF DECISION CONFIDENCE ON DECISION STABILITY}

The first set of moderation hypotheses proposed in this manuscript addresses the effect of decision confidence on decision stability throughout the selection process. As specified in Chapter 4, I made an initial attempt to analyze these hypotheses using moderated multilevel structural equation modeling. These analyses failed to converge with program warnings indicating the presence of empirical underidentificaiton in the model.

In response to this non-convergence, I performed a standard path analysis utilizing the Huber-White Sandwich Estimator (Huber 1967; White, 1980) to control for the between-level variance while testing the hypotheses at the within-level. Hypothesis 8a suggested that confidence in initial ratings formed after the career fair would positively moderate the relationship between initial impressions formed at the career fair and second impressions formed at the beginning of the interview. As shown in Table 8, the interaction term between initial impressions and confidence in initial impressions was a significant predictor of second impressions of employability $(B=.051, p<.01)$. The form of this interaction is shown in Figure 6 below with the relationship between initial impressions and second impressions becoming stronger as decision confidence increases. These pieces of evidence provide support for Hypothesis 8a given that the relationship is significant and in the hypothesized direction. This finding, combined with the lack of support for Hypothesis 1, suggests that initial impressions 
only impact second impression of employability after considering an individual's confidence in that impression.

Insert Table 8 About Here

Insert Figure 6 About Here

In addition, Hypothesis $8 \mathrm{~b}$ suggested that confidence in second impressions of employability formed at the beginning of the interview would positively moderate the relationship between second impressions of employability and the structured interview score. As shown in Table 9, this hypothesis was not supported as the interaction term between second impressions and confidence in second impressions was not significant $(B=.007$, n.s. $)$. Likewise, Hypothesis 8c, which suggested that confidence in second impressions of employability would positively moderate the relationship between second impression and final impressions, was not supported $(B=.010, n . s$.$) . Results of this analysis are reported in Table 10$ below. In total, the results of the analyses for Hypotheses $8 \mathrm{~b}$ and $8 \mathrm{c}$ suggest that confidence in second impressions formed at the beginning of the interview do not modify the previously supported relationships between second impressions and both structured interview scores and final impressions of employability. 
Insert Table 9 About Here

Insert Table 10 About Here

\subsection{THE EFFECT OF INCENTIVE COMPENSATION ON DECISION STABILITY}

The final set of analyses addressed the relationships proposed in Hypotheses 9a, 9b, and $9 \mathrm{c}$ addressing the effect of incentive compensation on decision stability throughout the interview process. As discussed above, this analysis was conducted by performing a multiple group analysis within multilevel structural equation modeling in which significant differences were sought between experimental conditions (e.g. incentive and fixed compensation) regarding the stability of employment decisions over time. First, a baseline analysis was conducted by including the compensation conditions as grouping variables. The results of this analysis are included in Table 11 below and provide separate parameter estimates for both experimental condition as well as baseline fit statistics for performing Satorra-Bentler scaled chi square difference tests. Second, a fully constrained model was tested in which the propositions made in Hypotheses 9a, 9b, and 9c were all included in the model. The results of this analysis are included Table 12 below. This analysis resulted in a non-significant change in chi square as shown in the results of the Satorra-Bentler scaled chi square difference test show in Table 13. This indicates that a model in which the linkages between initial impressions and second 
impressions, second impressions and interview scores, and second impressions and final impressions are all moderated by incentive compensation does not provide adequate fit for the data. However, it is important to explore the separate linkages proposed in Hypotheses 9a, 9b, and $9 \mathrm{c}$ to fully explore the moderating effect of incentive compensation on decision stability.

Insert Table 11 About Here

Insert Table 12 About Here

Insert Table 13 About Here

Third, additional steps were taken to individually test each linkage for statistical significance. Hypothesis 9a suggested that incentive compensation would positively moderate the relationship between initial impressions of employability formed at the career fair and second impressions of employability. The results of this MSEM analysis are reported in Table 14 below and compared to the baseline model displayed in Table 11. The statistical test for group differences is reported in Table 15 in the form of a Satorra-Bentler scaled chi square difference test. The non-significant results of the test suggest that the strength of the relationship between 
initial impressions formed during the career fair and second impressions formed at the beginning of the interview does not vary as a function of compensation condition.

Insert Table 14 About Here

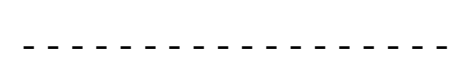

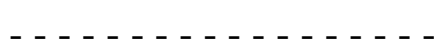

Insert Table 15 About Here

Additionally, Hypothesis 9b suggested that incentive compensation would moderate the relationship between second impressions formed at the beginning of the interview and structured interview scores. Results of the MSEM analysis and Satorra-Bentler scaled chi square difference test are reported in Tables 16 and 17 below. As shown in Table 17, the statistical test for group differences was non-significant indicating that compensation condition did not significantly impact the relationship between second impressions of employability formed at the beginning of the career fair and structured interview scores.

Insert Table 16 About Here 
Insert Table 17 About Here

Finally, Hypothesis 9c suggested that compensation condition would moderate the relationship between second impressions of employability and final impressions of employability. As conducted above, MSEM and Satorra-Bentler scaled chi square difference tests were performed to assess group differences and the results are reported in Tables 18 and 19 below. Results of these analyses mirror the results reported above indicating that there were no group differences between compensation conditions for the relationship between second impressions formed at the beginning of the interview and final impressions of employability. In total, these analyses suggest that incentive compensation did not affect the stability of employability ratings at any point throughout the interview process.

Insert Table 18 About Here

Insert Table 19 About Here 


\subsection{SUPPLEMENTAL ANALYSES}

In response to the above results, several supporting analyses were conducted to further explore the hypothesized phenomena. The first issue to be addressed in this section is the nonsignificant result for Hypothesis 7. Given that this hypothesis was actually enveloped in a larger mediated model (see Figure 5), the Satorra-Bentler scaled chi square difference test does not necessarily provide a true picture of the total effect of second impressions on final impressions of employability.

To further explore this relationship, I utilized several indices to provide further insight into the true magnitude of the indirect effect. The results of these analyses are reported in Table 20 below. First, the index of mediation (MacKinnon, 2008; Preacher \& Hayes, 2008) suggests that final impressions of employability increase .197 standard deviations for every one standard deviation increase in second impressions of employability indirectly via interview scores. This measure provides a standardized estimate of the indirect effect and is statistically significant ( $a b_{s x}$ $=0.197 ; p<.05 ; 95 \% \mathrm{CI}[.129, .259])$. Second, the measure of shared effects over simple effects (SOS; Lindenberger \& Pötter 1998) describes the proportion of variance in final impressions of employability that is explained by second impressions of employability and shared with interview scores. This test was significant $(\mathrm{SOS}=.588 ; p<.05 ; 95 \% \mathrm{CI}[.427, .708])$ and suggests that a significant amount of variance that is shared by second impressions of employability and interview scores is predictive of final impressions of employability. Third, the index of variance explained (MacKinnon, 2008) identifies the proportion of variance in final impressions of employability that can be attributed to both second impressions of employability

and interview scores but can be attributed to neither alone. The significant result of this test $\left(\mathrm{R}^{2}=\right.$ $0.299 ; p<.05 ; 95 \%$ CI $[.199 ; .386])$ suggest that the indirect effect of second impressions of 
employability on final impressions of employability transferred through interview scores explains $29.9 \%$ of the variance in final impressions of employability after removing all direct effects from second impressions of employability and interview scores. Finally, Preacher and Kelley (2011) proposed two new methods that are suggested to be more precise when describing the magnitude of the indirect effect. The standardized residual-based index of mediation, based on the work of Berry and Mielke (2002), describes the extent to which interview scores are explained by second impressions of employability and that variance in final impressions of employability that is jointly explained by second impressions of employability and interview scores. Results of this analysis provide support for the integrity of the indirect effect $(\Gamma=.199 ; \mathrm{p}$ $<.05 ; 95 \%$ CI $[.134, .267])$. In addition, Preacher and Kelly (2011) also advocated the consideration of the proportion of the maximum possible direct effect, which describes the magnitude of the indirect effect of second impressions of employability on final impressions of employability relative to the maximum possible indirect effect. This accounts for the maximum attainable value of the indirect effect conditional on sample variance and the magnitudes of other relationships in the model. This analysis also supported the integrity of the indirect effect $\left(\kappa^{2}\right.$ $=.250 ; \mathrm{p}<.05 ; 95 \% \mathrm{CI}[.167, .317])$. These analyses of explained variance provide interesting insight as to the true relationship between second impressions and final impressions of employability by highlighting that a sizeable portion of the total effect is due to the indirect effect of second impressions transferred through interview scores. This additional analysis provides interesting implications for future research as discussed later in Chapter 6. 


\section{Insert Table 20 About Here}

The second issue to address in this section is the failure to support the hypotheses addressing the effect of incentive compensation on employability rating stability (Hypotheses 9a, $9 \mathrm{~b}$, and 9c). In response to these non-significant results, I conducted a series of manipulation checks utilizing data collected before and after the simulation to explore the psychological effect of the compensation manipulation. Felt accountability was measured using an 8-item scaled developed by Hall, Frink, Ferris, Hochwarter, Kacmar, and Bowen (2003; Pre $\alpha=.702$; Post $\alpha$ $=.881$ ) and assessed an individual's perception that an experienced work demand requires explanation for potential external evaluation (Breaux, Munyon, Hochwarter, \& Ferris, 2009). Felt accountability was included as a manipulation check because it should provide some insight as to whether or not the recruiters in the incentive condition felt as though the performance management process held them more accountable than those in the fixed compensation condition. Additionally, impression management was measured using an 8-item scale developed by Wayne and Ferris (1990; Pre $\alpha=.778$; Post $\alpha=.781)$ that was included to determine whether or not the recruiters in the incentive compensation condition would engage in more impression management than those in the fixed compensation condition given the performance reviews and variable pay system used in the incentive condition. Finally, I measured identified regulation (Pre $\alpha=.614$; Post $\alpha=.783$ ), external regulation (Pre $\alpha=.750 ;$ Post $\alpha=.579$ ), and intrinsic motivation (Pre $\alpha=.826$; Post $\alpha=.847$ ) using subscales from Tremblay, Blanchard, Taylor, Pelletier, and Villeneuve's (2009) Work Extrinsic and Intrinsic motivation scale. These items 
were designed to assess the impact of the compensation manipulation on both extrinsic and intrinsic motivational structures among applicants. This variety of manipulation check scales should provide a well-rounded view of the motivational characteristics of each experimental group.

As discussed briefly above, recruiters in both compensation conditions completed these manipulation check scales at the beginning of the simulation after they were given details regarding their compensation condition and then again at the end of the simulation after completing all of their interviews. To assess the strength of the manipulation by conducting a ttest to assess group mean differences between conditions. Results of these analyses are reported in Table 21 below.

Insert Table 21 About Here

As show in the analyses above, the means for felt accountability when measured after the administration of the manipulation but before the simulation began were significantly different with the individuals in the incentive compensation condition reporting higher average levels of felt accountability $(\bar{x}=42.29)$ than those in the fixed compensation condition $(\bar{x}=36.43)$, which provides support for the integrity of the manipulation. However, there were no significant differences between groups for pre-simulation measures of impression management, identified regulation, external regulation, or intrinsic motivation. When measured after the simulation, none of the manipulation check scales (felt accountability, impression management, identified regulation, external regulation, or intrinsic motivation) showed significant differences between 
groups. In total, this evidence suggests that the manipulation did not make a significant difference in the psychological mindset of participants in each condition. This finding has important implications for future discussion and research that will be included later in this manuscript. 


\section{CHAPTER 6: DISCUSSION}

\subsection{MAJOR FINDINGS \\ 6.1.1 The Base Model: Employability Rating Stability}

One of the primary assertions of this manuscript is that employability ratings are formed early in the interview process and remain relatively stable over time. This assertion was posited within the first six hypotheses as represented previously in Figure 5. The results of this study provided partial support for this assertion. It was first proposed that impressions formed at a career fair would impact subsequent impressions during the interview process. This assertion was based on the gist extraction and fuzzy processing preference proposed by fuzzy trace theory (Brainerd \& Reyna, 1990) as well as the literature addressing thin slices of behavior (Ambady \& Rosenthal, 1992), but was not supported by the data. This suggests that a recruiter's initial impressions formed at a career fair do not affect subsequent decision processes without considering boundary conditions of the relationship. However, another assertion of this manuscript suggested that second impressions formed in the first few minutes of an interview would strongly impact both interview scores and final impressions of employability. This assertion was supported by the data as well as the thin slices of behavior literature (Ambady and Rosenthal, 1992) and the dual processing perspective (e.g. Schneider \& Schiffrin, 1977). Finally, this study also found support for the relationship between interview scores and final impressions of employability. In total, these findings suggest that interactions during career fairs do not significantly impact employment decisions, but impressions of employability appear to be relatively stable after the first few minutes of the interview. 


\subsubsection{Relative Importance: Second Impressions vs. Interview Scores}

Another major assertion of this manuscript suggested that initial impressions formed at the beginning of the interview would be significantly stronger than those formed during the remainder of the interview. Statistical significance testing failed to support this assertion in that there was not a significant difference between the effect of initial impressions formed at the beginning of the interview and the effect of interview scores when predicting final impressions of employability. Given this non-significant effect, an additional analysis was performed to examine the variance explained by the total effect of initial impressions and the direct effect of interview scores when predicting final impressions of employability. This analysis showed that the indirect effect of second impressions of employability on final impressions of employability transferred through interview scores, which was not considered in the MSEM analysis, is significant when considering the total effect of second impressions of employability. In total, this evidence suggests that initial impressions formed at the beginning of the interview make a definitive impact on final impressions of employability. This finding provides additional support to the empirical review of the thin slicing literature conducted earlier and the fuzzy processing preference proposed by Fuzzy Trace Theory (Brainerd \& Reyna, 1990).

\subsubsection{Decision Confidence and Employability Rating Stability}

To further explore the stability of employability ratings over time, two moderators were proposed to strengthen the relationship between ratings over time. The first moderating mechanism proposed to strengthen the relationships in the base model was decision confidence. This set of hypotheses proposed that an individual's confidence in a given decision would strengthen the relationship between the outcome of that decision and subsequent decisions. This 
assertion was supported by the literature addressing self-efficacy, which suggests that individuals that are more confident in their abilities achieve higher levels of performance (e.g. Judge \& Bono, 2001; Stajkovic \& Luthans, 1998). In addition, research addressing cognitive anchoring and confirmatory biases suggests that confidence could increase the salience of initial impressions thereby increasing decision stability (e.g. Schmitt, 1976; Simmons \& Nelson, 2006). Analytical results suggest that decision confidence moderates the linkage between initial impressions formed at the career fair and second impressions formed at the beginning of the interview. However, decision confidence does not appear to impact the relationship between either second impressions and structured interview score or second impressions and final impressions of employability. When combined with earlier findings from the base model, this suggests that initial impressions only impact second impressions after considering the effect of decision confidence. In addition, Figure 6 shows that the form of the relationship is in the hypothesized direction as the relationship between initial impressions and second impressions was strongest under high levels of decision confidence.

\subsubsection{Incentive Compensation and Employability Rating Stability}

The final major assertion included in this manuscript suggested that recruiters that were offered incentive compensation would provide more stable ratings of employability than those that were provided with fixed compensation. This assertion was supported by the extensive literature addressing incentive compensation (e.g. Jenkins et al., 1998; Stajkovic \& Luthans, 1997) as well as numerous theories of motivation (e.g. Reinforcement Theory, Skinner, 1938; Expectancy Theory, Vroom, 1964; Equity Theory, Adams, 1965; Goal Setting Theory, Locke, 1968). Despite this past empirical and theoretical support, the data failed to support this assertion through an omnibus test assessing the effect of incentive compensation on decision stability over 
time as well as individual analyses for the linkages proposed in Hypotheses 9a, 9b, and 9c. However, additional analyses were conducted to assess the psychological effect of the manipulation. These results suggested that the compensation manipulation did not significantly change felt accountability, impression management, identified regulation, external regulation, or intrinsic motivation during the simulation. As a result, it appears that the manipulation did not have the intended effect and that the results addressing the effect of incentive compensation on employability rating stability may not provide an accurate representation of the true relationship.

\subsection{CONTRIBUTIONS OF THE DISSERTATION}

Overall, there are four general contributions of this research that impact both research and practice. First, it appears that initial impressions matter in an interview context. This finding helps to clarify earlier work that provided somewhat conflicting evidence regarding the impact of initial interactions on final impressions of employability (e.g. Barrick et al., 2010; Chapman \& Zweig, 2005; Kinicki et al., 1990). In addition, it answers a recent call from Levashina and colleagues (2014) suggesting that additional work is needed to explore the true effect of rapportbuilding on the hiring process. This manuscript provides further support for the impact of initial impressions on final impressions of employability by showing the predictive validity of initial impressions (Hypothesis 2). Despite the fact that the hypothesis proposing that initial impressions formed at the beginning of the interview would be significantly stronger than interview interactions was not supported (Hypothesis 7), supplemental analyses further supported the importance of second impressions of employability by suggesting that the indirect effect transferred through interview scores provides significantly more explanatory power for second impressions of employability. All of this evidence suggests that initial impressions have important implications for both future research and practice in the context of employee selection. 
Second, the findings from this study suggest that thin slices of behavior are highly predictive in an interview context (Hypotheses 2,3,5, and 8a). The purpose of this line of inquiry was to bring a theoretical perspective to existing research addressing initial interactions in employment interviews (e.g. fuzzy trace theory) and to provide additional empirical evidence on this subject. The theoretical and empirical evidence provided in this manuscript helps to explain prior findings addressing initial impressions (e.g. Barrick et al., 2010; Chapman \& Zweig, 2005; Kinicki et al., 1990), while also providing a prescriptive theory for practicing managers assessing recruiter training and staffing processes. In addition, it provides empirical support for the literature addressing thin slices of behavior as well as a theoretical perspective to support the existing empirical evidence in this area.

Third, it appears that decision confidence may be a key factor for storage and future processing of information received in thin slices of behavior. Results for the hypotheses suggested two primary implications for future research. First, it appears that decision confidence may not impact future decision processes when information is presented in short time intervals. Results of this study showed that confidence in initial impressions formed at the beginning of the interview did not moderate the relationship between initial impressions formed at the beginning of the interview and either interview scores or final impressions of employability. This result does not support the assertion that decision confidence produces cognitive anchoring as reported in the existing literature (Bazerman, 1990; Tversky \& Kahneman, 1974). Second, it appears that decision confidence may impact future decision processes when a substantive temporal gap is present between information processing sessions. Results of this study suggest that confidence in impressions formed at the career fair positively moderates the relationship between impressions formed at the career fair and impressions formed at the beginning of the interview. This finding 
supports the fuzzy trace theory (Brainerd \& Reyna, 1990) principle of gist extraction and the idea that System 1 from the dual processing perspective can simplify information for long-term storage (e.g.Stanovich, 1999). In all, these findings suggest that there may be situational factors that impact the transfer of information from active processing to long term storage for future use.

Finally, this research provides additional insight regarding the effect of incentive compensation on non-executive decision makers. As discussed earlier, a wealth of research has addressed the impact of incentive compensation on executive decision making (e.g. GomezMejia \& Wiseman, 1997; Martin et al., 2013; Pepper \& Gore, in press). On the other hand, nonexecutive decision makers make daily decisions that impact organizational performance and competitive position (Becker \& Gerhart, 1996; Floyd \& Wooldridge, 1997) with very little research addressing this phenomenon. Analyses contained within this study suggest that incentive compensation may not make a substantive impact on non-executive decision making (Hypotheses 9a, 9b and 9c). However, manipulation checks assessing the psychological impact of the compensation manipulation revealed that the manipulation did not have its intended effect. As a result, there are two key points that should be considered regarding this finding. First, incentive compensation may not impact decision processes of non-decision makers. Second, additional research is needed to further clarify this relationship as the non-finding is likely due to the implementation of the manipulation as shown by the non-significant manipulation check analyses. 


\subsection{STRENGTHS AND LIMITATIONS OF THE RESEARCH DESIGN}

\subsubsection{Strengths}

Given the nature of this sample (a student sample collected during an interview simulation), there are numerous strengths and weaknesses of the design. The primary strength of this design is the utilization of longitudinal, multi-source data collected using a variety of different survey instruments. Survey instruments included interviews, Likert-type paper surveys, Likert-type electronic surveys, and objective report surveys (e.g. biodata) that were provided by both recruiters and applicants. This design helps to prevent single source and self-report biases while also ensuring that the hypothesized linkages for temporal precedence are confirmed with statistical analyses. In addition, the multi-source, longitudinal data helps to address the repeated testing (different survey types are used for repeated measures), confounding (longitudinal data helps to establish temporal precedence), and instrumentation (use of multi-source data) threats to internal validity.

Another strength of this design is the use of a realistic simulation based on the hiring process of a Fortune 50 organization. Consultative meetings were conducted with representatives from the corporate sponsor to ensure that the processes, timing, interview questions, and decision processes closely resembled those used by their Fortune 50 organizations. The only changes made to the processes utilized by the sponsoring organization were the implementation of the initial impression scales that were necessary for this study. By collaborating with this Fortune 50 organization, I was able to address threats to external generalizability given that this process closely mimics real hiring procedures. 
To complement the above strengths, another benefit of using this particular design was the tight experimental control over temporal sequencing in the longitudinal design. It would be difficult to ensure that recruiters observed the precise sequencing necessary for this study if the interviews were conducted at various locations by recruiters in the field. This aspect of the design helped to minimize the confounding, mortality, and history (experimental control of the time between repeated measures) threats to internal validity.

Finally, the use of trained interviewers with working experience in Human Resource Management should be considered a strength of this design. All participants completed a 16 week course addressing talent acquisition and completed a 3 month internship in the field of Human Resource Management. In addition, each recruiter had previously participated in this interview simulation by completing between 7 and 9 interviews each. These steps help to address external generalizability given that the recruiters all have experience in the field of Human Resource Management and that they all conducted structured interviews before participating in this experiment.

\subsubsection{Limitations}

Despite the above strengths, there are also several weaknesses of this design that should be salient to the reader when drawing conclusions from this study. The primary limitation of this study is the use of simulated mock interviews. While the above strengths provide mundane realism in that the process closely mirrors that of a Fortune 50 organization, the simulated nature of the interviews threatens experimental realism in that participants may not be fully invested in the interview process given that there is no actual job to be had. It is important to note that metaanalytic evidence suggests that results differ very little when using lab-based data rather than 
field-based data with lab-based data generally providing slightly weaker effect sizes (Anderson, Lindsay, \& Bushman, 1999; Colquitt, 2014). In addition, it is also important to note that these data would be very difficult to collect in the field given the dedication necessary on the part of the recruiter and the confidentiality and logistical issues involved with obtaining informed consent from each applicant. Despite this evidence that lab-based data and field-based data may provide similar conclusions, the lab-based nature of this study creates a threat to external generalizability.

A second limitation of this design is the use of student participants. Despite decades of criticism on the topic of student samples, research suggests that student samples can produce highly valid results as long as situational factors are considered (e.g. Dipboye \& Flanagan, 1979; Ilgen, 1986). For example, it would not be advisable to use students in a study that attempts to uncover the decision making processes of CEOs. However, this study utilizes students that are actual members of the population of interest as the students participating as applicants were all upper-level students pursuing internships and jobs. In addition, all students that participated as interviewers had experience in the field of Human Resource Management, experience as an interviewer, and interview training equivalent to a new graduate of a master's program in Human Resource Management. For these reasons, it appears that the use of students for this study should provide appropriate conclusions that are generalizable to the population of interest. In any case, the use of students could possibly threaten the external generalizability of this study.

The third primary limitation of this study is the broad job description used for the basis of the simulation. The job description is included in Appendix $G$ of this manuscript. The lack of specificity in the job description allowed students from across disciplines (management, marketing, finance, accounting, management information systems, economics, hospitality and 
tourism, and general business) to apply for the same management development program. The rotational nature of the program gave an applicant the opportunity to focus discussion on topics dealing with his or her own major while also discussing his or her ability to be flexible in other functional areas. As a result of the broad job description, the interview questions (shown in Appendix A) were also somewhat broad and not task specific so as to allow the applicant to discuss examples related to his or her area of specialization. In this way, utilizing a broad job description and broad interview questions was the best option for this study given the diverse background of those that comprise the sample. That being said, this lack of specificity could impact external generalizability in that most positions would include job descriptions that are more tailored to the applicant's skills and qualifications.

Another limitation of this study is the failed manipulation checks for the compensation manipulation. The manipulation was implemented via the compensation scripts displayed in Appendix D and the employment agreements in Appendices E and F. These documents clearly outlined the compensation structure in multiple locations to ensure that recruiters were fully aware of their own incentive condition. In addition, a non-disclosure agreement was included in the employment agreement to prevent information spillover between conditions. As discussed above in Section 5.6 (Supplemental Analyses), recruiters in the incentive compensation generally did not show higher levels of felt accountability, impression management, identified regulation, external regulation, or intrinsic motivation as compared to those in the fixed compensation condition. Informal interactions with recruiters when distributing gift cards indicated that the failure likely occurred with regards to the strength of the manipulation as most of the recruiters did not remember their own compensation structure. Given these failed manipulation checks, the 
internal validity of the above conclusions regarding the effect of incentive compensation on recruiter decision processes appears to be in question.

A fifth major limitation of this study was the lack of realism at the career fair. At the simulated career fair, applicants were given the opportunity to meet their recruiter and establish rapport through informal discussion. The experimental administrator then provided an overview of the job itself and the basics of the hiring process used in the simulation. At the end of the event, applicants and interviewers were given more time to speak informally and schedule an interview. This design allowed for random assignment which should increase internal validity. However, there is also a threat to external validity created by assigning recruiters to applicants. At a normal career fair, applicants and recruiters have the opportunity to "shop around" to look for the best fit between applicant and organization. In this simulation, recruiters were required to interview all applicants regardless of their qualifications or perceived fit with the organization. Likewise, applicants were required to interview with the hypothetical organizational regardless of their perceptions of fit with the organization. As a result, readers should understand that these inconsistencies between the simulated career fair and actual career fairs may increase the internal validity of the statistical conclusions, but limit the experimental realism and external generalizability of the study.

The final major limitation of this study is the measurement of initial impressions after the career fair and during the interview. Recruiters completed the five-item scales for both employability and confidence (shown in Appendix B) to assess their perceptions of the applicant's suitability for employment after the career fair and at the beginning of the interview. It is unlikely that field recruiters would be asked to recall and record these initial impressions when conducting actual interviews. As a result, these scales could potentially create cognitive 
anchors for the recruiters by making their initial impressions more salient and impactful when completing the interview process. This effect could threaten internal validity via repeated testing and instrumentality and should be considered when interpreting the results of this study.

\subsection{IMPLICATIONS FOR RESEARCH}

The primary implication of this research is that initial impressions strongly predict outcomes in an interview context. Fuzzy trace theory (Brainerd \& Reyna, 1990) and the thin slicing perspective (Ambady \& Rosenthal, 1992) support this finding theoretically. The purpose of the hypotheses that produced this finding was to reenergize a discussion in the literature that addresses the impact of initial impressions on final impressions of employability. This finding produces several important implications for future research.

First, this finding shows the need to provide further statistical testing addressing the shared variance between initial impressions and impressions formed during the remainder of the interview. While Hypothesis 7, which suggested that initial impressions would be significantly stronger than interview scores when predicting final impressions of employability, was not supported, additional analyses revealed that a substantive portion of the variance normally attributed to impressions formed during the interview should actually be attributed to initial impressions. Second, this finding suggests that we need to know more about the information that is actually being captured in the initial thin slice interaction. It is possible that the information collected in these initial interactions is task relevant and therefore useful for valid selection. However, it is also very possible that these initial impressions capture biases and discriminatory information. Third, information presented in Figures 8 and 9 suggest that there are individual differences in rating stability across recruiters. Each numbered graph represents a recruiter with 
each line tracking an individual applicant's employability rating from initial impressions formed at the career fair through final impressions of employability. These individual differences could provide interesting implications for research in that some recruiters may be more likely to utilize their initial impressions for decision making than others.

Insert Figure 8 About Here

Insert Figure 9 About Here

Another interesting implication that results from this research is the mixed evidence for the predictive validity of fuzzy trace theory in a selection context. Despite the relatively long existence of fuzzy trace theory (Brainerd \& Reyna, 1990), it does not appear that this theory has yet been applied to an organizational context. In support of the theory, it appears that fuzzy trace theory provides a useful theoretical perspective to the literature addressing thin slices of behavior. The fuzzy trace theory principles of the fuzzy to verbatim continuum and the fuzzy processing preference appear to be empirically supported by this study. Given this initial empirical support, it appears that fuzzy trace theory could be beneficial for complementing the relatively atheoretical literature addressing thin slices of behavior. However, these results provide mixed support for the third prong of fuzzy trace theory that posits the ability for gist extraction. The gist extraction component of the theory was tested with Hypotheses 1, which 
proposed that impressions formed at the career fair would impact subsequent impressions formed at the beginning of the interview, and Hypothesis 2, which postulated that second impressions formed at the beginning of the interview would subsequently impact final impressions of employability. The first hypothesis was not supported which suggests that individuals might not be able to collect and store gist information in long-term storage for future processing. However, the second hypothesis was supported which suggests that gist extraction may be useful only in situation where decisions are made soon after the thin slice. In all, this evidence suggests that fuzzy trace theory might be a useful perspective for conducting future organizational research.

Finally, these results provide support for the predictive validity of the thin slicing perspective in an organizational decision making context. While earlier research utilizing the thin slicing perspective has addressed topics such as assessing Big Five traits (Ames et al., 2010), teaching evaluations (Tom et al., 2010), and psychopathy levels (Fowler et al., 2009), very little research has assessed the predictive validity of thin slices of behavior in an organizational context. In addition, very little research (see Curhan \& Pentland, 2007 for work that informs this topic) has addressed the impact of information collected during thin slices of behavior on final decision outcomes. Results from Hypotheses 3 and 4 as well as the supplemental analyses provide support for the predictive validity of the thin slicing perspective in organizational decision making. This suggests that individual rely heavily on information that they receive in the first few minutes of the interview when making employment decisions. Building upon this finding, it appears that the thin slicing perspective (coupled with fuzzy trace theory as discussed above) may provide a useful framework for research addressing organizational decision making. 


\subsection{DIRECTIONS FOR FUTURE RESEARCH}

In response to the research implications listed above, there are several logical directions for future research based on the results of this study. Most importantly, there is now a clear need to assess the validity of the predictive power of initial impressions in the context of final impressions of employability. The key question in this area is as follows: "What is actually driving these initial impressions?" As noted above (and supported by the results from Hypotheses 3 and 4 as well as the supplemental analyses), initial impressions account for a large portion of the variance in final impressions of employability. As a result, future research should try to further understand the type and validity of information that is collected during these initial interactions. This investigation could address at least three key topics.

First, it is important to know whether the information collected during these initial interactions is task relevant. Research suggesting that initial interactions result in task relevant information would suggest that incorporating initial impressions into selection decisions could provide valid hiring decisions. However, research suggesting that this information is not task relevant would suggest that practitioners need to discourage the introduction of initial impressions into the decision making process. Second, researchers should assess the impact of utilizing initial impressions in decision making on the validity of job analyses. Organizations spend millions of dollars each year to perform job analyses that accurately describe the duties and responsibilities of the job itself. These job analyses are then used as a foundation for selection systems to increase the validity of the selection process (Wright \& Boswell, 2002). Given this investment, it is important to determine whether or not these initial impressions capture information that is relevant to the duties and responsibilities for the position that were identified in the job analysis. Third, it is important to assess whether or not these initial 
impressions introduce discriminatory biases into final selection decisions. The introduction of discriminatory biases, which would produce both legal and potentially performance implications, would suggest that practitioners should suppress the impact of initial impressions on final selection decisions. In all, developing a further understanding of the source and content of initial impressions could dramatically improve our understanding of the employee selection process.

The second primary research implication results from the implication described above. Some evidence suggests that initial impressions can introduce bias and discrimination into the selection process (e.g. Huffcutt, 2011; Richeson \& Shelton, 2005). As a result, future research should explore the use of a variety of tools for reducing or eliminating the bias that is inherent in initial impressions. The first option is to implement recruiter training procedures that are designed to reduce bias in initial impressions. As noted in past research (e.g. Connerly, 1997), recruiter training procedures can help to reduce bias in interview scores. A natural extension of this research is to examine the impact of recruiter training on biases in initial impressions. It is very possible that increasing awareness on biases in initial impressions could make a substantive difference in outcomes as past research has shown that increasing information salience can modify decision processes (e.g. Stasser, 1992). Another possibility is to explore the use of more detailed behaviorally anchored rating scales (BARS; Jacobs et al., 1980). This inquiry could manipulate the specificity of the BARS provided to recruiters to see if providing more guidance results in decreased reliance on initial impressions. In addition, future research could assess the utility of using multiple recruiters in selection interviews. It is possible that multiple recruiters could produce more valid results that are less impacted by initial impressions by providing multiple perspectives on each applicant. Finally, evidence suggesting that initial impressions introduce bias into the selection process would encourage further research addressing 
supplemental assessment methods for employee selection. Despite numerous calls in the literature for supplemental assessments, it appears that numerous organizations still rely solely on interviews (Highhouse, 2008). Future research addressing the use of supplemental assessment methods for reducing bias related to initial impressions could create a more compelling argument for swaying practicing managers to engage in evidence-based management.

The third primary direction for future research involves statistically analyzing the total effect of initial impressions on final impressions of employability as compared to the effect of interview scores. As documented in the supplemental analyses section, the indirect effect of second impressions of employability on final impressions of employability transferred through interview scores explains a significant amount of variance. However, it does not appear that a statistical method currently exists to assess the statistical difference between the total effect of initial impressions and the direct effect of interview scores. It would be beneficial to address this issue in future research by applying new statistical tools to assess the statistical significance of the difference in predictive power between the total effect of initial impressions and the direct effect of interview scores.

The fourth potentially fruitful direction for future research involves additional inquiry addressing the impact of compensation on recruiter decision making processes. As shown in the supplemental analyses section (Table 20), recruiters in the incentive compensation condition did not show significant differences in pre-simulation or post-simulation measures of impression management, identified regulation, external regulation, or intrinsic motivation or the postsimulation measure of felt-accountability. This suggests that the manipulation did not have its intended effect therefore leading to the non-significant results for Hypotheses 9a, 9b, and 9c. Past research provides extensive theoretical support for these potential relationships by applying 
well-supported theories such as goal-setting theory (Locke, 1968), expectancy theory (Vroom, 1964), and equity theory (Adams, 1965). In addition, a wealth of empirical evidence suggests that compensation can be used as a motivational tool to concentrate effort and increase performance (e.g. Colquitt et al., 2001; Eisenberger \& Cameron, 1996; Hollenbeck \& Klein, 1987). Finally, there appears to be very little research addressing the impact of incentive compensation on the decision processes of non-executive employees. As a result, it appears that future research addressing the effect of incentive compensation on recruiter decision stability could address a gap in the literature while also providing useful implications for practitioners.

The fifth proposed direction for future research involves exploring the impact of intrinsic motivation on decision stability. One explanation for the non-significant results for the incentive compensation hypotheses and manipulation checks is that Deci and Ryan's (1985) cognitive evaluation theory (CET) provides more accurate predictions than the motivational theories discussed earlier. While meta-analytic evidence suggests that extrinsic rewards do not significantly impact intrinsic motivation (e.g. Deci et al., 1999; Eisenberger \& Cameron, 1996), intrinsic motivation itself may prove to be a useful explanatory mechanism when considering decision stability in selection decisions. This idea is based on the original work of Lepper and colleagues (1973) suggesting that individuals expend more effort on tasks that they find interesting and inherently motivating. As a result, intrinsic motivation could increase decision stability due to increased focus and attention during early phases of the selection process. This research would provide additional support for the basic foundation of CET while also providing practitioners with a measurable individual difference that impacts decision processes during employee selection. 
A sixth and final proposed direction for future research is exploring the impact of initial impressions in unstructured interviews and the subsequent validity of those impressions. As noted by Highhouse (2008), many organizations still stubbornly rely on unstructured interviews alone for employee selection. Meta-analytic results show that unstructured interviews perform quite poorly for predicting objective performance outcomes (Vinchur, Schippmann, Switzer, \& Roth, 1998). In addition, we know that structured interviews are superior to unstructured interviews in that they produce more valid results and less biased conclusions (Dipboye, 1994; Macan, 2009). Given this widely accepted finding, it is very possible that initial impressions in unstructured interviews are even more important than in structured interviews. Future research could address this question by reexamining Hypothesis 7, which suggested that initial impressions would be significantly stronger than interview scores, in the context of unstructured interviews. This area of research could provide a more compelling argument for the use of structured interviews and other assessments among practitioners while also addressing a gap in the current research assessing the impact of initial impressions on final decisions when the decision making process lacks structure.

\subsection{IMPLICATIONS FOR PRACTICE}

To complement the research implications described above, there are also several implications for practice that result from these findings. The primary implication for practitioners is that initial impressions during the interview process have a strong impact on final impressions of employability (as supported with Hypotheses 2, 3, 4 and 6). In response, practitioners should consider adding complementary selection tool such as surveys or job simulations to ensure that task-relevant information is used for selection purposes. Another option is to limit rapportbuilding that is a primary source of these initial impressions. However, while existing research 
does suggest that limiting rapport-building can increase the validity of selection interviews (e.g. Barrick et al., 2010), this may not be a highly practical recommendation given the awkward nature of commencing an interview without even exchanging pleasantries. A third option would be to remove the recruiter from the final decision-making process and instead rely on their notes and scores for making selection decisions. This could remove some bias from the process by using notes provided in a journalistic fashion by the recruiter and somewhat objective BARS to make decisions. In any case, it appears that practitioners should be wary of the effects of initial impressions on interview scores until researchers further examine the task-relatedness of these impressions. Using the strategies above should help to improve the accuracy of the selection process while also reducing bias.

Another important implication for practitioners is the finding that impressions formed at career fairs do not appear to impact employment decisions (Hypothesis 1). Anecdotal evidence suggests that many companies are abandoning the traditional paradigm of attending career fairs during the staffing process due to the high cost and low return on investment of sending recruiters from career fair to career fair. This research supports this decision by suggesting that impressions formed at the career fair do not seem to impact future decision processes without considering decision confidence as a boundary condition. However, it is important to note that attending career fairs can be useful for employer branding and applicant attraction purposes and may provide benefits beyond the selection process itself. In any case, interactions occurring at the career fair do not appear to impact selection decisions when considered alone.

Despite the finding that initial impressions at the career fair alone do not impact subsequent decision processes, results did suggests that initial impressions formed at the career fair can impact future decision processes as decision confidence increases (Hypothesis 8a). This 
suggests that interactions at the career fair can inform the remainder of the selection process if recruiters are confident in those initial impressions. Given this finding, organizations wishing to incorporate career fair interactions into their selection decisions could consider sending more experienced recruiters that are more confident in their ability and subsequently more confident in their initial impressions. This relationship might be dependent upon the amount of information that the applicant provides during career fair interactions. However, incorporating these initial impressions into the decision process may not be the best course of action given that future research is needed to address the task-relatedness of these initial impressions. As a result, sending less confident recruiters to career fairs that are less likely to be confident in their initial impressions might be the best course of action for reducing bias and increasing selection accuracy.

\subsection{CONCLUSION}

This dissertation focused on employability rating stability throughout the selection process by examining career fair interactions, initial impressions at the beginning of the interview, and the interview itself in an attempt to find the true source of hiring recommendation variation. In addition, this dissertation proposed that decision confidence and incentive compensation would increase employability rating stability over time. Using a sample of 229 applicants and 28 recruiters, it was found that career fair interactions have little effect on subsequent decision processes. However, initial impressions formed at the beginning of the interview and the interview score itself were both strong predictors of final ratings of employability. Incentive compensation did not increase decision stability across time, but manipulation checks failed to support the integrity of the compensation manipulation. Finally, decision confidence only impacted decision stability for the relationship between initial 
impressions formed at the career fair and initial impressions formed at the beginning of the interview. These results, displayed graphically in Figure 7 and listed in Table 22 below, display the importance of initial impressions in an interview context and strongly encourage future research addressing the validity of these initial impressions for selection purposes.

Insert Figure 7 About Here

Insert Table 22 About Here 
APPENDIX A

STRUCTURED INTERVIEW FORM

Recruiter \#

Applicant

Total BI Score

Open with some type of rapport builder: i.e., How are you? Tell me about yourself?

Tell me why you want to work for your company and what qualifications you can bring to the organization.

Notes:

\begin{tabular}{|c|c|c|c|c|c|c|c|c|c|c|c|c|}
\hline & \multicolumn{7}{|c|}{ Assessment } & \multicolumn{5}{|c|}{ Confidence } \\
\hline & \multicolumn{2}{|c|}{$\begin{array}{l}\text { Extremely } \\
\text { Low } \\
\end{array}$} & & & & \multicolumn{2}{|c|}{$\begin{array}{r}\text { Extremely } \\
\text { High } \\
\end{array}$} & \multicolumn{2}{|c|}{$\begin{array}{l}\text { Not } \\
\text { Confident } \\
\end{array}$} & & \multicolumn{2}{|c|}{$\begin{array}{r}\text { Highly } \\
\text { Confident }\end{array}$} \\
\hline Ability to do the job & 1 & 2 & 3 & 4 & 5 & 6 & 7 & 1 & 2 & 3 & 4 & 5 \\
\hline Potential for quality output & 1 & 2 & 3 & 4 & 5 & 6 & 7 & 1 & 2 & 3 & 4 & 5 \\
\hline Potential for quantity output & 1 & 2 & 3 & 4 & 5 & 6 & 7 & 1 & 2 & 3 & 4 & 5 \\
\hline Will get along with others & 1 & 2 & 3 & 4 & 5 & 6 & 7 & 1 & 2 & 3 & 4 & 5 \\
\hline Has potential for promotion & 1 & 2 & 3 & 4 & 5 & 6 & 7 & 1 & 2 & 3 & 4 & 5 \\
\hline
\end{tabular}

Use the following scale as you analysis the responses from the applicant for the questions below.

\begin{tabular}{|l|l|}
\hline $\mathbf{1}=$ Poor & $\begin{array}{l}\text { gave no detail, answer presented no relevance to question, answer was vague, exhibited no } \\
\text { knowledge in answer }\end{array}$ \\
\hline $\mathbf{2}$ = Fair & $\begin{array}{l}\text { gave some detail, answer presented minimal relevance to question, answer was somewhat } \\
\text { clear, but still lacking, exhibited minimal knowledge }\end{array}$ \\
\hline $\mathbf{3}=$ Good & $\begin{array}{l}\text { gave specific details, answer was relevant in a general sense, answer contained few unclear } \\
\text { statements, exhibited basic KSAOs pertaining to position }\end{array}$ \\
\hline $\mathbf{4}=$ Excellent & $\begin{array}{l}\text { showed great depth of detail, answer was directly related to question, answer contained no } \\
\text { unclear statements, exhibited many specific KSAOs pertaining to position }\end{array}$ \\
\hline
\end{tabular}

\begin{tabular}{|l|l|}
\hline \multicolumn{1}{|c|}{ Questions } & Points \\
\hline 1. Tell me about a major accomplishment you have had, please be specific. & \\
\hline Notes: & \\
\hline
\end{tabular}


2. Give me an example of a good decision you have made recently. What alternatives did you consider and why did you choose the one alternative over the others?

Notes:

3. Tell me about a project you organized, coordinated, and executed.

Notes:

4. Tell me about a time you missed an important deadline. How did you remedy the situation? What would you do differently and why?

Notes:

5. Can you give a recent example of when you have been able to apply basic managerial principles in a professional or classroom setting? (Looking for professional lingo or terminology and actual practice)

Notes: 
6. Give an example of when you and your supervisor/professor had conflicting ideas. How did you handle the situation?

Notes:

7. Describe a situation in which you worked with a team that had conflicting interests. How did you use your interpersonal skills to resolve the conflict? Notes:

8. Is there any reason why you could not fulfill the job as defined in the job description?

Notes: 


\begin{tabular}{|l|l|}
\hline 9. What questions do you have for me? (This indicates their preparedness.) & \\
\hline Notes: & \\
\\
\\
\hline Miscellaneous Notes: & \\
\hline Notes: & \\
\end{tabular}




\section{APPENDIX B \\ MEASUREMENT SCALES}

Employability and Confidence Scales

Please rate the likelihood of this candidate to address the following issues to assess the employability of this candidate:

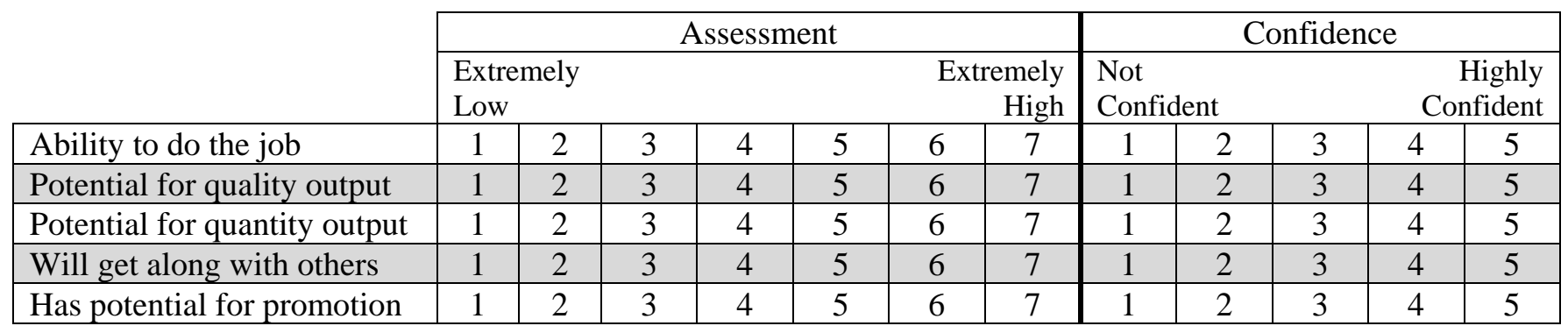

Please select your gender from the list below:

\begin{tabular}{|l|}
\hline Female \\
\hline Male \\
\hline
\end{tabular}

Please select your race from the list below:

\begin{tabular}{|l|}
\hline American Indian or Alaska Native \\
\hline Asian \\
\hline Black or African American \\
\hline Hispanic or Latino \\
\hline Native Hawaiian or Other Pacific Islander \\
\hline White \\
\hline Other \\
\hline
\end{tabular}

Please select the range below that represents your overall Grade Point Average:

\begin{tabular}{|l|}
\hline$<2.0$ \\
\hline 2.1 to 2.5 \\
\hline 2.6 to 3.0 \\
\hline 3.1 to 3.5 \\
\hline 3.6 to 4.0 \\
\hline
\end{tabular}


Please indicate your level of work experience:

Less than one year

One to three years

More than three years 


\section{APPENDIX C \\ INSTITUTIONAL REVIEW BOARD APPROVAL LETTER}

Approval Letter Expedited

To

From

Approval Period

Expiration Date

Subject Protocol

Protocol Number

Title
Joyce Heames

WVU Office of Research Integrity and Compliance

$08 / 24 / 2014$

$08 / 23 / 2015$

Approval Letter

1407352987

The temporal nature of employee selection processes

The above-referenced research study was reviewed by the West Virginia University Institutional Review Board IRB and was approved in accordance with 46 CFR 46.101b.

It has been determined that this study is of minimal risk and meets the criteria as defined by the expedited categorys listed below:

- APPROVE: The researchers have made the requested revisions to this Expedited Protocol (Category 7).

Documents for use in this study have been approved and validated and are available in the WVUkc system in the Notes and Attachments section of your protocol.

The Office of Research Integrity and Compliance is here to provide assistance to you from the initial submission of an IRB protocol to its approval and all subsequent activity. Please feel free to contact us by phone at 304.293.7073 with any question you may have. Thank you.

WVU Office of Research Integrity and Compliance

Date: $08 / 24 / 2014$

Signed: 


\title{
APPENDIX D \\ INCENTIVE COMPENSATION SCRIPTS
}

\author{
Fixed Compensation Script
}

Good afternoon everyone. As a sign of our appreciation, we would like to provide a twenty-five dollar gift card as a bonus to each of you upon the completion of this hiring process. You will each receive your gift card as soon as the process is completed.

\section{Incentive Compensation Script}

Good afternoon everyone. In recognition of your efforts here at (insert company name here), we would like to recognize your superior performance with monetary incentives. High performers in your region will each be awarded a one hundred dollar gift card at the end of this hiring process, depending upon evaluation of your performance. These awards will be based upon the results of a $360^{\circ}$ performance appraisal that includes feedback from your supervisor, peers, and applicants. The gift cards will be distributed upon the completion of this hiring process and the performance management appraisal process. 


\section{APPENDIX E \\ FIXED COMPENSATION CONDITION EMPLOYMENT AGREEMENT}

CORPORATE SPONSOR Interview Competition

SIMULATION ADMINISTRATOR NAME

SIMULATION Administrator

ADMINISTRATOR LOCATION

(555) 555-5555

ADMINISTRATOR@ADMINISTRATOR.EDU

Dear

We are pleased that you have accepted this employment offer to participate in the CORPORATE SPONSOR Interview Competition as a recruiter. Your duties will begin on September $2^{\text {nd }}, 2014$.

This letter confirms the terms and conditions of your employment.

1. Scope of Work - As a recruiter, you are responsible for attending the "career fairs," conducting interviews, participating in decision processes, and completing any and all surveys requested by the COMPETITION administrator.

2. Compensation - You will be compensated a flat rate of $\$ 10$ per hour for attending the organizational meeting, "career fairs," and decision processes. In addition, you will be paid $\$ 10$ for each interview that you conduct. Finally, you will also earn a $\$ 25$ bonus for successfully completing the interview simulation.

3. Non-Disclosure - By signing below, you agree to withhold any and all information regarding compensation, applicant characteristics, applicant qualifications, and interview interactions from simulation participants, non-participants, and fellow recruiters until such point that the COMPETITION administrator permits you to do so.

Name

Signature

Date

Note: Information in all caps will be modified to recognize the actual administrator and corporate sponsor as appropriate. 


\begin{abstract}
APPENDIX F
INCENTIVE COMPENSATION CONDITION EMPLOYMENT AGREEMENT
\end{abstract}

CORPORATE SPONSOR Interview Competition

SIMULATION ADMINISTRATOR NAME

SIMULATION Administrator

ADMINISTRATOR LOCATION

(555) 555-5555

ADMINISTRATOR@ADMINISTRATOR.EDU

Dear

We are pleased that you have accepted this employment offer to participate in the CORPORATE SPONSOR Interview Competition as a recruiter. Your duties will begin on September $2^{\text {nd }}, 2014$.

This letter confirms the terms and conditions of your employment.

1. Scope of Work - As a recruiter, you are responsible for attending the "career fairs," conducting interviews, participating in decision processes, and completing any and all surveys requested by the COMPETITION administrator.

2. Compensation - You will be compensated a flat rate of $\$ 10$ per hour for attending the organizational meeting, "career fairs," and decision processes. In addition, you will be paid $\$ 10$ for each interview that you conduct. Finally, you will also have the opportunity to earn a $\$ 100$ bonus, depending on evaluations of your performance.

3. Non-Disclosure - By signing below, you agree to withhold any and all information regarding compensation, applicant characteristics, applicant qualifications, and interview interactions from simulation participants, non-participants, and fellow recruiters until such point that the COMPETITION administrator permits you to do so.

Name

Signature

Date

Note: Information in all caps will be modified to recognize the actual administrator and corporate sponsor as appropriate. 


\section{APPENDIX G}

JOB DESCRIPTION

\section{MANAGEMENT DEVELOPMENT PROGRAM}

\section{POSITION SUMMARY}

The Management Development Program (MDP) is a structured training program that provides future managers with a working knowledge of the major functional responsibilities in the company. The year-long program is designed to place trainees in rotations of varying length in each of the eight major functional areas of the company. No rotation is less than four weeks, and the rotation in the trainee's preferred functional area is at least eight weeks.

A primary objective is to provide participants with a broad, yet detailed exposure to the entire company that helps prepare them both for their immediate responsibilities as well as more general management responsibilities in the longer term. At the same time, the program allows each functional area of the company to assess the potential "fit" between the trainees and the job requirements of the function.

\section{RESPONSIBILITIES}

While labeled a development program, and packed with classroom sessions to expose participants to the company and prepare them for positional assignments, trainees are also assigned specific functional assignments in each rotation to provide "on-the-job" training. And, given the company's rich history of the use of project teams, participants will be assigned to at least one project team during the rotation sequence and given specific assignments to be completed. Upon completion of the year-long program, offers of employment are extended to trainees based on their evaluated performance in the training sessions, project activities, and functional assignments. The range and type of offers extended generally depend on functional area need and the functional preferences of the trainees themselves. In most cases, trainees assume some level of management responsibility.

The functional areas included in the MDP rotation include Corporate Accounting, Financial Budgeting and Analysis, Marketing, Sales, Human Resources, Research and Development, Economic Forecasting, Operations, Distribution, and Management Information and Support Systems. Participants can also count on extensive exposure to the company's international operations in Africa, Europe, Latin America and Asia as they proceed through the rotation system. 
Representative position descriptions for most of the initial assignments in the functional areas are attached.

\section{PREREQUISITES}

- Excellent communication skills, initiative, leadership potential, integrity, interpersonal skills, demonstrated self-management and professionalism, enthusiasm, and knowledge of various consumer products industries that sell through established retailers.

- Candidates should have superior proficiency in Excel as well as a basic understanding of retrieving and analyzing data retrieved from central data repositories using financial, accounting variance and statistical analysis tools to construct management reports.

- Candidates should also have capacity to build effective management presentations using PowerPoint or equivalent presentation software.

- Candidate for a Bachelor's Degree in Business Administration with a major in:

○ Accounting,

○ Economics,

Finance,

○ Management,

○ Marketing, or

○ MIS

- Ability to relocate upon completion of the program.

The MDP accepts a predetermined number of applicants each year.

\section{SAMPLE FUNCTIONAL POSITION DESCRIPTIONS}

\section{CORPORATE ACCOUNTING}

The company's Accounting department is directly responsible for monitoring the core general ledger module, including all subsidiaries and other off-site locations. MDP graduates move directly into Assistant Manager Positions responsible for ensuring that the financial data generated by the accounting system complies with Federal and State regulations, generally accepted Accounting principles, Governmental Accounting Standards Board (GASB) standards, and other corporate policy. Areas of responsibility include accounts receivable, accounts payable, fixed assets, payroll, foreign currency translation, and taxation. Assignments range from the corporate office to various subsidiaries, both in the U.S. and in the company's international operations. Several key accounting managers are past graduates of the Management Development Program. 


\section{FINANCIAL BUDGETING AND ANALYSIS}

The Financial Budgeting and Analysis department is responsible for aiding line departments in the preparation of budgets, preparing monthly and on-demand reporting of performance against budget, preparing and analyzing capital budgeting requests and managing cash and other investments. Financial personnel also play an important role in analyzing potential acquisition candidates. Graduates of the MDP move directly into an Assistant Manager position in one of these functions, typically with one of the regional or subsidiary operations. The acquisition team for our most recent acquisition in Spain was headed by a recent graduate of the Management Development Program.

\section{MARKETING}

The Marketing department is responsible for all of the company's marketing and promotion activities, including advertising on national, regional and local levels, on-site customer merchandising and promotion, product planning and development in coordination with the company's Research \& Development (R\&D) department, market analysis, market surveys, and day-to-day support of marketing and sales activities with the sales department. Since the company sells its products primarily through retail channels and very little directly to the end consumer, marketing works closely with our corporate customers to track end user demand and needs. Graduates of the Management Development Program assume positions as product managers, local and regional advertising or promotion managers, or assistant managers of departments responsible for regional or international market analysis and surveys.

\section{SALES}

The Sales Team works on the font line of a fiercely competitive global landscape, dealing with the most important people in our business - our customers. The team works with customers, both new and existing, to grow our highly profitable consumer products business armed with some of the most powerful trademarks in the world. Our sales approach focuses on fact-based selling and expert advice - offering "total product solutions," not just successful day-to-day transactions. MDP trainees work directly with the sales team, performing analysis, developing presentations, and managing customer accounts. Upon completion of the MDP, positions on the sales team are offered to those who exhibit superior selling skills. Generally, graduates assume team leadership responsibility for customer accounts in a particular sales area. Future assignments include the possibility of area, regional, or country manager positions. Several account managers are graduates of the Management Development Program. 


\section{HUMAN RESOURCES}

The Human Resources Department is responsible for coordinating the employment process, including the recruitment, application and interview process. In addition, other responsibilities assigned to HR include recruiting, benefits administration, workers' compensation (administration, claims processing, reporting), training and development, and management of the recurring semi-annual performance appraisal process for all company employees. For the latter, it is HR's responsibility to see that appraisals are conducted on a timely basis, appropriate records are maintained, and that appraisals are conducted in a fair and impartial manner. Generally graduates of the MDP assume assistant director positions in one of these responsibility areas, with advancement to HR director for one of the company's subsidiaries or regional offices within three years. Over half of the company's local HR directors are recent graduates of the Management Development Program.

\section{OPERATIONS}

The company's Operations group is responsible for all purchasing/procurement activities as well as for operations throughout the world. Operations' responsibilities include supplychain management and coordination, scheduling, service operations, quality assurance, labor relations, and the efficient and effective operations of manufacturing plants scattered around each region of the world in which the company does business. MDP graduates assume first-line supervisor positions in manufacturing or assembly plants or assistant manager positions in one of the supporting functions in operations. Several key operations staff managers are graduates of the MDP as are several plant managers.

\section{DISTRIBUTION}

The company maintains its own fleet of trucks to assure delivery and stocking of products in customer facilities throughout both North America and Europe. Regional distribution centers are also placed around the major countries in which the company does business. Distribution works closely with the sales team to assure on-time delivery of our product in an effort to maintain appropriate inventory quantities in customer locations, while minimizing shelf time to preserve product freshness and quality. Distribution also works closely with the Marketing Department to coordinate appropriate shelf displays with local and regional promotions. Positions offered to MDP graduates include management 
positions in purchasing/ procurement, supplier coordination, dispatching, scheduling, and inventory control. Several MDP graduates from earlier years hold middle management positions in the company's Distribution function.

\section{MANAGEMENT INFORMATION AND SUPPORT SYSTEMS}

Timely information has become the "life-blood" of most businesses and ours is no exception. As many of our customers, particularly those in the retail business, look to improve the performance of their supply chain operations, the need to integrate our information processing systems with the customer becomes paramount. Likewise, all of us look to increase the speed of response by minimizing the touch-points, departmental hand-offs, and volume of paper in day-to-day transactions. The company has invested heavily in state-of-the-art information processing technology to network and connect disparate processing systems within the firm and to build real-time linkages with our suppliers and customers. The MISS department is responsible for managing our data repositories, designing and maintaining our networked operating systems between suppliers and our internal departments, and between us and our customers, and for acquiring/developing software solutions to increase the efficiency and effectiveness of our operations. Upon completion of the MDP, graduates assume team leader positions in MISS, responsible for managing day-to-day operations of a current information and support system, or responsible for the acquisition/development of a new system to be put in place. A graduate from the $2004 \mathrm{MDP}$ assumed project management responsibility for the selection and implementation of the new CRM system brought on line this past spring. 


\section{References}

Adams, J. S. (1965). Inequity of social exchange. In L. Berkowitz (Ed.) Advances in experimental social psychology, (Vol. 2) (267-299). New York, NY: Academic Press.

Albrechtsen, J. S., Meissner, C. A., \& Susa, K. J. (2009). Can intuition improve deception detection performance? Journal of Experimental Social Psychology, 45(4), 1052-1055.

Allport, G. W. (1937). Personality: A psychological interpretation. New York, NY: Holt.

Ambady, N. (2010). The perils of pondering: Intuition and thin slice judgments. Psychological Inquiry, 21(4), 271-278.

Ambady, N., \& Gray, H. M. (2002). On being sad and mistaken: Mood effects on the accuracy of thin-slice judgments. Journal of Personality and Social Psychology, 83(4), 947-961.

Ambady, N., Hallahan, M., \& Conner, B. (1999). Accuracy of judgments of sexual orientation from thin slices of behavior. Journal of Personality and Social Psychology, 77(3), 538547.

Ambady, N., Krabbenhoft, M. A., \& Hogan, D. (2006). The 30-sec sale: Using thin-slice judgments to evaluate sales effectiveness. Journal of Consumer Psychology, 16(1), 4-13.

Ambady, N., \& Rosenthal, R. (1992). Thin slices of expressive behavior as predictors of interpersonal consequences: A meta-analysis. Personnel Psychology, 111(2), 256-274.

Ambady, N. \& Rosenthal, R. (1993). Half a minute: predicting teacher evaluations from thin slices of nonverbal behavior and physical attractiveness. Journal of Personality and Social Psychology, 64(3), 431-441. 
Ames, D. R., Kammrath, L. K., Suppes, A., \& Bolger, N. (2010). Not so fast: the (not-quitecomplete) dissociation between accuracy and confidence in thin-slice impressions. Personality and Social Psychology Bulletin, 36(2), 264-277.

Anderson, C. A., Lindsay, J. L., \& Bushman, B. J. (1999). Research in the psychological laboratory: Truth or triviality? Current Directions in Psychological Science, 8(1), 3-9.

Anderson, C. W. (1960). The relation between speaking times and decision in the employment interview. Journal of Applied Psychology, 44(4), 267-268.

Anderson, P. A. (1983). Decision making by objection and the Cuban Missile Crisis. Administrative Science Quarterly, 28(2), 201-222.

Arvey, R. D., \& Campion, J. E. (1982). The employment interview: A summary and review of recent research. Personnel Psychology, 35(2), 281-322.

Atkinson, R. C., \& Shiffrin, R. M. (1968). Human memory: A proposed system and its control processes. In K. W. Spence \& T. J. Spence (Eds.), The psychology of learning and motivation (Vol. 2). New York, NY: Academic Press.

Babad, E. (2005). Guessing teachers' differential treatment of high- and low-achievers from thin slices of their public lecturing behavior. Journal of Nonverbal Behavior, 29(2), 125-134

Baker, G., Gibbons, R., \& Murphy, K. J. (1994). Subjective performance measures in optimal incentive contracts. The Quarterly Journal of Economics, 109(4), 1125-1156.

Banker, R. D., Lee, S-Y., Potter, G., \& Srinivasan, D. (1996). Contextual analysis of performance impacts of outcome-based incentive compensation. Academy of Management Journal, 39(4), 920-948. 
Barling, J. \& Russell, B. (1983). Self-efficacy beliefs and sales performance. Journal of Organizational Behavior Management, 5(1), 41-51.

Barnes, C. M., Hollenbeck, J. R., Jundt, D. K., DeRue, D. S., \& Harmon, S. J. (2011). Mixing individual incentives and group incentives: Best of both worlds or social dilemma? Journal of Management. 37(6), 1611-1635.

Barrett, L. F., Tugade, M. M., \& Engle, R W. (2004). Individual differences in working memory capacity and dual-process theories of the mind. Psychological Bulletin, 130(4), 552-573.

Barrick, M. R., \& Mount, M. K. (1991). The big five personality dimensions and job performance: A meta-analysis. Personnel Psychology, 44(1), 1-26.

Barrick, M. R., Shaffer, J. A., \& DeGrassi, S. W. (2009). What you see may not be what you get: Relationships among self-presentation tactics and ratings of interview and job performance. Journal of Applied Psychology, 94(6), 1394-1411.

Barrick, M. R., Swider, B. W., \& Stewart, G. L. (2010). Initial evaluations in the interview: Relationships with subsequent interviewer evaluations and employment offers. Journal of Applied Psychology, 95(6), 1163-1172.

Baron, R. M., \& Kenny, D. A. (1986). The moderator-mediator variable distinction in social psychological research: Conceptual, strategic, and statistical considerations. Journal of Personality and Social Psychology, 51(6), 1173-1182

Bateman, M. J., \& Ludwig, T. D. (2004). Managing distribution quality through an adapted incentive program with tiered goals and feedback. Journal of Organizational Behavior Management, 23(1), 33-55. 
Bazerman, M. H. (1990). Judgment in managerial decision making. New York, NY: Wiley.

Bebchuk, L. A., \& Fried, J. M. (2004). Pay without performance: The unfulfilled promise of executive compensation. Cambridge, MA: Harvard University Press.

Becker, B., \& Gerhart, B. (1996). The impact of human resource management on organizational performance: Progress and prospects. Academy of Management Journal, 39(4), 779-801.

Berkson, H. M., Ferris, G. R., \& Harris, M. M. (2002). The recruitment interview process: Persuasion and organization reputation promotion in competitive labor markets. Human Resource Management Review, 12(3), 359-375.

Bernieri, F., Gillis, J. S., Davis, J. M., \& Grahe, J. E. (1996). Dyad rapport and the accuracy of its judgment across situations: A lens model analysis. Journal of Personality and Social Psychology, 71(1), 110-129.

Berry, K. J., \& Mielke, P. W. Jr. (2002). Least sum of Euclidean regression residuals: Estimation of effect size. Psychological Reports, 91(3), 955-962.

Binet, A. (1911). Nouvelles researchers sur la mesure du niveau intellectual chez les enfants d'école. L'Annu Psychologique, 17(1), 145-201.

Blanck, P. D., Rosenthal, R., Viannicelli, M., \& Lee, T. D. (1986). Therapists' tone of voice: Descriptive, psychometric, interactional, and competence analyses. Journal of Social and Clinical Psychology, 4(2), 154-178.

Borkenau, P., Mauer, N., Riemann, R., Spinath, F. M., \& Angleitner, A. (2004). Thin slices of behavior as cues of personality and intelligence. Journal of Personality and Social Psychology, 86(4), 599-614. 
Bourne, L. E., Dominowski, R. L., \& Loftus, E. F. (1979). Cognitive Processes. Englewood Cliffs, NJ: Prentice-Hall.

Brainerd, C. J. \& Kingma, J. (1984). Do children have to remember to reason? A fuzzy trace theory of transitive development. Developmental Review, 4(4), 311-377.

Brainerd, C. J., \& Reyna, V. F. (1990). Gist is the grist: Fuzzy-trace theory and the new intuitionism. Developmental Review. 10(1), 3-47.

Brainerd, C. J., \& Reyna, V. F. (2001). Fuzzy trace theory: Dual processes in memory, reasoning and cognitive neuroscience. Advances in Child Development, 28(1), 41-100.

Bransford, J. D, \& Franks, J. J. (1971). The abstraction of linguistic ideas. Cognitive Psychology, 2(4), 193-209.

Breaugh, J., \& Starke, M. (2000). Research on employee recruitment: So many studies, so may remaining question. Journal of Management, 26(3), 405-434.

Breaux, D. M., Munyon, T. P., Hochwarter, W. A., \& Ferris, G. R. (2009). Politics as a moderator of the accountability-job satisfaction relationship: Evidence across three studies. Journal of Management, 35(2), 307-326.

Brouer, R. L., Harris, K. J., \& Kacmar, K. M. (2011). The moderating effects of political skill on the perceived politics-outcome relationship. Journal of Organizational Behavior, 32(6), 869-885.

Brouwer, L. E. J. (1952). Historical background, principles and methods of intuitionism. South African Journal of Science, 49(2), 139-146. 
Brown, R. (2004). Consideration of the origin of Herbert Simon's Theory of "Satisficing” (19331947). Management Decision, 42(10), 1240-1256.

Bryant, F. B., \& Sattora, A. (2013) EXCEL macro file for conducting scaled difference chisquare tests via LISREL 8, LISREL 9, EQS, and Mplus. Available from the authors.

Butler, S. A. (1986). Anchoring in the judgmental evaluation of audit samples. Accounting Review, 61(1), 101-111.

Byrne, D. (1971). The attraction paradigm. New York, NY: Academic Press.

Cable, D. M., \& Judge, T. A. (1997). Interviewers' perceptions of person-organization fit and organizational selection decisions. Journal of Applied Psychology, 82(4), 546-561.

Cable, D. M., \& Yu, K. Y. T. (2006). Managing job seekers' organizational image beliefs: The role of media richness and media credibility. Journal of Applied Psychology, 91(4), 828840.

Campion, M. A., Palmer, D. K., \& Campion, J. E. (1997). A review of structure in the selection interview. Personnel Psychology, 50(3), 655-702.

Carney, D. R., Colvin, C. R., \& Hall, J. A. (2007). A thin slice perspective on the accuracy of first impressions. Journal of Research in Personality, 41(5), 1054-1072.

Carrère, S., \& Gottman, J. M. (1999). Predicting divorce among newlyweds from the first three minutes of a marital conflict discussion. Family Process, 38(3), 293-301. 
Cervone, D., \& Peake, P. K., (1986). Anchoring, efficacy, and action: The influence of judgmental heuristics on self-efficacy judgments and behavior. Journal of Personality and Social Psychology, 50(3), 492-501.

Chaiken, S. (1980). Heuristic versus systematic information processing and the use of source versus message cues in persuasion. Journal of Personality and Social Psychology, 39(5), $752-766$.

Chapman, D. S., \& Zweig, D. I. (2005). Developing a nomological network for interview structure: Antecedents and consequences of the structured selection interview. Personnel Psychology, 58(3), 673-702.

Choi, J., \& Chen, C. C. (2007). The relationships of distributive justice and compensation system fairness to employee attitudes in international joint ventures. Journal of Organizational Behavior, 28(6), 687-703.

Coase, R. (1998). The new institutional economics. AER Papers and Proceedings, 88, 72-74.

Cohen, J. (1994). The earth is round ( $p>.05)$. American Psychologist, 49(12), 997-1003.

Commons, J. R. (1934). Institutional economics - Its place in political economy. New York, NY: Macmillan.

Colquitt, J. A. (2014). Lab vs. field OB: Do findings converge? Presentation for the Center for the Advancement of Research Methods and Analysis.

Colquitt, J. A., Conlon, D. E., Wesson, M. J., Porter, C. O., \& Ng, K. Y. (2001). Justice at the millennium: A meta-analytic review of 25 years of organizational justice research. Journal of Applied Psychology, 86(3), 425-445. 
Colquitt, J. A., LePine, J. A., \& Noe, R. A. (2000). Toward an integrative theory of training motivation: A meta-analytic path analysis of 20 years of research. Journal of Applied Psychology, 85(5), 678-707.

Condly, S. J., Clark, R. E., \& Stolovitch, H. D. (2003). The effects of incentives on workplace performance: A meta-analytic review of research studies. Performance Improvement Quarterly, 16(3), 46-63.

Connerly, M. L. (1997). The influence of training on perceptions of recruiters' interpersonal skills and effectiveness. Journal of Occupational and Organizational Psychology, 70(3), 259-272.

Conway, J. M., Jako, R. A., \& Goodman, D. F. (1995). A meta-analysis of interrater and internal consistency reliability of selection interviews. Journal of Applied Psychology, 80(5), 565579.

Conway, J. M., \& Peneno, G. M. (1999). Comparing structured interview question types: Construct validity and applicant reactions. Journal of Business and Psychology, 13(4), 485-506.

Cortina, J. M. (2003). Apples and oranges (and pears, oh my!): The search for moderators in meta-analysis. Organizational Research Methods, 6(4), 415-439.

Cowan, N. (1988). Evolving conceptions of memory storage, selective attention, and their mutual constraints within the human information-processing system. Psychological Bulletin, 104(2), 163-191. 
Craik, F. I. M., \& Lockhart, R. S. (1972). Levels of processing: A framework for memory research. Journal of Verbal Learning and Verbal Behavior, 11(6), 671-684.

Curhan, J. R., \& Pentland, A. (2007). Thin slices of negotiation: Predicting outcomes from conversational dynamics within the first 5 minutes. Journal of Applied Psychology, 92(3), 802-811.

Dalessio, A. T., \& Imada, A. S. (1984). Relationships between interview selection decisions and perceptions of applicant similarity to an ideal employee and self: A field study. Human Relations, 37(1), 67-80.

Dalessio, A. T., \& Silverhart, T. A. (1994). Combining biodata test and interview information: Predicting decision and performance criteria. Personnel Psychology, 47(2), 303-315.

Daniel, C., \& Valencia, S. (1991). Structured interviewing simplified. Public Personnel Management, 20(2), 197-134.

Dean, A. L., Chabaud, S., \& Bridges, E. (1981). Classes, collections, and distinctive features: Alternative strategies for solving inclusion problems. Cognitive Psychology, 13(1), 84112.

Deci, E. L., Koestner, R., \& Ryan, R. M. (1999). A meta-analytic review of experiments examining the effects of extrinsic rewards on intrinsic motivation. Psychological Bulletin, $125(6), 627-668$.

Deci, E. L., \& Ryan, R. M. (1985). Intrinsic motivation and self-determination in human behavior. New York, NY: Plenum

Deci, E. L., \& Ryan, R. M. (2010). Self-determination. Corsini Encyclopedia of Psychology, 1-2. 
Dijksterhuis, A., Bos, M. W., Nordgren, L. F., \& von Baaren, R. B. (2006). On making the right choice: The deliberation-without-attention effect. Science, 311, 1005-1007.

Dipboye, R. L. (1994). Structured and unstructured selection interviews: Beyond the job-fit model. In Ferris, G. R. (Ed.) Research in personnel and human resources management (Vol. 12) (79-123). Greenwich, CT: JAI Press.

Dipboye, R. L., \& Flanagan, M. F. (1979). Research settings in industrial and organizational psychology: Are findings in the field more generalizable than in the laboratory? American Psychologist, 34(2), 141-150.

Doeringer, P. B., \& Piore, M. J. (1971). Internal labor markets and manpower analysis. Lexington, MA: D.C. Heath.

Dougherty, T. W., \& Turban, D. B. (1999). Behavioral confirmation of interviewer expectancies. In R.W. Eder, \& M.M. Harris (Eds.), The employment interview handbook (217-228). Thousand Oaks, CA: Sage.

Eisenberger, R., \& Cameron, J. (1996). Detrimental effects of rewards: Reality or myth? American Psychologist, 51(11), 1153-1166.

Eisenhardt, K. (1989). Making fast strategic decisions in high-velocity environments. Academy of Management Journal, 32(3), 543-576.

Epstein, S (1994). Integration of the cognitive and psychodynamic unconscious. American Psychologist, 49(8), 709-724. 
van Essen, M., Heugens, P., Otten, J., \& van Oosterhout, J. (2012). An institution-based view of executive compensation: A multilevel meta-analytic test. Journal of International Business Studies, 43(4), 396-423.

Estes, W. K. (1988). Toward a framework for combining connectionist and symbol-processing models. Journal of Memory and Language, 27(2), 196-212.

Evans, J. St. B. T. (1989). Bias in human reasoning: Causes and consequences. Brighton, UK: Erlbaum.

Evans, J. St. B. T. (2003). In two minds: Dual process accounts of reasoning. Trends in Cognitive Sciences, 7(10), 454-459.

Evans, J. St. B. T. (2008). Dual processing accounts of reasoning, judgment, and social cognition. Annual Review of Psychology, 59, 255-278.

Evans, J. St. B. T. (2010). Thinking twice: Two minds in one brain. Oxford, UK: Oxford University Press.

Evans, J. St. B. T., Barston, J. L., \& Pollard, P. (1983). On the conflict between logic and belief in syllogistic reasoning. Memory \& Cognition, 11(3), 295-306.

Fiske, S. T., \& Taylor, S. E. (1984). Social cognition. New York, NY: Random House.

Floyd, S. W., \& Wooldridge, B. (1997). Middle management's strategic influence and organizational performance. Journal of Management Studies, 34(3), 465-485.

Fodor, J. (1983). Modularity of mind: An essay on faculty psychology. Cambridge, MA: The MIT Press. 
Folger, R., \& Konovsky, M. A. (1989). Effects of procedural and distributive justice on reactions to pay raise decisions. Academy of Management Journal, 32(1), 115-130.

Forbes, D. P., \& Milliken, (1999). Cognition and corporate governance: Understanding boards of directors as strategic decision making groups. Academy of Management Review, 24(3), 489-505.

Ford, K. J., Schmitt, N., Schechtman, S. L., Hults, B. M., \& Doherty, M. L. (1989). Process tracing methods: Contributions, problems, and neglected research questions. Organizational Behavior and Human Decision Processes, 43(1), 75-117.

Fowler, K. A., Lilienfield, S. O., \& Patrick, C. J. (2009). Detecting psychopathy from thin slices of behavior. Psychological Assessment, 21(1), 68-78.

Frederick, S. (2005). Cognitive reflection and decision making. Journal of Economic Perspectives, 19(4), 25-42.

Frege, G. (1884). Die Grundlagen der arithmetik. Breslau: M. \& H. Marcus.

Friedman, J. N. W., Oltmanns, T. F., \& Turkheimer, E. (2007). Interpersonal perception and personality disorders: Utilization of a thin slice approach. Journal of Research in Personality, 41(3), 667-688.

Funder, D. C. (2001). Accuracy in personality judgment: Research and theory concerning an obvious question. In B. W. Robertss \& R. Hogan (Eds.), Personality in the Workplace. Decade of Behavior (pp. 120-140). Washington, D.C.: American Psychological Association. 
Garb, H. N. (1989). Clinical judgment, clinical training, and professional experience. Psychological Bulletin, 105(3), 387-396.

Garcia, M. F., Posthuma, R. A., \& Colella, A. (2008). Fit perceptions in the employment interview: The role of similarity, liking, and expectations. Journal of Organizational and Occupational Psychology, 81(2), 173-189.

Gatewood, R. D., \& Field, H. S. (1987). Human Resource Selection. New York, NY: The Dryden Press.

Gatewood, R., Lahif, J., Deter, R., \& Hargrove, L. (1989). Effects of training on behaviors of the selection interview. Journal of Business Communication, 26(1), 17-31.

Garbers, Y., \& Konradt, U. (2014). The effect of financial incentives on performance: A quantitative review of individual and team-based financial incentives. Journal of Occupational and Organizational Psychology, 87(1), 102-137.

Gerhart, B., \& Milkovich, G. T. (1992). Employee compensation: Research and theory. In M. D. Dunnette \& L. M. Hough (Eds.). Handbook of industrial and organizational psychology ( $2^{\text {nd }}$ ed.) (Vol. 3) (pp. 481-569). Paolo Alto, CA: Consulting Psychologists Press.

Gerhart, B., \& Rynes, S. L. (2004). Compensation: Theory, evidence and strategic implications. Thousand Oaks, CA: Sage.

Gerhart, B., Trevor, C., \& Graham, M. (1996). New directions in employee compensation research. In G. R. Ferris (Ed.), Research in personnel and human resource management (Vol. 14) (pp. 143-203). Greenwich, CT: JAI Press. 
Ghiselli, E. E., Campbell, J. P., \& Zedeck, S. (1981). Measurement theory for the behavioral sciences. New York, NY: W. H. Freeman.

Goldberg, L. R. (1992). The development of markers for the Big-Five factor structure. Psychological Assessment, 4(1), 26-42.

Gomez-Mejia, L., \& Wiseman, R. M. (1997). Reframing executive compensation: An assessment and outlook. Journal of Management, 23(3), 291-374.

Goodman, P. S. (1974). An examination of referents used in the evaluation of pay. Organizational Behavior and Human Performance, 12(2), 170-195.

Gottman, J. M. (1979). Marital interaction: Experimental investigations. New York, NY: Academic Press.

Graves, L. M., \& Powell, G. N. (1995). The effects of sex similarity on recruiters' evaluations of actual applicants: A test of the similarity-attraction paradigm. Personnel Psychology, 48(1), 85-98.

Griffitts, C. H. (1924). Supplementing the interview by tests. In Fundamentals of Vocational Psychology (Vol. 4) (pp. 147-164), New York, NY: MacMillan Co.

Hackman, J. R., \& Oldham, G. R. (1976). Motivation through design of work: Test of a theory. Organizational Behavior and Human Performance, 16(2), 250-279.

Hall, A., T., Frink, D. D., Ferris, G. R., Hochwarter, W. A., Kacmar, C. J., \& Bowen, M. G. (2003). Accountability in human resources management. In C. A. Schriesheim \& L. Neider (Eds.), New directions in human resource management: (pp. 29-63). Greenwich, CT: Information Age Publishing. 
Hammond, K. R. (1996). Human judgment and social policy: Irreductible uncertainty, inevitable error, unavoidable justice. New York, NY: Oxford University Press.

Harlow, H. F. (1949). The formation of learning sets. Psychological Review, 56(1), 51-65.

Harris, M. M. (1989). Reconsidering the employment interview: A review of recent literature and suggestions for future research. Personnel Psychology, 42(4), 691-726.

Herold, K. P. P. (1995). The effects of an interviewee's self disclosure and disability on selected perceptions and attitudes of interviewers. Unpublished doctoral dissertation, University of Southern Mississippi, Hattiesburg.

Herzberg, F. (1959). The motivation to work. New York, NY: John Wiley and Sons.

Heslin, P. A. \& VandeWalle, D. (2011). Performance appraisal procedural justice: The role of a manager's implicit person theory. Journal of Management, 37(6), 1694-1718.

Heyting, H. (1959). Constructivity in Mathematics. Amsterdam: North-Holland

Highhouse, S. (2008). Stubborn reliance on intuition and subjectivity in employee selection. Industrial and Organizational Psychology, 1(3), 333-342.

Hilbert, D. (1923). Die logischen grundlagen der mathematic. Mathematische Annalen, 88(1), $151-165$.

Hitt, M. A., Keats, B., \& DeMarie, S. M. (1998). Navigating in the new competitive landscape: Building strategic flexibility and competitive advantage in the $21^{\text {st }}$ century. Academy of Management Executive, 12(4), 22-42. 
Hollenbeck, J. R., \& Klein, H. J. (1987). Goal commitment and the goal-setting process:

Problems, prospects, and proposals for future research. Journal of Applied Psychology, $72(2), 212-230$.

Holleran, S. E., Mehl, M. R., \& Levitt, S. (2009). Eavesdropping on social life: The accuracy of stranger ratings of daily behavior from thin slices of natural conversations. Journal of Research in Personality, 43(3), 660-672.

Hu, L., \& Bentler, P. M. (1999). Cutoff criteria for fit indexes in covariance structure analysis: Conventional criteria versus new alternatives. Structural Equation Modeling, 6(1), 1-55.

Huber, P. J. (1967). The behavior of maximum likelihood estimates under nonstandard conditions. In Proceedings of the fifth Berkeley symposium on mathematical statistics and probability. (Vol. 1) (pp. 221-233) Berkeley, CA: University of California Press.

Huffcutt, A. I. (2011). An empirical review of the employment interview construct literature. International Journal of Selection and Assessment, 19(1), 62-81.

Huffcutt, A. I., \& Arthur, W., Jr. (1994). Hunder and Hunter (1984) Revisited: Interview validity for entry-level jobs. Journal of Applied Psychology, 79(2), 184-190.

Huffcutt, A. I., \& Culbertson, S. S. (2010). Interviews. In Zedek S (Ed.), APA handbook of industrial and organizational psychology (pp. 185-203). Washington, D.C.: American Psychological Association.

Huffcutt, A. I., Culbertson, S. S., \& Weyhrauch, W. S. (2013). Employment interview reliability: New meta-analytic estimates by structure and format. International Journal of Selection and Assessment, 21(3), 264-276. 
Huffcutt, A. I., \& Roth, P. L. (1998). Racial group differences in employment interview evaluations. Journal of Applied Psychology, 83(2), 179-189.

Huffcutt, A. I., Weekley, J. A., Wiesner, W. H., DeGroot, T. G., \& Jones, C. (2001). Comparison of situational and behavior description interview questions for higher-level positions. Personnel Psychology, 54(3), 619-644.

Hunter, J. E., \& Hunter, R. F. (1984). Validity and utility of alternative predictors of job performance. Psychological Bulletin, 53(1), 119-131.

Ilgen, D. R. (1989). Laboratory research: A question of when, not if. In E. A. Locke's (Ed.), Generalizing from laboratory to field setting (pp. 269-279) .Lexington, MA: Lexington Books.

Ivancevich, J. M., \& McMahon, J. T. (1982). The effects of goal setting, external feedback, and self-generated feedback on outcome variables: A field experiment. Academy of Management Journal, 25(5), 359-372.

Jacobs, R., Kafry, D., \& Zedeck, S. (1980). Expectations of behaviorally anchored rating scales. Personnel Psychology, 33(3), 595-640.

Janz, T. (1982). Initial comparisons of patterned behavior description interviews versus unstructured interviews. Journal of Applied Psychology, 67(5), 577-580.

Janz, T. (1989). The patterned behavior description interview: The best prophet of the future is the past. In Eder, R. W., \& Ferris, G. R. (Eds.), The employment interview: Theory, research, and practice, (pp. 158-168). Newbury Park, CA: Sage. 
Jenkins, D. G. Jr., Mitra, A., Gupta, N., \& Shaw, J. D. (1998). Are financial incentives related to performance? A meta-analytic review of empirical research. Journal of Applied Psychology, 83(5), 777-787.

Johnson, M. K., \& Sherman, S. J. (1990). Constructing and reconstructing the past and future in the present. In E. T. Higgins \& R. M. Sorrentino (Eds.), Handbook of motivation and cognition: Foundations of social behavior (Vol 2) (pp. 82-526). New York, NY: Guilford Press.

Jonsson, A., \& Allwood, C. M. (2003). Stability and variability in the realism of confidence judgments over time content domain, and gender. Personality and Individual Difference, $34(4), 559-574$.

Judge, T. A., Bono, J. E. (2001). Relationship of core self-evaluation traits - self-esteem, generalized self-efficacy, locus of control, and emotional stability - with job satisfaction and job performance: A meta-analysis. Journal of Applied Psychology, 86(1), 80-92.

Judge, T. A., Heller, D., \& Mount, M. K. (2002). Five-factor model of personality and job satisfaction: A meta-analysis. Journal of Applied Psychology, 87(3), 530-541.

Kahneman, D. \& Tversky, A. (1979). Prospect theory: An analysis of decision under risk. Econometrica, 47(2), 263-291.

Karau, S. J., \& Williams, K. D. (1993). Social loafing: A meta-analytic review and theoretical integration. Journal of Personality and Social Psychology. 65(4), 681-706. 
Kataoka, H. C., Latham, G. P., \& Whyte, G. (1997). The relative resistance of the situational, patterned behavior, and conventional structured interviews to anchoring effects. Human Performance, 10(1), 47-63.

Kaul, T. J., \& Schmidt, L. D. (1971). Dimensions of interviewer trustworthiness. Journal of Counseling Psychology, 18(6), 542-548.

Keeney, R. \& Raiffa, H. (1993). Decisions with multiple objectives: Preferences and value tradeoffs. New York, NY: Cambridge University Press.

Kenny, D. A. (1979). Correlation and causation. New York, NY: Wiley.

Kerr, C. (1954). The balkanization of labor markets. In E. W. Bakke (Ed.), Labor mobility and economic opportunity (pp. 92-110). New York, NY: John Wiley

Kerr, S. (1975). On the folly of rewarding for A, while hoping for B. Academy of Management Journal, 18(4), 769-783.

Kidwell, R. E. Jr., \& Bennett, N. (1993). Employee propensity to withhold effort: A conceptual model to intersect three avenues of research. Academy of Management Review. 18(3), 429-456.

Kingstrom, P. O., \& Bass, A. R. (1981). A critical analysis of studies comparing behaviorally anchored rating scales (BARS) and other rating formats. Personnel Psychology, 34(2), 263-289.

Kinicki, A. J., Lockwood, C. A., Hom, P. W., \& Griffeth, R. W. (1990). Interviewer predictions of applicant qualifications and interviewer validity: Aggregate and individual analyses. Journal of Applied Psychology, 75(5), 477-486. 
Kintsch, W. (1988). The role of knowledge in discourse comprehension: A constructionintegration model. Psychological Review, 95(2), 163-182.

Klauer, K. C, Musch, J., \& Naumer, B. (2000). On belief bias in syllogistic reasoning. Psychological Review, 107(4), 852-884.

Klehe, U., \& Latham, G. (2005). The predictive and incremental validity of the situational and patterned behavior description interviews for teamplaying behavior. International Journal of Selection and Assessment, 13(2), 108-115.

Klein, H. J., Wesson, M. J., Hollenbeck, J. R., \& Alge, B. J. (1999). Goal commitment and the goal-setting process: Conceptual clarification and empirical synthesis. Journal of Applied Psychology, 84(6), 885-896.

Klimoski, R. J., \& London, M. (1974). Role of the rater in performance appraisal. Journal of Applied Psychology, 59(4), 157-162.

Kolodner, J. L. (1983). Reconstructive memory: A computer model. Cognitive Science, 7(4), 281-328.

Krajewski, H. T., Goffin, R. D., McCarthy, J. M., Rothstein, M. G., \& Johnson, N. (2006). Comparing the validity of structured interviews for managerial-level employees: Should we look to the past or focus on the future. Journal of Occupational and Organizational Psychology, 79(3), 411-432.

Kraus, M. W., \& Keltner, D. (2009). Signs of socioeconomic status: A thin-slicing approach. Psychological Science, 20(1), 99-106. 
Krefting, L. A., \& Brief, A. P. (1976). The impact of applicant disability on evaluative judgments in the selection process. Academy of Management Journal, 40(4), 675-680.

Kristof, A. L., (1996). Person-organization fit: An integrative review of its conceptualization, measurement, and implications. Personnel Psychology, 49(1), 1-49.

Kristof-Brown, A. L., Zimmerman, R. D., \& Johnson, E. C. (2005). Consequences of individuals' fit at work: A meta-analysis of person-job, person-organization, persongroup, and person-supervisor fit. Personnel Psychology, 58(2), 281-342.

Kruse, D. L. (1993). Profit sharing: Does it make a difference? Kalamazoo, MI: Upjohn Institute.

Lakshmanan, A. \& Krishnan, H. S. (2009). How does imagery in interactive consumption lead to false memory? A reconstructive memory perspective. Journal of Consumer Psychology, $19(3), 451-462$.

Lambert, R. A., Larcker, D. F., \& Weigelt, K. (1993). The structure of organizational incentives. Administrative Science Quarterly, 38(3), 438-461.

Larsen, D. A., \& Phillips, J. I. (2002). Effect of recruiter on attraction to the firm: Implications for the elaboration likelihood model. Journal of Business and Psychology, 16(3), 347364.

Latham, G. P. (1989). The reliability, validity, and practicality of the situational interview. In Eder, R. W. \& Ferris, G. R. (Eds.), The employment interview: Theory, research, and practice (pp. 169-182). Newbury Park, CA: Sage. 
Latham, G. P., \& Finnegan, B. J. (1993). In Schuler, H., Farr, J. L., \& Smith, M. (Eds.) Personnel selection and assessment: Individual and organizational perspectives (pp. 4155). Hillsdale, NJ: Lawrence Erlbaum Associates.

Latham, G. P., Saari, L. M., Pursell, E. D., Campion, M. A. (1980). The situational interview. Journal of Applied Psychology, 65(4), 422-427.

Latham, G. P., Wexley, K. N., \& Pursell, E. D. (1975). Training managers to minimize rating errors in the observation of behavior. Journal of Applied Psychology, 60(5), 550-555.

LaTour, K. A., LaTour, M. S., \& Brainerd, C. (2014). Fuzzy trace theory and "smart" false memories: Implications for advertising. Journal of Advertising, 43(1), 3-17.

Lepper, M., Greene, D., \& Nisbett, R. (1973). Undermining children's intrinsic interest with extrinsic reward. Journal of Personality and Social Psychology, 28(1), 129-137.

Levashina, J., Hartwell, C. J., Morgeson, F. P., \& Campion, M. A. (2014). The structured employment interview: Narrative and quantitative review of the research literature. Personnel Psychology, 67(1), 241-293.

Lichtenstein, S., Fischoff, B., \& Phillips, L. D. (1982). Calibration of probabilities: The state of the art to 1980. In D. Kahneman, P. Slovic, \& A. Tversky (Eds.), Judgments under uncertainty: Heuristics and biases (pp. 306-334). New York, NY: Cambridge University Press.

Lieberman, M. D. (2000). Intuition: A social cognitive neuroscience approach. Psychological Bulletin, 126(1), 109-137. 
Lievens, F., De Corte, W., \& Brysee, K. (2003). Applicant perceptions of selection procedures: The role of selection information, belief in tests, and comparative anxiety. International Journal of Selection and Assessment, 11(1), 67-77.

Lievens, F., Highhouse, S., \& De Corte, W. (2005). The importance of traits and abilities in supervisors' hirability decisions as a function of method of assessment. Journal of Occupational and Organizational Psychology, 78(3), 453-470.

Lin, T. R., Dobbins, G. H., \& Farth, J. L. (1992). A field study of race and age similarity effects on interview ratings in conventional and situational interviews. Journal of Applied Psychology, 77(3), 363-371.

Lindenberger, U. \& Pötter, U. (1998). The complex nature of unique and shared effects in hierarchical linear regression: Implications for developmental psychology. Psychological Methods, 3(2), 218-230.

Lipp, E., Stanley, A., \& Lariviere, T. (2007). 2007 Recruiting Metrics and Performance Benchmark Report. Willow Grove, PA: Staffing.org.

Locke, E. (1968). Toward a theory of task motivation and incentives. Organizational Behavior and Human Performance, 3(2), 157-189.

Locke, E. A., \& Bryan, J. F. (1969). Knowledge of score and group level as determinants of work rate. Journal of Applied Psychology, 53(1), 59-65.

Locke, E. A., \& Latham, G. P. (1984). Goal setting: A motivational technique that works! Englewood Cliffs, NJ: Prentice Hall. 
Locke, E. A., \& Latham, G. P. (2002). Building a practically useful theory of goal-setting and task motivation. American Psychologist, 57(9), 705-717.

Logan, G. D. (1992). Shapes of reaction time distributions and shapes of learning curves: A test of the instance theory of automaticity. Journal of Experimental Psychology: Learning, Memory, and Cognition, 18(5), 883-914.

Lord, R. G., \& Maher, K. J. (1990). Alternative information-processing models and their implications for theory, research, and practice. The Academy of Management Review, $15(1), 9-28$.

Macan, T. H. (2009). The employment interview: A review of current studies and directions for future research. Human Resource Management Review, 19(3), 203-218.

Macan, T. H., \& Dipboye, R. L. (1990). The relationship of interviewers' preinterview impressions to selection and recruitment outcomes. Personnel Psychology, 43(4), 745768.

Macan, T. H., \& Dipboye, R. L. (1994). The effects of the application on processing of information from the employment interview. Journal of Applied Social Psychology, 24(14), 1291-1314.

MacKinnon, D. P. (2008). Introduction to statistical mediation analysis. Mahwah, NJ: Erlbaum.

MacKinnon, D. P., Lockwood, C. M., \& Williams, J. (2004). Confidence limits for the indirect effect: Distribution of the product and resampling methods. Multivariate Behavioral Research, 39(1), 99-128. 
Marchese, M. C., \& Muchinsky, P. M. (1993). The validity of the employment interview: A meta-analysis. International Journal of Selection and Assessment, 1(1), 18-26.

Marsh, H. W., Hau, K., \& Wen, Z. (2004). In search of golden rules: Comment on hypothesistesting approaches to setting cutoff values for fit indexes and dangers in overgeneralizing Hu and Bentler's (1999) findings. Structural Equation Modeling, 11(3), 320-341.

Martin, G. P., Gomez-Mejia, L. R., \& Wiseman, R. M. (2013). Executive stock options as mixed gambles: revisiting the behavioral agency model. Academy of Management Journal, $56(2), 451-472$.

Maslow, A. H. (1943). A theory of human motivation. Psychological Review, 50(4), 370-396.

Maurer, S. D. (2002). A practitioner-based analysis of interviewer job expertise and scale format as contextual factors in situational interviews. Personnel Psychology, 55(2), 30-327.

McDaniel, M. A., Schmidt, F. L., \& Hunter, J. E. (1988). A meta-analysis of the validity of methods for rating training and experience in personnel selection. Personnel Psychology, 41(2), 283-314.

McDaniel, M. A., Whetzel, D. L., Schmidt, F. L., \& Maurer, S. (1994). The validity of employment interviews: A comprehensive review and meta-analysis. Journal of Applied Psychology, 79(4), 599-616.

McFarlin, D. B., \& Sweeney, P. D. (1992). Distributive and procedural justice as predictors of satisfaction with personal and organizational outcomes. Academy of Management Journal, 35(3) 626-637. 
McNamara, G., Moon, H., \& Bromiley, P. (2002). Banking on commitment: Intended and unintended consequences of an organization's attempt to attenuate escalation of commitment. Academy of Management Journal, 45(2), 443-452.

Messersmith, J. G., Guthrie, J. P., Ji, Y-Y., \& Lee, J-Y. (2011). Executive turnover: The influence of dispersion and other pay system characteristics. Journal of Applied Psychology, 96(3), 457-469.

Meurs, J. A., \& Perrewé, P. L. (2011). Cognitive activation theory of stress: An integrative theoretical approach to stress. Journal of Management, 37(4), 1043-1068.

Milgrom, P., \& Roberts, J. (1992). Economics, organization \& management. Englewood Cliffs, NJ: Prentice Hall.

Miller, G. A. (1956). The magical number seven, plus or minus two: Some limits on our capacity for processing information. Psychological Review, 63(2), 81-97.

Miner, J. B. (1980). Theories of organizational behavior. Hinsdale, Il: Dryden.

Mintz, J., \& Luborsky, L. (1971). Segments versus whole sessions: Which is the better unit for psychotherapy process research? Journal of Abnormal Psychology, 78(2), 291-295.

Morgeson, F. P., Reider, M. H., Campion, M. A., \& Bull, R. A. (2008). Review of research on age discrimination in the employment interview. Journal of Business and Psychology, 22(3), 223-232.

Moscoso, S. (2000). Selection interview: A review of validity evidence, adverse impact and applicant reactions. International Journal of Selection and Assessment, 8(4), 237-247. 
Mowday, R. T. (1996). Equity theory predictions of behavior in organizations. In R. M. Steers, L. W. Porter, \& G. A. Bigley (Eds.), Motivation and leadership at work (pp. 53-71). New York, NY: McGraw-Hill.

Mullen, B., \& Copper, C. (1994). The relationship between group cohesiveness and performance: An integration. Psychological Bulletin. 115(2), 210-227.

Murphy, N. A. (2005). Using thin slices for behavioral coding. Journal of Nonverbal Behavior, 29(4), 235-246.

Murphy, K. R., Gannett, B. A., Herr, B. M., \& Chen, J. A. (1986). Effects of subsequent performance on evaluations of previous performance. Journal of Applied Psychology, $71(3), 427-431$.

Muthén, B. O., \& Muthén, L. K. (2012). Mplus User’s Guide. Los Angeles, CA: Muthén \& Muthén.

Netemeyer, R. G., Johnston, M. W., \& Burton, S. (1990). Analysis of role conflict and role ambiguity in a structural equations framework. Journal of Applied Psychology, 75(2), $148-157$.

Nisbett, R., Peng, K., Choi, I., \& Norenzayan, A. (2001). Culture and systems of thought: Holistic vs. analytic cognition. Psychological Review, 108(2), 291-310.

Northcraft, G. B., \& Neale, M. A. (1987). Amateurs, experts, and real estate: An anchoring-andadjustment perspective on property pricing decisions. Organizational Behavior and Human Decision Processes, 39(1), 84-97. 
Nyberg, A., Fulmer, I., Gerhart, B., \& Carpenter, M. (2010). Agency theory revisited: CEO return and shareholder interest alignment. Academy of Management Journal, 53(5), 10291049.

Oltmanns, T. F., Friedman, J. N. W., Fiedler, E. R., \& Turkheimer, E. (2004). Perceptions of people with personality disorders based on thin slices of behavior. Journal of Research in Personality, 38(3), 216-229.

Opsahl. R. L., \& Dunnette, M. D. (1966). The role of financial incentives in industrial motivation. Psychological Bulletin, 66(2), 95-116.

O’Reilly, C. A. (1980). Individuals and information overload in organizations: Is more necessarily better? Academy of Management Journal, 23(4), 684-696.

Paas, F., Renkl, A., \& Sweller, J. (2004). Cognitive load theory: Instructional implications of the interaction between information structures and cognitive architecture. Instructional Science, 32(1), 1-8.

Pearce, J. L., Stevenson, W. B., \& Perry, J. L. (1985). Managerial compensation based on organizational performance: A time series analysis of the effects of merit pay. Academy of Management Journal, 28(2), 261-278.

Pepper, A. \& Gore, J. (in press). Behavioral agency theory new foundations for theorizing about executive compensation. Journal of Management.

Perlow, L. A., Okhuysen, G. A., \& Repenning, N. P. (2002). The speed trap: Exploring the relationship between decision making and temporal context. Academy of Management Journal, 45(4), 931-955. 
Pfeffer, J., \& Langton, N. (1993). The effect of dispersion on satisfaction, productivity, and working collaboratively: Evidence from college and university faculty. Administrative Science Quarterly, 38(3), 382-407.

Phillips, J. M. (1998). Effects of realistic job previews on multiple organizational outcomes: A meta-analysis. Academy of Management Journal, 41(6), 673-690.

Piaget, J. (1926/1960). The child's conception of the world (J. \& A. Tomlinson, Trans.). Totowa, NJ: Littlefield, Adams.

Posthuma, R. A., Morgeson, F. P., \& Campion, M. A. (2002). Beyond employment interview validity: A comprehensive narrative review of recent research and trends over time. Personnel Psychology, 55(1), 1-81.

Preacher, K. J., \& Hayes, A. F. (2008). Contemporary approaches to assessing mediation in communication research. In A. F. Hayes, M. D. Slater, \& L. B. Snyder (Eds.), The Sage sourcebook of advanced data analysis methods for communication research. (pp. 12-54). Thousand Oaks, CA: Sage.

Preacher, K. J., \& Kelley, K. (2011). Effect size measurements for mediation models: Quantitative strategies for communicating interaction effects. Psychological Methods, 16(2), 93-115.

Pretsch, J., Flunger, B., Heckmann, N., \& Schmitt, M. (2013). Done in 60s? Inferring teachers' subjective well-being from thin slices of nonverbal behavior. Social Psychology of Education: An International Journal, 16(3), 421-434. 
Pulakos, E. D., \& Schmitt, N. (1995). Experience-based and situational interview questions: Studies and validity. Personnel Psychology, 48(2), 289-308.

Rajan, M. V., \& Reichelstein, S. (2009). Objective versus subjective indicators of managerial performance. The Accounting Review, 84(1), 209-237.

Richeson, J. A., \& Shelton, J. N. (2005). Brief report: Thin slices of racial bias. Journal of Nonverbal Behavior, 29(1), 75-86.

Rilling, J. K., \& Sanfey, A. G. (2011). The neuroscience of social decision making. Annual Review of Psychology, 62, 23-48.

Reber, A. S (1993). Implicit learning and tacit knowledge. Oxford, UK: Oxford University Press.

Reder, L. M. (1982). Plausibility judgments versus fact retrieval: Alternative strategies for sentence verification. Psychological Review, 89(3), 250-280.

Reyna, V. F., \& Brainerd, C. J. (1995a). Fuzzy-trace theory: An interim synthesis. Learning and Individual Differences, 7(1), 1-75.

Reyna, V. F., \& Brainerd, C. J. (1995b). Fuzzy-trace theory: Some foundational issues. Learning and Individual Differences, 7(2), 145-162.

Reyna, V. F., Brainerd, C. J., \& Connolly, T. (1990). Just the bottom line, please: A fuzzy-trace theory of framing effects in choice. In Fifth International Conference on the Foundation and Application of Utility, Risk, and Decision Theories, Duke University.

Rodell, J. B., \& Colquitt, J. A. (2009). Looking ahead in times of uncertainty: The role of anticipatory justice in an organizational change context. Journal of Applied Psychology, 94(4), 989-1002. 
Rodger, A. (1952). The worthwhileness of the interview. Occupational Psychology, 26(2), 101106.

Russell, B. (1903). The principles of mathematics. Cambridge: Cambridge University Press.

Ryan, R. M., Mimms, v., \& Koestner, R. (1983). Relation of reward contingency and interpersonal context to intrinsic motivation: A review and test using cognitive evaluation theory. Journal of Personality and Social Psychology, 45(4), 736-750.

Ryan, A. M., \& Ployhart, R. E. (2000). Applicants' perceptions of selection procedures and decisions: A critical review and agenda for the future. Journal of Management, 26(3), 556-606.

Rynes, S. L. (1993). Who's selecting whom? Effects of selection practices on applicant attitudes and behavior. In N. Schmitt \& W. C. Borman (Eds.), Personnel selection in organizations (pp. 240-274). San Francisco, CA: Jossey-Bass.

Rynes, S. L., Bretz, R. D., \& Gerhart, B. (1991). The importance of recruitment in job choice: A different way of looking. Personnel Psychology, 44(3), 487-521.

Rynes, S. L., Colbert, A. E., \& Brown, K. G. (2002). HR professions' beliefs about effective human resource practices: Correspondence between research and practice. Human Resource Management, 41(2), 149-174.

Rynes, S. L., \& Gerhart, B. (1990). Interviewer assessments of applicant "fit": An exploratory investigation. Personnel Psychology, 43(1), 13-35. 
Sasso, K. E., \& Strunk, D. R. (2013). Thin slice ratings of client characteristics in intake assessments: Predicting symptom change and dropout in cognitive therapy for depression. Behaviour Research and Therapy, 51(8), 443-450.

Schlenker, B. R. (1980). Impression Management. Monterey, CA: Brooks/Cole.

Schmitt, N. (1976). Social and situational determinants of interview decisions: Implications for the employment interview. Personnel Psychology, 29(1), 79-101.

Schmitt, N. \& Gilliland, S. W. (1992). Test-taking dispositions: A missing link? Journal of Applied Psychology, 77(5), 629-637.

Schneider, B., Smith, D. B., Taylor, S., \& Fleenor, J. (1998). Personality and organizations: A test of the homogeneity of personality hypothesis. Journal of Applied Psychology, 83(3), 462-470.

Schwab, D. P. (1973). Impact of alternative compensation systems on pay valence and instrumentality perceptions. Journal of Applied Psychology, 58(3), 308-312.

Segal, M. (1986). Post-institutionalism in labor economics: The forties and fifties revisited. Industrial \& Labor Relations Review, 39(3), 388-403.

Shallice, T., \& Warrington, E. K. (1970). Independent functioning of verbal memory stores: A neuropsychology study. Quarterly Journal of Experimental Psychology, 22(2), 261-273.

Shannon, B. (1987). On the place of representations in psychology. In J. Bishop, J. Lockheed, \& D. N. Perkins (Eds.), Thinking. Hillsdale, NJ: Erlbaum. 
Shapiro, D. L., \& Kirkman, B. L. (2001). Anticipatory injustice: The consequences of expecting injustice in the workplace. In J. Greenberg \& R. Cropanzano (Eds.), Advances in organizational justice (pp. 152-178). Lexington, MA: New Lexington.

Shiffrin, R. M., \& Schneider, W. (1977). Controlled and automatic human information processing: II. Perceptual learning, automatic attending and general theory. Psychological Review, 84(2), 127-190.

Shotland, A., Alliger, G. M., \& Sales, T. (1998). Face validity in the context of personnel selection: A multimedia approach. International Journal of Selection and Assessment, 6(2), 124-130.

Simmons, J. P., \& Nelson, L. D. (2006). Intuitive confidence: Choosing between intuitive and nonintuitive alternatives. Journal of Experimental Psychology, 135(3), 409-428.

Simon, H. A. (1947). Administrative behavior: A study of decision-making processes in administrative organization. New York, NY: McMillan.

Simon, H. A. (1956). Rational choice and the structure of the environment. Psychological Review, 63(2), 129-138.

Sims, H. P., Jr. \& Lorenzi, P. (1992). The new leadership paradigm: Social learning and cognition in organizations. Newbury Park, CA: Sage.

Skinner, B. F. (1938). The behavior of organisms: An experimental analysis. New York, NY: Appleton-Century. 
Sleesman D. J., Conlon, D. E., McNamara, G., \& Miles, J. E. (2012). Cleaning up the big muddy: A meta-analytic review of the determinants of escalation of commitment. Academy of Management Journal, 55(3), 541-562.

Sloman, S. A. (1996). The empirical case for two systems of reasoning. Psychological Bulletin, 119(1), 3-22.

Smedslund, J. (1969). Psychological diagnostics. Psychological Bulletin, 71(3), 237-248.

Smith, A. (1776/1976). An inquiry into the nature and causes of the wealth of nations. Dunwoody, GA: Norman S. Berg.

Smith, E. R., \& DeCoster, J. (2000). Dual-process models in social and cognitive psychology: Conceptual integration and links to underlying memory systems. Personality and Social Psychology Review, 4(2), 108-131.

Smither, J. W., Reilly, R. R., Millsap, R. E., Pearlman, K., \& Stoffey, R. W. (1993). Applicant reactions to selection procedures. Personnel Psychology, 46(1), 49-76.

Spreitzer, G. M. (1995). Social structural characteristics of psychological empowerment. Academy of Management Journal. 39(2), 483-504.

Staddon, J. E. R., \& Cerutti, D. T. (2003). Operant conditioning. Annual Review of Psychology, 54, 115-144.

Stajkovic, A. D., \& Luthans, F. (1997). A meta-analysis of the effects of organizational behavior modification on task performance, 1975-1995. Academy of Management Journal, 40(5), 1122-1149. 
Stajkovic, A. D., \& Luthans, F. (1998). Self-efficacy and work-related performance: A metaanalysis. Psychological Bulletin, 124(2), 240-261.

Stanovich K. E. (1999). Who is rational? Studies of individual differences and reasoning. Mahwah, NJ: Lawrence Erlbaum Associates

Stasser, G. (1992). Information salience and the discovery of hidden profiles by decision-making groups: A “thought experiment." Organizational Behavior and Human Decision Processes, 52(1), 156-181.

Staw, B. M. (1981). The escalation of commitment to a course of action. Academy of Management Review, 6(4), 577-587.

Stiff, J. B., Miller, G. R., Sleight, C., Mongeau, P. Garlick, R., \& Rogan, R. (1989). Explanations for visual cue primacy in judgments of honesty and deceit. Journal of Personality and Social Psychology, 56(4), 1660-1672.

Stillman, T. F., Maner, J. K., \& Baumeister, R. F. (2010). A thin slice of violence: Distinguishing violent form nonviolent sex offenders at a glance. Evolution and Human Behavior, 31(4), 298-303.

Strack, F., \& Deutsch, R. (2004). Reflective and impulsive determinants of social behavior. Personality and Social Psychology Review, 8(3), 220-247.

Sue-Chan, C., \& Ong, M. (2002). Goal assignment and performance: Assessing the mediating roles of goal commitment and self-efficacy and the moderating role of power distance. Organizational Behavior and Human Decision Processes, 89(2), 1140-1161. 
Sweller, J. (1988). Cognitive load during problem solving: Effects on learning. Cognitive Science, 12(2), 257-285.

Sweller, J., Chandler, P., Tierney, P., \& Cooper, M. (1990). Cognitive load and selective attention as factors in the structuring of technical material. Journal of Experimental Psychology, 119(2), 176-192.

Taylor, P. J., \& Small, B. (2002). Asking applicants what they would do versus what they do: A meta-analytic comparison of situational and past behavior employment interview questions. Journal of Occupational and Organizational Psychology, 75(3), 277-294.

Thompson, V. A. (2009). Dual-process theories: A metacognitive perspective. In J. St. B . T. Evans, \& K. Frankish (Eds.), In two minds: Dual processes and beyond (pp. 171-196). Oxford, UK: Oxford University Press.

Thoresen, C. J., Bradley, J. C., Bliese, P. D., \& Thoresen, J. D. (2004). The big five personality traits and individual job performance growth trajectories in maintenance and transitional job stages. Journal of Applied Psychology, 89(5), 835-853.

Thorndike, E. L. (1905). The elements of psychology. New York, NY: A. g. Seiler.

Toates, F. (2006). A model of the hierarchy of behaviour, cognition, and consciousness. Consciousness and Cognition, 15(1), 75-118.

Tolman, E. C. (1948). Cognitive maps in rats and men. Psychological Review, 55(4), 189-208.

Tom, G., Tong, S. T., \& Hesse, C. (2010). Thick slice and thin slice teaching evaluations. Social Psychology of Education: An International Journal, 13(1), 129-136. 
Topor, D. J., Colarelli, S. M., \& Han, K. (2007). Influences of traits and assessment methods on human resource practitioners' evaluations of job applicants. Journal of Business and Psychology, 21(3), 361-376.

Tosi, H. L., Werner, S., Katz, J. P., \& Gomez-Mejia, L. R. (2000). How much does performance matter? A meta-analysis of CEO pay studies. Journal of Management, 26(2), 301-339.

Tremblay, M. A., Blanchard, C. M., Taylor, S., Pelletier, L. G., \& Villeneuve, M. (2009). Work extrinsic and intrinsic motivation scale: Its value for organizational psychology research. Canadian Journal of Behavioral Science, 41(4), 213-226.

Tsai, C. I., Klayman, J., \& Hastie, R. (2008). Effects of amount of information on judgment accuracy and confidence. Organizational Behavior and Human Decision Processes, 107(2), 97-105.

Tullar, W. L., Mullins, T. W., Caldwell, S. A. (1979). Effects of interview length and applicant quality on interview decision time. Journal of Applied Psychology, 64(4), 669-674.

Tversky, A., \& Kahneman, D. (1974). Judgment under uncertainty: Heuristics and biases. Science, 185(4157), 1124-1131.

Tversky, A., \& Kahneman, D. (1986). Rational choice and the framing of decision. Journal of Business, 59(4), S251-S278.

Ulrich, L., \& Trumbo, D. (1965). The selection interview since 1949. Psychological Bulletin, 63(2), 100-116. 
U.S. Bureau of Labor Statistics (2014). Employer costs for employee compensation summary.

Retrieved from the World Wide Web February 2014 at:

www.bls.gov/news.release/ecec.nr0.htm

Vallacher, R. R., \& Wegner, D. M. (1987). What do people think they're doing? Action identification and human behavior. Psychological Review, 94(1), 3-15.

Van Eerde, W., \& Thierry, H. (1996). Vroom's expectancy models and work-related criteria: A meta-analysis. Journal of Applied Psychology, 81(5), 575-586.

Vessey, I. (1991). Cognitive fit: A theory-based analysis of the graph versus tables literature. Decision Science, 22(2), 219-240.

Vinchur, A. J., Schippmann, J. S., Switzer, F. S., \& Roth, P. L. (1998). A meta-analytic review of predictors of job performance in salespeople. Journal of Applied Psychology, 83(4), 586-597.

Vroom, V. H. (1964). Work and motivation. New York, NY: Wiley

Wagner, R. (1949). The employment interview: A critical summary. Personnel Psychology, 2(1), 17-46.

Wayne, S. J., \& Ferris, G. R. (1990). Influence tactics, affect, and exchange quality in supervisor-subordinate interactions: A laboratory experiment and field study. Journal of Applied Psychology, 75(5), 487-499.

Wayne, S.J., Liden, R.C., Graf, I.K., \& Ferris, G.R. (1997). The role of upward influence tactics in human resource decisions. Personnel Psychology, 50(4), 979-1006. 
Wegner, D. M., \& Bargh, J. A. (1998). Control and automaticity in social life. In D. Gilbert, S. Fiske, \& G. Lindzey (Eds.), Handbook of Social Psychology (4 $4^{\text {th }}$ ed.) (pp. 446-496). Boston, MA: McGraw Hill.

Weirsma, U., \& Latham, G. P. (1986). The practicality of behavioral observation scales, behavioral expectation scales, and trait scales. Personnel Psychology, 39(3), 619-628.

Weisner, W., \& Cronshaw, S. (1988). A meta-analytic investigation of the impact of interview format and degree of structure on the validity of the employment interview. Journal of Occupational Psychology, 61(4), 275-290.

White, H. (1980). A heteroskedasticity-constant covariance matrix estimator and a direct test for heteroskedasticity. Econometrika, 48(4), 817-830.

Whitehead, A. N., \& Russell, B. (1910-1913). Principia mathematica (Vols. 1-3). Cambridge: Cambridge University Press.

Whyte, G. (1989). Groupthink reconsidered. Academy of Management Review. 14(1), 40-56.

Whyte, G. (1993). Escalating commitment in individual and group decision making: A prospect theory approach. Organizational Behavior and Human Decision Processes, 54(3), 430455.

Williams, L. J., \& Hazer, J. T. (1986). Antecedents and consequences of satisfaction and commitment in turnover models: A reanalysis using latent variable structural equation models. Journal of Applied Psychology, 71(2), 219-231.

Wilson, M. (2002). Six views of embodied cognition. Psychonomic Bulletin and Review, 9(4), 625-636. 
Wright, P. M., Lichtenfels, P. A., \& Pursell, E. D. (1989). The structured interview: Additional studies and a meta-analysis. Journal of Occupational Psychology, 62(3), 191-199.

Wright, P. M., \& Boswell, W. R. (2002). Desegregating HRM: A review and synthesis of micro and macro human resource management research. Journal of Management, 28(3), 247276.

Wong, E. M., Ormiston, M. E., \& Tetlock, P. E. (2011). The effects of top management team integrative complexity and decentralized decision making on corporate social performance. Academy of Management Journal, 54(6), 1207-1228.

Wood, R. E., Adkins, P. W. B., \& Bright, J. E. H. (1999). Bonuses, goals, and instrumentality effects. Journal of Applied Psychology, 84(5), 703-720.

Yates, J. F. (1990). Judgment and decision making. Englewood Cliffs, NJ: Prentice-Hall, Inc.

Zenger, T. R., \& Marshall, C. R. (2000). The determinants of incentive intensity in group-based rewards. Academy of Management Journal, 43(2), 149-163. 
Table 1

Dual Processing Terminology Disagreement

\begin{tabular}{|c|c|l|}
\hline System 1 & System 2 & \multicolumn{1}{|c|}{ Reference } \\
\hline Automatic & Controlled & Schneider \& Schiffrin (1977) \\
\hline Heuristic & Systematic & Chaiken (1980) \\
\hline Input Modules & Higher Cognition & Fodor (1983) \\
\hline Heuristic & Analytic & St. B. T. Evans (1989) \\
\hline Implicit & Explicit & Reber (1993) \\
\hline Experiential & Rational & Epstein (1994) \\
\hline Associative & Rule-Based & Sloman (1996) \\
\hline Intuitive & Analytic & Hammond (1996) \\
\hline System 1 & System 2 & Stanovich (1999) \\
\hline Associative & Rule-Based & Smith \& DeCoster (2000) \\
\hline Holistic & Analytic & Nisbett et al (2001) \\
\hline Adaptive Unconscious & Conscious & Wilson (2002) \\
\hline Reflexive & Reflective & Lieberman (2000) \\
\hline Impulsive & Reflective & Strack \& Deustch (2004) \\
\hline Stimulus Bound & Higher Order & Toates (2006) \\
\hline Type 1 Cluster & Type 2 Cluster & St. B. T. Evans (2008) \\
\hline
\end{tabular}


Table 2

Brainerd \& Reyna's (1988) Responses to Memory Probes

\begin{tabular}{c|c|c|c|}
\multicolumn{1}{c|}{} & \multicolumn{2}{c|}{ Retrieval Type } \\
\cline { 2 - 4 } \multicolumn{1}{c|}{ Gist } & Simple & Reconstructive \\
\cline { 2 - 4 } $\begin{array}{c}\text { Information } \\
\text { Specificity }\end{array}$ & $\begin{array}{c}\text { Simple Retrieval of } \\
\text { Gist Traces }\end{array}$ & $\begin{array}{c}\text { Reconstructive } \\
\text { Retrieval of Gist } \\
\text { Traces }\end{array}$ \\
\cline { 2 - 4 } & Verbatim & $\begin{array}{c}\text { Simple Retrieval of } \\
\text { Verbatim Traces }\end{array}$ & $\begin{array}{c}\text { Reconstructive } \\
\text { Retrieval of } \\
\text { Verbatim Traces }\end{array}$ \\
\hline
\end{tabular}


Table 3

Thin Slicing Empirical Review

\begin{tabular}{|c|c|c|c|c|c|}
\hline Author & $\begin{array}{l}\text { Factor Being } \\
\text { Assessed }\end{array}$ & Moderator & $\begin{array}{c}\text { Referent } \\
\text { Comparison }\end{array}$ & Slice Lengths & Results \\
\hline $\begin{array}{c}\text { Ambady \& Rosenthal } \\
1993\end{array}$ & $\begin{array}{c}\text { Teacher } \\
\text { effectiveness }\end{array}$ & None & Thick Slices & $\begin{array}{l}6,15, \& 30 \\
\text { seconds }\end{array}$ & Support for thin slicing validity \\
\hline Ambady 2010 & $\begin{array}{c}\text { Teacher } \\
\text { effectiveness }\end{array}$ & Cognitive load & $\begin{array}{l}\text { Random } \\
\text { Chance }\end{array}$ & 10 seconds & $\begin{array}{l}\text { Cognitive overload decreases } \\
\text { effectiveness of thin slicing }\end{array}$ \\
\hline Ambady et al 1999 & Sexual orientation & $\begin{array}{l}\text { Informational } \\
\text { dynamism }\end{array}$ & $\begin{array}{l}\text { Random } \\
\text { Chance }\end{array}$ & 10 seconds & $\begin{array}{l}\text { Presentation of dynamic information } \\
\text { increases effectiveness of thin slicing }\end{array}$ \\
\hline Ambady \& Gray 2002 & $\begin{array}{l}\text { Teacher } \\
\text { effectiveness and } \\
\text { relationship type }\end{array}$ & Mood & $\begin{array}{l}\text { Random } \\
\text { Chance }\end{array}$ & $\begin{array}{l}2,10, \text { and } 15 \\
\text { seconds }\end{array}$ & Sadness increases accuracy \\
\hline Ambady et al 2006 & $\begin{array}{l}\text { Salesperson } \\
\text { effectiveness }\end{array}$ & None & $\begin{array}{l}\text { Random } \\
\text { Chance }\end{array}$ & 20 seconds & $\begin{array}{l}\text { Customers accurately identified } \\
\text { interpersonal skills, task skills, and } \\
\text { anxiety }\end{array}$ \\
\hline Ames et al 2010 & Big Five traits & $\begin{array}{l}\text { Judgment } \\
\text { confidence }\end{array}$ & $\begin{array}{l}\text { Random } \\
\text { Chance }\end{array}$ & $\begin{array}{l}\text { Photo recognition } \\
\text { and } 60 \text { seconds }\end{array}$ & $\begin{array}{l}\text { Raters have a limited ability to } \\
\text { predict their own accuracy based on } \\
\text { thin slices }\end{array}$ \\
\hline Babad 2005 & Teacher reactions & None & $\begin{array}{l}\text { Random } \\
\text { Chance }\end{array}$ & 10 seconds & Support for thin slicing validity \\
\hline Borkenau et al 2004 & $\begin{array}{l}\text { Personality and } \\
\text { intelligence }\end{array}$ & None & Thick Slices & Varying & Support for thin slicing validity \\
\hline Carney et al 2007 & $\begin{array}{l}\text { Individual } \\
\text { differences }\end{array}$ & Slice length & $\begin{array}{l}\text { Random } \\
\text { Chance }\end{array}$ & Varying & $\begin{array}{l}\text { Length necessary for accuracy } \\
\text { depends on the individual difference } \\
\text { being considered }\end{array}$ \\
\hline $\begin{array}{l}\text { Curhan \& Pentland } \\
2007\end{array}$ & $\begin{array}{l}\text { Negotiation } \\
\text { outcomes }\end{array}$ & None & Thick Slices & 5 minutes & Support for thin slicing validity \\
\hline
\end{tabular}


Table 3 (Cont'd)

Thin Slicing Empirical Review

\begin{tabular}{|c|c|c|c|c|c|}
\hline Author & $\begin{array}{l}\text { Factor Being } \\
\text { Assessed }\end{array}$ & Moderator & $\begin{array}{c}\text { Referent } \\
\text { Comparison }\end{array}$ & Slice Lengths & Results \\
\hline Fowler et al 2009 & $\begin{array}{l}\text { Psychopathy } \\
\text { levels }\end{array}$ & None & $\begin{array}{l}\text { Random } \\
\text { Chance }\end{array}$ & $\begin{array}{l}5,10, \text { and } 20 \\
\text { seconds }\end{array}$ & Support for thin slicing validity \\
\hline Friedman et al 2007 & $\begin{array}{l}\text { Personality } \\
\text { disorders }\end{array}$ & None & $\begin{array}{l}\text { Random } \\
\text { Chance }\end{array}$ & 30 seconds & Support for thin slicing validity \\
\hline Holleran et al 2009 & Daily behavior & Proximity to actor & $\begin{array}{l}\text { Random } \\
\text { Chance }\end{array}$ & 2.5 minutes & $\begin{array}{l}\text { Strangers are as accurate as } \\
\text { acquaintances and targets themselves }\end{array}$ \\
\hline Kraus \& Keltner 2009 & $\begin{array}{c}\text { Socioeconomic } \\
\text { status }\end{array}$ & None & $\begin{array}{l}\text { Random } \\
\text { Chance }\end{array}$ & 60 seconds & Support for thin slicing validity \\
\hline Murphy 2005 & Behavioral coding & None & Thick Slices & 1 minute & Support for thin slicing validity \\
\hline Oltmanns et al 2004 & $\begin{array}{l}\text { Personality } \\
\text { disorders }\end{array}$ & None & $\begin{array}{l}\text { Random } \\
\text { Chance }\end{array}$ & 30 seconds & Support for thin slicing validity \\
\hline Pretsch et al 2013 & $\begin{array}{l}\text { Teacher well- } \\
\text { being }\end{array}$ & None & $\begin{array}{l}\text { Random } \\
\text { Chance }\end{array}$ & 60 seconds & Support for thin slicing validity \\
\hline $\begin{array}{l}\text { Richeson \& Shelton } \\
2005\end{array}$ & Racial bias & None & $\begin{array}{l}\text { Random } \\
\text { Chance }\end{array}$ & 20 seconds & $\begin{array}{l}\text { Racial bias occurs in thin slices when } \\
\text { only observing nonverbal behavior }\end{array}$ \\
\hline Sasso \& Strunk 2013 & $\begin{array}{c}\text { Therapy } \\
\text { effectiveness }\end{array}$ & None & $\begin{array}{l}\text { Random } \\
\text { Chance }\end{array}$ & Less than 1 minute & Support for thin slicing validity \\
\hline Stillman et al 2010 & $\begin{array}{l}\text { Propensity for } \\
\text { violence }\end{array}$ & None & $\begin{array}{c}\text { Random } \\
\text { Chance }\end{array}$ & Photo recognition & Support for thin slicing validity \\
\hline Tom et al 2010 & $\begin{array}{l}\text { Student-based } \\
\text { teaching } \\
\text { evaluations }\end{array}$ & None & Thick Slices & 30 seconds & $\begin{array}{l}\text { Mixed support. Information gained } \\
\text { during thicker slices }\end{array}$ \\
\hline
\end{tabular}


Table 4

Means, Standard Deviations, and Intercorrelations Among Study Variables

\begin{tabular}{|c|c|c|c|c|c|c|c|c|c|c|c|c|c|c|}
\hline & Variable & $M$ & $S D$ & 1 & 2 & 3 & 4 & 5 & 6 & 7 & 8 & 9 & 10 & 11 \\
\hline 1. & Gender Congruence & .49 & .50 & --- & & & & & & & & & & \\
\hline 2. & Race Congruence & .18 & .38 & -.047 & -- & & & & & & & & & \\
\hline 3. & GPA & 3.70 & .80 & -.063 & -.012 & -- & & & & & & & & \\
\hline 4. & Work Experience & 2.30 & .71 & .009 & $-.250 * *$ & $-.157 *$ & --- & & & & & & & \\
\hline 5. & Compensation Condition & .50 & .50 & .030 & -.065 & -.037 & $.151 *$ & -- & & & & & & \\
\hline 6. & T1 Employability & 23.66 & 5.19 & .070 & -.086 & .128 & .016 & $.146^{*}$ & -- & & & & & \\
\hline 7. & T1 Confidence & 16.25 & 3.97 & .039 & -.010 & $.193 * *$ & .031 & .053 & $.488^{* *}$ & -- & & & & \\
\hline 8. & T2 Employability & 22.94 & 6.49 & -.005 & -.101 & $.206^{* *}$ & -.054 & $.151 *$ & $.227 * *$ & .128 & -- & & & \\
\hline 9. & T2 Confidence & 18.20 & 3.58 & -.011 & -.071 & $.157 *$ & -.057 & .009 & .068 & $.297 * *$ & $.447 * *$ & -- & & \\
\hline 10. & Interview Score & 20.50 & 4.97 & -.076 & -.107 & $.181 * *$ & .096 & .110 & .025 & -.061 & $.461 * *$ & $.176^{* *}$ & -- & \\
\hline 11. & T3 Employability & 22.57 & 7.30 & .023 & -.115 & $.178^{* *}$ & .028 & $.154 *$ & .099 & -.058 & $.713 * *$ & $.328 * *$ & $.666^{* *}$ & - \\
\hline
\end{tabular}


Table 5

Baseline Model

Structural Path Results

\begin{tabular}{lrc}
\hline \multicolumn{1}{c}{ Structural Paths } & $\beta$ & $R^{2}$ \\
\hline Gender Congruence $\rightarrow$ Second Impression & -.037 & .001 \\
Race Congruence $\rightarrow$ Second Impression & -.016 & .000 \\
GPA $\rightarrow$ Second Impression & $.183^{* *}$ & .033 \\
Work Experience $\rightarrow$ Second Impression & .054 & .003 \\
Gender Congruence $\rightarrow$ Final Impression & .050 & .003 \\
Race Congruence $\rightarrow$ Final Impression & -.019 & .000 \\
GPA $\rightarrow$ Final Impression & -.016 & .000 \\
Work Experience $\rightarrow$ Final Impression & -.013 & .000 \\
Initial Impression $\rightarrow$ Second Impression & -.010 & 0.00 \\
Second Impression $\rightarrow$ Interview Score & $.589 * * *$ & 0.347 \\
Second Impression $\rightarrow$ Final Impression & $.346^{* * *}$ & 0.120 \\
Interview Score $\rightarrow$ Final Impressions & $.674 * * *$ & 0.454
\end{tabular}

Fit Statistics: $X^{2}=14.625^{*}, d f=7, \mathrm{CFI}=.976, \mathrm{RMSEA}=.069, \mathrm{SRMR}=.049$

$$
\mathrm{p}<.05 * \mathrm{p}<.01 * * \mathrm{p}<.001 * * *
$$


Table 6

Hypothesis 7 Constrained Model

Structural Path Results

\begin{tabular}{lrc}
\hline \multicolumn{1}{c}{ Structural Paths } & $\beta$ & $R^{2}$ \\
\hline Gender Congruence $\rightarrow$ Second Impression & -.037 & .001 \\
Race Congruence $\rightarrow$ Second Impression & -.016 & .000 \\
GPA $\rightarrow$ Second Impression & $.183^{* *}$ & .033 \\
Work Experience $\rightarrow$ Second Impression & .054 & .003 \\
Gender Congruence $\rightarrow$ Final Impression & .068 & .005 \\
Race Congruence $\rightarrow$ Final Impression & -.011 & .000 \\
GPA $\rightarrow$ Final Impression & -.108 & .012 \\
Work Experience $\rightarrow$ Final Impression & -.040 & .002 \\
Initial Impression $\rightarrow$ Second Impression & .047 & .002 \\
Second Impression $\rightarrow$ Interview Score & $.589 * * *$ & .347 \\
Second Impression $\rightarrow$ Final Impression & $.845^{* * *}$ & .714 \\
Interview Score $\rightarrow$ Final Impressions & $.674 * * *$ & .454
\end{tabular}

Fit Statistics: $X^{2}=16.710^{*}, d f=8, \mathrm{CFI}=.972, \mathrm{RMSEA}=.069, \mathrm{SRMR}=.049$

$$
\mathrm{p}<.05 * \mathrm{p}<.01 * * \mathrm{p}<.001 * * *
$$


Table 7

Sattora-Bentler Scaled X² Difference Test for Hypothesis 7

\begin{tabular}{|c|c|}
\hline Baseline $X^{2}$ & 14.625 \\
\hline Baseline Scaled $X^{2}$ & 14.630 \\
\hline Baseline $d f$ & 7 \\
\hline Baseline Scaling Correction Factor & 1.2898 \\
\hline Constrained $X^{2}$ & 16.710 \\
\hline Constrained Scaled $X^{2}$ & 16.711 \\
\hline Constrained $d f$ & 1.1288 \\
\hline Constrained Scaling Correction Factor & 2.085 \\
\hline Scaled $X^{2}$ Difference & .149 \\
\hline P-Value & \\
\hline
\end{tabular}


Table 8

First Impression $\rightarrow$ Second Impression Confidence Moderation (H8a)

Huber-White Analysis Results

\begin{tabular}{lc}
\hline \multicolumn{1}{c}{ Structural Paths } & B \\
\hline Gender Congruence $\rightarrow$ Second Impression & -.265 \\
Race Congruence $\rightarrow$ Second Impression & -1.640 \\
GPA $\rightarrow$ Second Impression & $1.430^{* *}$ \\
Work Experience $\rightarrow$ Second Impression & -.564 \\
First Impression $\rightarrow$ Second Impression & $.365^{* *}$ \\
Confidence in First Impression $\rightarrow$ Second Impression & -.123 \\
First Impression x Confidence $\rightarrow$ Second Impression & $.051^{* *}$ \\
\hline
\end{tabular}

$$
\mathrm{p}<.05^{*} \mathrm{p}<.01 * * \mathrm{p}<.001 * * *
$$


Table 9

Second Impression $\rightarrow$ Interview Score Confidence Moderation (H8b)

Huber-White Analysis Results

\begin{tabular}{lc}
\hline \multicolumn{1}{c}{ Structural Paths } & $\mathrm{B}$ \\
\hline Gender Congruence $\rightarrow$ Interview Score & -.684 \\
Race Congruence $\rightarrow$ Interview Score & -.322 \\
GPA $\rightarrow$ Interview Score & $.784^{*}$ \\
Work Experience $\rightarrow$ Interview Score & $1.092^{*}$ \\
Second Impression $\rightarrow$ Interview Score & $.450^{* * *}$ \\
Confidence in Second Impression $\rightarrow$ Interview Score & -.105 \\
Second Impression x Confidence $\rightarrow$ Interview Score & .007 \\
\hline
\end{tabular}

$$
\mathrm{p}<.05^{*} \mathrm{p}<.01 * * \mathrm{p}<.001 * * *
$$


Table 10

Second Impression $\rightarrow$ Final Impression Confidence Moderation (H8c)

Huber-White Analysis Results

\begin{tabular}{lc}
\hline \multicolumn{1}{c}{ Structural Paths } & B \\
\hline Gender Congruence $\rightarrow$ Final Impression & .364 \\
Race Congruence $\rightarrow$ Final Impression & -.431 \\
GPA $\rightarrow$ Final Impression & .365 \\
Work Experience $\rightarrow$ Final Impression & .702 \\
Second Impression $\rightarrow$ Final Impression & $.827^{* * *}$ \\
Confidence in Second Impression $\rightarrow$ Final Impression & -.014 \\
Second Impression x Confidence $\rightarrow$ Final Impression & .010 \\
\hline
\end{tabular}

$$
\mathrm{p}<.05 * \mathrm{p}<.01 * * \mathrm{p}<.001 * * *
$$


Table 11

Compensation Baseline

Structural Path Results

\begin{tabular}{lcc}
\hline \multicolumn{1}{c}{ Structural Paths } & $\beta$ & $R^{2}$ \\
\hline Gender Congruence $\rightarrow$ Second Impression & .030 & .001 \\
Race Congruence $\rightarrow$ Second Impression & -.050 & .003 \\
GPA $\rightarrow$ Second Impression & .202 & .041 \\
Work Experience $\rightarrow$ Second Impression & .035 & .001 \\
Gender Congruence $\rightarrow$ Final Impression & .110 & .012 \\
Race Congruence $\rightarrow$ Final Impression & -.042 & .002 \\
GPA $\rightarrow$ Final Impression & -.093 & .009 \\
Work Experience $\rightarrow$ Final Impression & -.093 & .009 \\
Initial Impression $\rightarrow$ Second Impression & -.020 & .000 \\
Second Impression $\rightarrow$ Interview Score & $.679 * * *$ & .461 \\
Second Impression $\rightarrow$ Final Impression & $.384 * *$ & .147 \\
Interview Score $\rightarrow$ Final Impressions & $.623 * * *$ & .388
\end{tabular}

Fit Statistics: $X^{2}=22.577, d f=14, \mathrm{CFI}=.974, \mathrm{RMSEA}=.073, \mathrm{SRMR}=.061$

$$
\mathrm{p}<.05^{*} \mathrm{p}<.01 * * \mathrm{p}<.001 * * *
$$


Table 12

Full Compensation Moderation

Structural Path Results

\begin{tabular}{lrc}
\hline \multicolumn{1}{c}{ Structural Paths } & $\beta$ & $R^{2}$ \\
\hline Gender Congruence $\rightarrow$ Second Impression & .031 & .001 \\
Race Congruence $\rightarrow$ Second Impression & -.048 & .002 \\
GPA $\rightarrow$ Second Impression & .202 & .041 \\
Work Experience $\rightarrow$ Second Impression & .036 & .001 \\
Gender Congruence $\rightarrow$ Final Impression & .109 & .012 \\
Race Congruence $\rightarrow$ Final Impression & -.040 & .002 \\
GPA $\rightarrow$ Final Impression & -.100 & .010 \\
Work Experience $\rightarrow$ Final Impression & -.094 & .009 \\
Initial Impression $\rightarrow$ Second Impression & -.012 & .000 \\
Second Impression $\rightarrow$ Interview Score & $.662^{* * *}$ & .438 \\
Second Impression $\rightarrow$ Final Impression & $.424 *$ & .180 \\
Interview Score $\rightarrow$ Final Impressions & $.608 * * *$ & .370
\end{tabular}

Fit Statistics: $X^{2}=25.895, d f=17, \mathrm{CFI}=.973, \mathrm{RMSEA}=.068, \mathrm{SRMR}=.061$

$$
\mathrm{p}<.05 * \mathrm{p}<.01 * * \mathrm{p}<.001 * * *
$$


Table 13

Sattora-Bentler Scaled $X^{2}$ Difference Test for Full Compensation Moderation

\begin{tabular}{|c|c|}
\hline Baseline $X^{2}$ & 22.577 \\
\hline Baseline $d f$ & 14 \\
\hline Baseline Scaling Correction Factor & 1.1855 \\
\hline Constrained $X^{2}$ & 25.895 \\
\hline Constrained $d f$ & 17 \\
\hline Constrained Scaling Correction Factor & 1.0439 \\
\hline Scaled $X^{2}$ Difference & 3.318 \\
\hline P-Value & .345 \\
\hline
\end{tabular}


Table 14

Initial Impressions to Second Impressions Compensation Moderation (H9a)

Structural Path Results

\begin{tabular}{lrc}
\hline \multicolumn{1}{c}{ Structural Paths } & $\beta$ & $R^{2}$ \\
\hline Gender Congruence $\rightarrow$ Second Impression & .030 & .001 \\
Race Congruence $\rightarrow$ Second Impression & -.050 & .003 \\
GPA $\rightarrow$ Second Impression & .202 & .041 \\
Work Experience $\rightarrow$ Second Impression & .035 & .001 \\
Gender Congruence $\rightarrow$ Final Impression & .109 & .012 \\
Race Congruence $\rightarrow$ Final Impression & -.041 & .002 \\
GPA $\rightarrow$ Final Impression & -.096 & .009 \\
Work Experience $\rightarrow$ Final Impression & -.093 & .009 \\
Initial Impression $\rightarrow$ Second Impression & -.010 & .000 \\
Second Impression $\rightarrow$ Interview Score & $.679 * * *$ & .461 \\
Second Impression $\rightarrow$ Final Impression & .399 & .159 \\
Interview Score $\rightarrow$ Final Impressions & $.618 * * *$ & .382
\end{tabular}

Fit Statistics: $X^{2}=24.251, d f=15, \mathrm{CFI}=.972, \mathrm{RMSEA}=.073, \mathrm{SRMR}=.061$

$$
\mathrm{p}<.05 * \mathrm{p}<.01 * * \mathrm{p}<.001 * * *
$$


Table 15

Sattora-Bentler Scaled $X^{2}$ Difference Test for Initial Impressions to Second Impressions (H9a) Compensation Moderation

\begin{tabular}{|c|c|}
\hline Baseline $X^{2}$ & 22.577 \\
\hline Baseline $d f$ & 14 \\
\hline Baseline Scaling Correction Factor & 1.1855 \\
\hline Constrained $X^{2}$ & 24.251 \\
\hline Constrained $d f$ & 15 \\
\hline Constrained Scaling Correction Factor & 1.1037 \\
\hline Scaled $X^{2}$ Difference & 1.4121 \\
\hline P-Value & .235 \\
\hline
\end{tabular}


Table 16

Second Impressions to Interview Score Compensation Moderation (H9b)

Structural Path Results

\begin{tabular}{lcc}
\hline \multicolumn{1}{c}{ Structural Paths } & $\beta$ & $R^{2}$ \\
\hline Gender Congruence $\rightarrow$ Second Impression & .031 & .001 \\
Race Congruence $\rightarrow$ Second Impression & -.048 & .002 \\
GPA $\rightarrow$ Second Impression & .202 & .041 \\
Work Experience $\rightarrow$ Second Impression & .036 & .001 \\
Gender Congruence $\rightarrow$ Final Impression & .107 & .011 \\
Race Congruence $\rightarrow$ Final Impression & -.040 & .002 \\
GPA $\rightarrow$ Final Impression & -.102 & .010 \\
Work Experience $\rightarrow$ Final Impression & -.094 & .009 \\
Initial Impression $\rightarrow$ Second Impression & -.032 & .001 \\
Second Impression $\rightarrow$ Interview Score & $.662^{* * *}$ & .438 \\
Second Impression $\rightarrow$ Final Impression & $.435^{* *}$ & .189 \\
Interview Score $\rightarrow$ Final Impressions & $.605^{* * *}$ & .366
\end{tabular}

Fit Statistics: $X^{2}=22.852, d f=15, \mathrm{CFI}=.976, \mathrm{RMSEA}=.068, \mathrm{SRMR}=.061$

$$
\mathrm{p}<.05 * \mathrm{p}<.01 * * \mathrm{p}<.001 * * *
$$


Table 17

Sattora-Bentler Scaled $X^{2}$ Difference Test for Second Impressions to Interview Score (H9b)

Compensation Moderation

\begin{tabular}{|c|c|}
\hline Baseline $X^{2}$ & 22.577 \\
\hline Baseline $d f$ & 14 \\
\hline Baseline Scaling Correction Factor & 1.1855 \\
\hline Constrained $X^{2}$ & 22.852 \\
\hline Constrained $d f$ & 15 \\
\hline Constrained Scaling Correction Factor & 1.1829 \\
\hline Scaled $X^{2}$ Difference & .2399 \\
\hline P-Value & .624 \\
\hline
\end{tabular}


Table 18

Second Impressions to Final Impressions Compensation Moderation (H9c)

Structural Path Results

\begin{tabular}{lcc}
\hline \multicolumn{1}{c}{ Structural Paths } & $\beta$ & $R^{2}$ \\
\hline Gender Congruence $\rightarrow$ Second Impression & .030 & .001 \\
Race Congruence $\rightarrow$ Second Impression & -.049 & .002 \\
GPA $\rightarrow$ Second Impression & .202 & .041 \\
Work Experience $\rightarrow$ Second Impression & .035 & .001 \\
Gender Congruence $\rightarrow$ Final Impression & .111 & .012 \\
Race Congruence $\rightarrow$ Final Impression & -.043 & .002 \\
GPA $\rightarrow$ Final Impression & -.090 & .008 \\
Work Experience $\rightarrow$ Final Impression & -.093 & .009 \\
Initial Impression $\rightarrow$ Second Impression & -.026 & .001 \\
Second Impression $\rightarrow$ Interview Score & $.679 * * *$ & .416 \\
Second Impression $\rightarrow$ Final Impression & .369 & .136 \\
Interview Score $\rightarrow$ Final Impressions & $.627 * * *$ & .393
\end{tabular}

Fit Statistics: $X^{2}=24.198, d f=15, \mathrm{CFI}=.972, \mathrm{RMSEA}=.073, \mathrm{SRMR}=.061$

$$
\mathrm{p}<.05 * \mathrm{p}<.01 * * \mathrm{p}<.001 * * *
$$


Table 19

Sattora-Bentler Scaled $X^{2}$ Difference Test for Second Impressions to Final Impressions (H9c)

Compensation Moderation

\begin{tabular}{|c|c|}
\hline Baseline $X^{2}$ & 22.577 \\
\hline Baseline $d f$ & 14 \\
\hline Baseline Scaling Correction Factor & 1.1855 \\
\hline Constrained $X^{2}$ & 24.198 \\
\hline Constrained $d f$ & 15 \\
\hline Constrained Scaling Correction Factor & 1.1061 \\
\hline Scaled $X^{2}$ Difference & 1.367 \\
\hline P-Value & .242 \\
\hline
\end{tabular}


Table 20

Effect Sizes for the Indirect Effect of Second Impressions on Final Impressions through Interview

Scores

\begin{tabular}{|c|c|c|c|c|}
\hline Name & Type & Estimate & $\begin{array}{c}95 \% \\
\text { CI } \\
\text { Lower }\end{array}$ & $\begin{array}{c}95 \% \\
\text { CI } \\
\text { Upper }\end{array}$ \\
\hline Index of Mediation & Ratio & .197 & .129 & .259 \\
\hline Shared over Simple Effects & Ratio & .588 & .427 & .708 \\
\hline Index of Variance Explained & Variance & .299 & .199 & .386 \\
\hline Explained & Residual-based & .199 & .134 & .267 \\
\hline Proportion of Maximum Possible & Ratio & .250 & .167 & .317 \\
\hline Indirect Effect & & & & \\
\hline
\end{tabular}


Table 21

Manipulation Check T-Test Results

\begin{tabular}{|c|c|c|c|c|c|c|}
\hline Time & Scale & $\begin{array}{c}\text { Incentive } \\
\text { Condition } \\
\text { Mean } \\
\end{array}$ & $\begin{array}{c}\text { Incentive } \\
\text { Condition SD } \\
\end{array}$ & $\begin{array}{c}\text { Fixed } \\
\text { Condition } \\
\text { Mean } \\
\end{array}$ & $\begin{array}{c}\text { Fixed } \\
\text { Condition SD }\end{array}$ & $\begin{array}{c}\mathrm{P}- \\
\text { Value }\end{array}$ \\
\hline \multirow{5}{*}{ 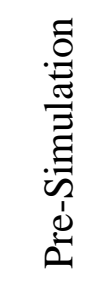 } & Felt Accountability & 42.29 & 6.70 & 36.43 & 5.33 & .017 \\
\hline & Impression Management & 30.86 & 7.69 & 33.29 & 7.36 & .401 \\
\hline & Identified Regulation & 16.29 & 2.84 & 16.14 & 2.91 & .896 \\
\hline & External Regulation & 15.36 & 3.18 & 15.43 & 3.82 & .957 \\
\hline & Intrinsic Motivation & 17.21 & 3.21 & 17.07 & 2.27 & .893 \\
\hline \multirow{5}{*}{ 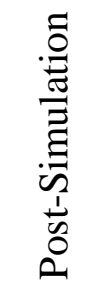 } & Felt Accountability & 37.93 & 8.77 & 36.92 & 5.63 & .734 \\
\hline & Impression Management & 31.21 & 6.23 & 35.33 & 6.37 & .109 \\
\hline & Identified Regulation & 14.79 & 3.12 & 14.67 & 3.50 & .928 \\
\hline & External Regulation & 15.43 & 2.98 & 14.08 & 2.68 & .241 \\
\hline & Intrinsic Motivation & 15.86 & 3.63 & 15.42 & 3.23 & .749 \\
\hline
\end{tabular}


Table 22

Hypothesis Results

\begin{tabular}{|c|c|}
\hline Hypothesized Relationship & Finding \\
\hline $\begin{array}{l}\text { H1: Initial impressions formed at the career fair will positively impact second } \\
\text { impressions formed at the beginning of the interview. }\end{array}$ & $\begin{array}{c}\text { Not } \\
\text { Supported }\end{array}$ \\
\hline $\begin{array}{l}\text { H2: Second impressions of employability formed at the beginning of the } \\
\text { interview will positively impact the applicant's structured interview score. }\end{array}$ & Supported \\
\hline $\begin{array}{l}\text { H3: Second impressions of employability formed at the beginning of the } \\
\text { interview will positively predict final impressions of employability. }\end{array}$ & Supported \\
\hline $\begin{array}{l}\text { H4: Second impressions of employability formed at the beginning of the } \\
\text { interview will mediate the relationship between initial impressions formed at } \\
\text { the career fair and final impressions of employability. }\end{array}$ & Supported \\
\hline $\begin{array}{l}\text { H5: Structured interview scores will positively predict final impressions of } \\
\text { employability. }\end{array}$ & Supported \\
\hline $\begin{array}{l}\text { H6: Structured interview scores will mediate the positive relationship } \\
\text { between second impressions formed at the beginning of the interview and } \\
\text { final impressions of employability. }\end{array}$ & Supported \\
\hline $\begin{array}{l}\text { H7: The direct effect of second impressions of employability formed at the } \\
\text { beginning of the interview on final ratings of employability will be } \\
\text { significantly stronger than the direct effect of structured interview scores on } \\
\text { final ratings of employability. }\end{array}$ & $\begin{array}{l}\text { Not } \\
\text { Supported }\end{array}$ \\
\hline $\begin{array}{l}\text { H8a: Confidence in employability ratings after developing an initial } \\
\text { impression formed at the career fair will moderate the relationship between } \\
\text { initial impressions formed at the career fair and second impressions formed } \\
\text { at the beginning of the interview, such that the relationship between initial } \\
\text { impressions formed at the career fair and second impressions formed at the } \\
\text { beginning of the interview will be stronger as confidence in initial } \\
\text { impressions formed at the career fair increases. }\end{array}$ & Supported \\
\hline $\begin{array}{l}\text { H8b: Confidence in second impressions formed at the beginning of the } \\
\text { interview will moderate the relationship between second impressions formed } \\
\text { at the beginning of the interview and structured interview scores, such that } \\
\text { the relationship between second impressions formed at the beginning of the } \\
\text { interview and structured interview scores is stronger as confidence in second } \\
\text { impressions formed at the beginning of the interview increases. }\end{array}$ & $\begin{array}{l}\text { Not } \\
\text { Supported }\end{array}$ \\
\hline $\begin{array}{l}\text { H8c: Confidence in second impressions formed at the beginning of the } \\
\text { interview will moderate the relationship between second impressions formed } \\
\text { at the beginning of the interview and final impressions of employability, such } \\
\text { that the relationship between second impressions formed at the beginning of } \\
\text { the interview and final impressions is stronger as confidence in second } \\
\text { impressions formed at the beginning of the interview increases. }\end{array}$ & $\begin{array}{l}\text { Not } \\
\text { Supported }\end{array}$ \\
\hline
\end{tabular}


Table 22 (Cont'd)

Hypothesis Results

\begin{tabular}{|c|c|}
\hline Hypothesized Relationship & Finding \\
\hline $\begin{array}{l}\text { H9a: Recruiter incentive compensation will moderate the relationship } \\
\text { between initial impressions formed at the career fair and second impressions } \\
\text { formed at the beginning of the interview such that the relationship between } \\
\text { initial impressions formed at the career fair and second impressions formed } \\
\text { at the beginning of the interview will be stronger under a pay-for- } \\
\text { performance condition than a fixed compensation condition. }\end{array}$ & $\begin{array}{c}\text { Not } \\
\text { Supported }\end{array}$ \\
\hline $\begin{array}{l}\text { H9b: Incentive compensation will moderate the relationship between second } \\
\text { impressions formed at the beginning of the interview and structured interview } \\
\text { scores such that the relationship between second impressions formed at the } \\
\text { beginning of the interview and structured interview scores will be stronger } \\
\text { under a pay-for-performance condition than a fixed compensation condition. }\end{array}$ & $\begin{array}{c}\text { Not } \\
\text { Supported }\end{array}$ \\
\hline $\begin{array}{l}\text { H9c: Recruiter incentive compensation will moderate the relationship } \\
\text { between second impressions formed at the beginning of the interview and } \\
\text { final impressions of employability such that the relationship between second } \\
\text { impressions formed at the beginning of the interview and final impressions of } \\
\text { employability will be stronger under a pay-for-performance condition than a } \\
\text { fixed compensation condition. }\end{array}$ & $\begin{array}{c}\text { Not } \\
\text { Supported }\end{array}$ \\
\hline
\end{tabular}




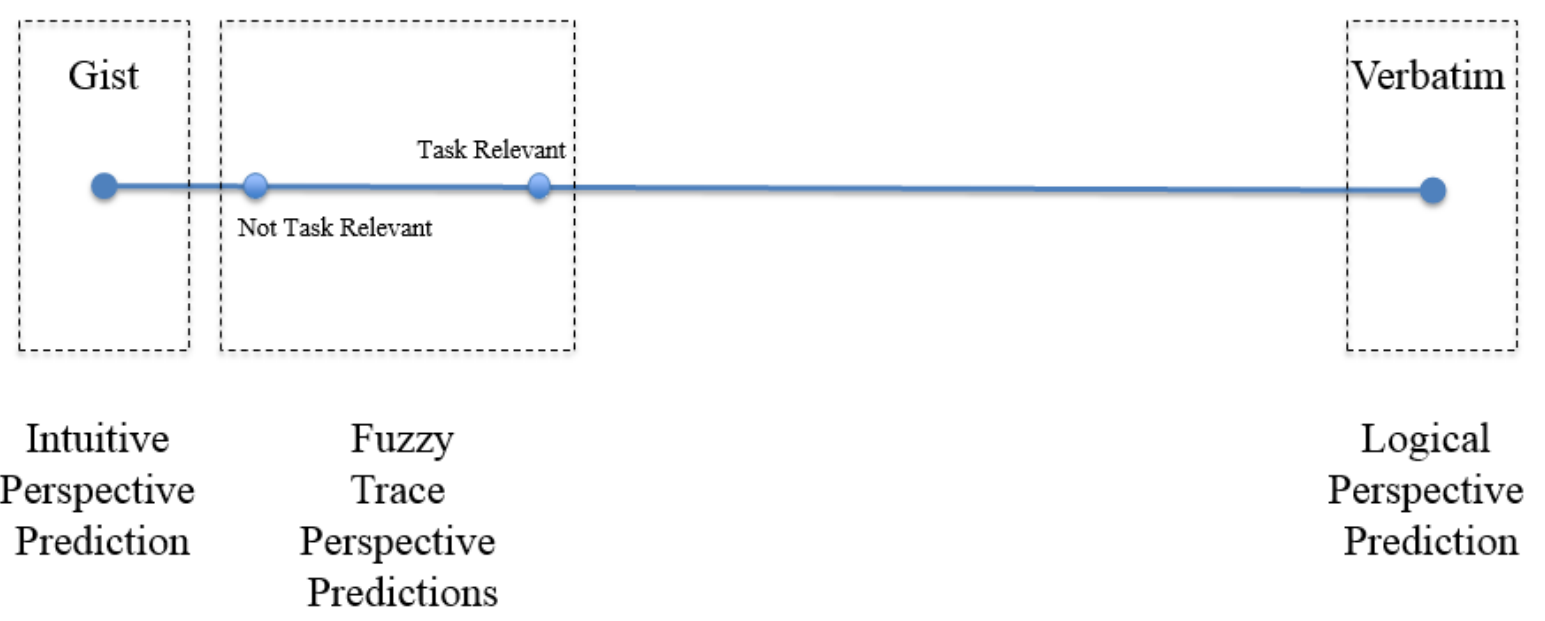

Figure 1. Conceptual Continuum of Information Processing. Intuitive and logical perspectives suggest that processing occurs at the gist and verbatim poles of the continuum respectively, while fuzzy trace suggests that processing occurs toward the gist end with task relevant processing further toward the verbatim end than processing that is not task relevant. 


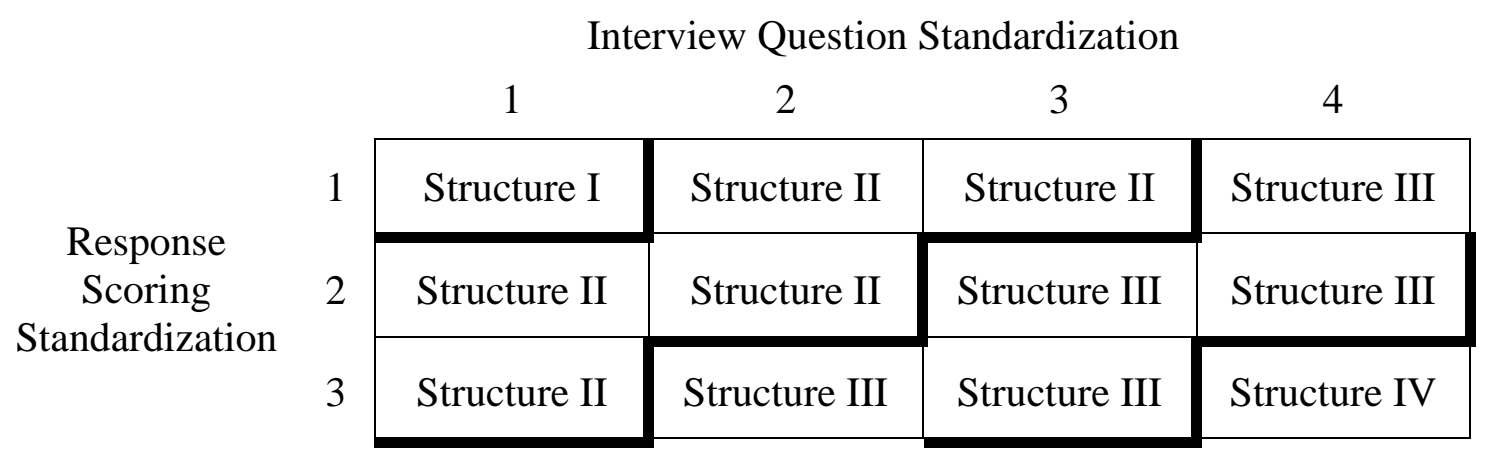

Figure 2. Huffcutt \& Arthur's (1994) Interview Structure Classification Nomenclature 
Individual Effort

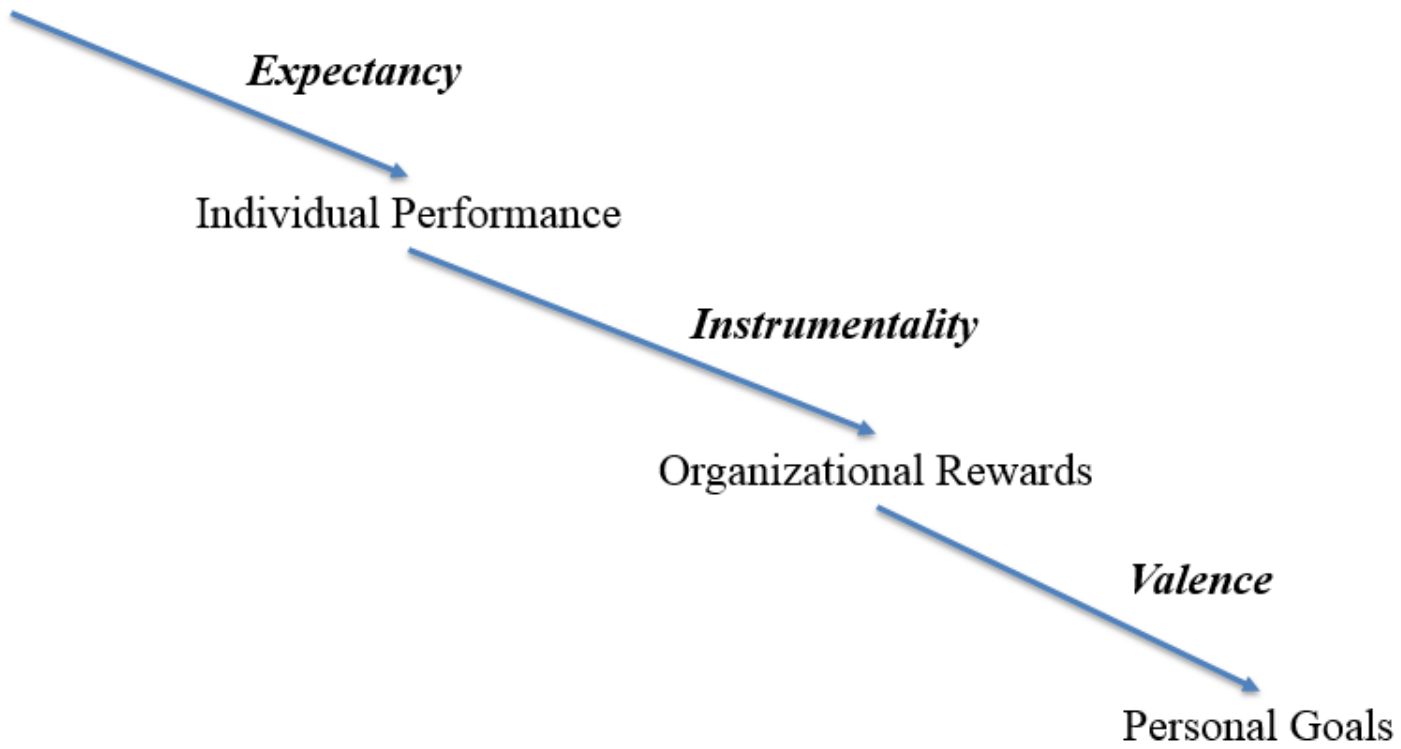

Figure 3. Expectancy Theory Flowchart 

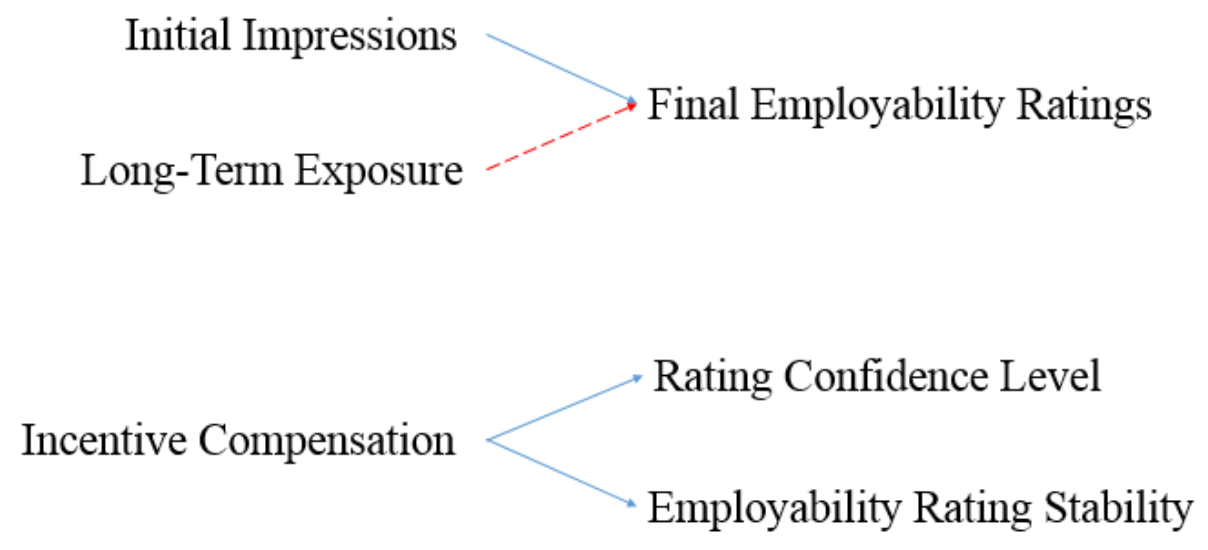

Figure 4. Theoretical Model 


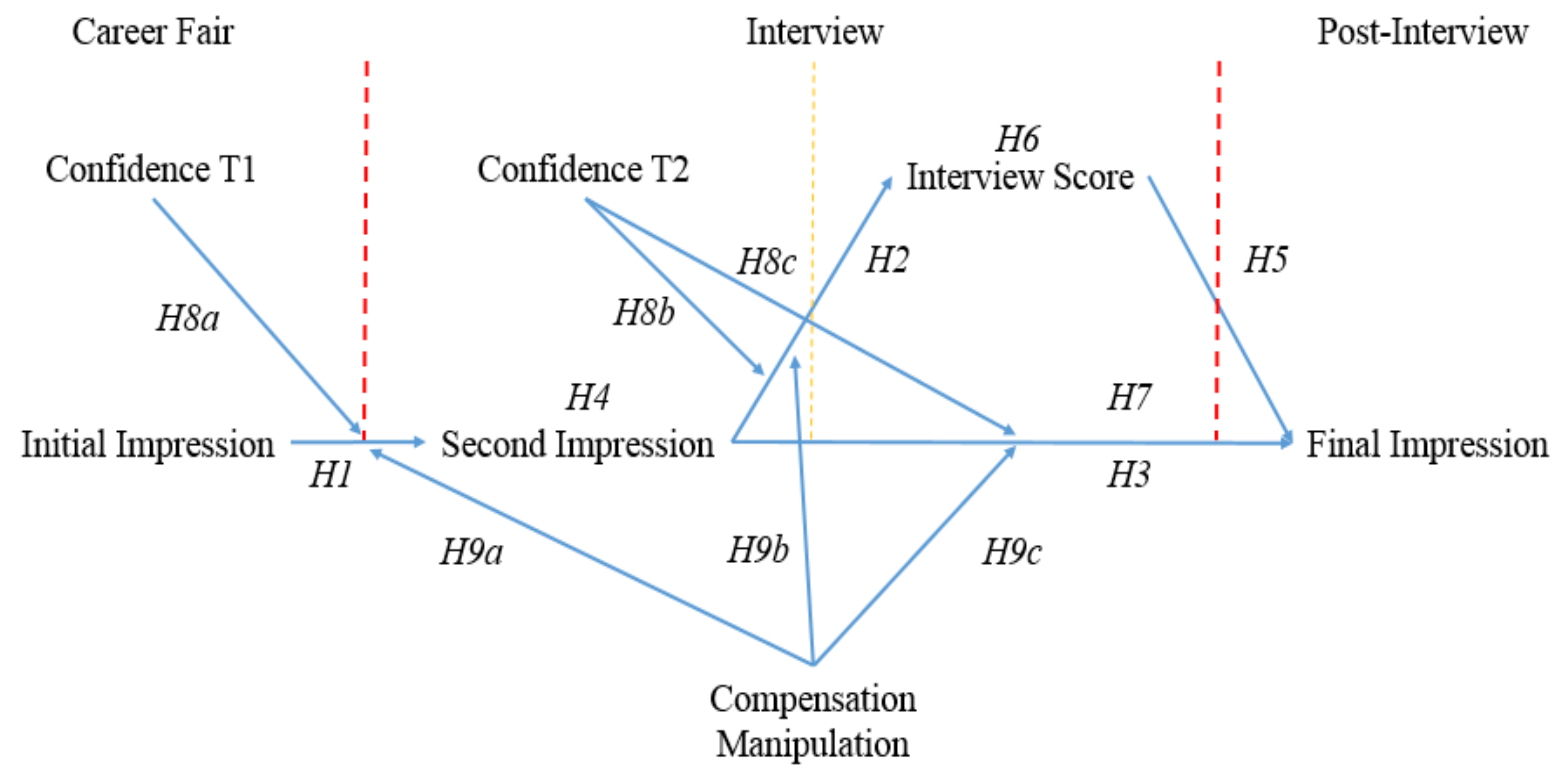

Figure 5. Overall Model 


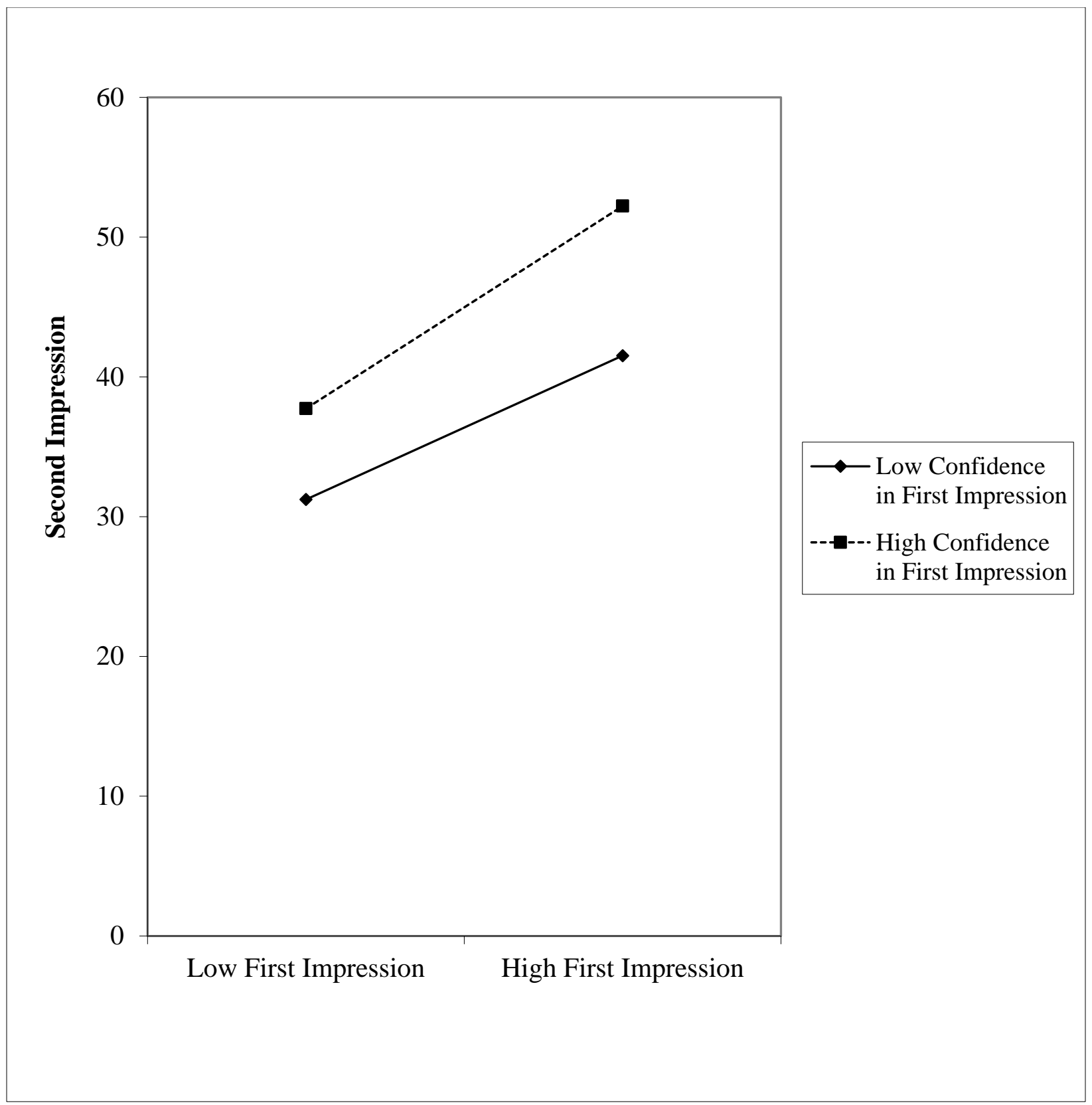

Figure 6. First Impression x Confidence Interaction Plot (H8a) 
$\begin{array}{lll}\text { Career Fair } & \text { Interview } & \text { Post-Interview }\end{array}$

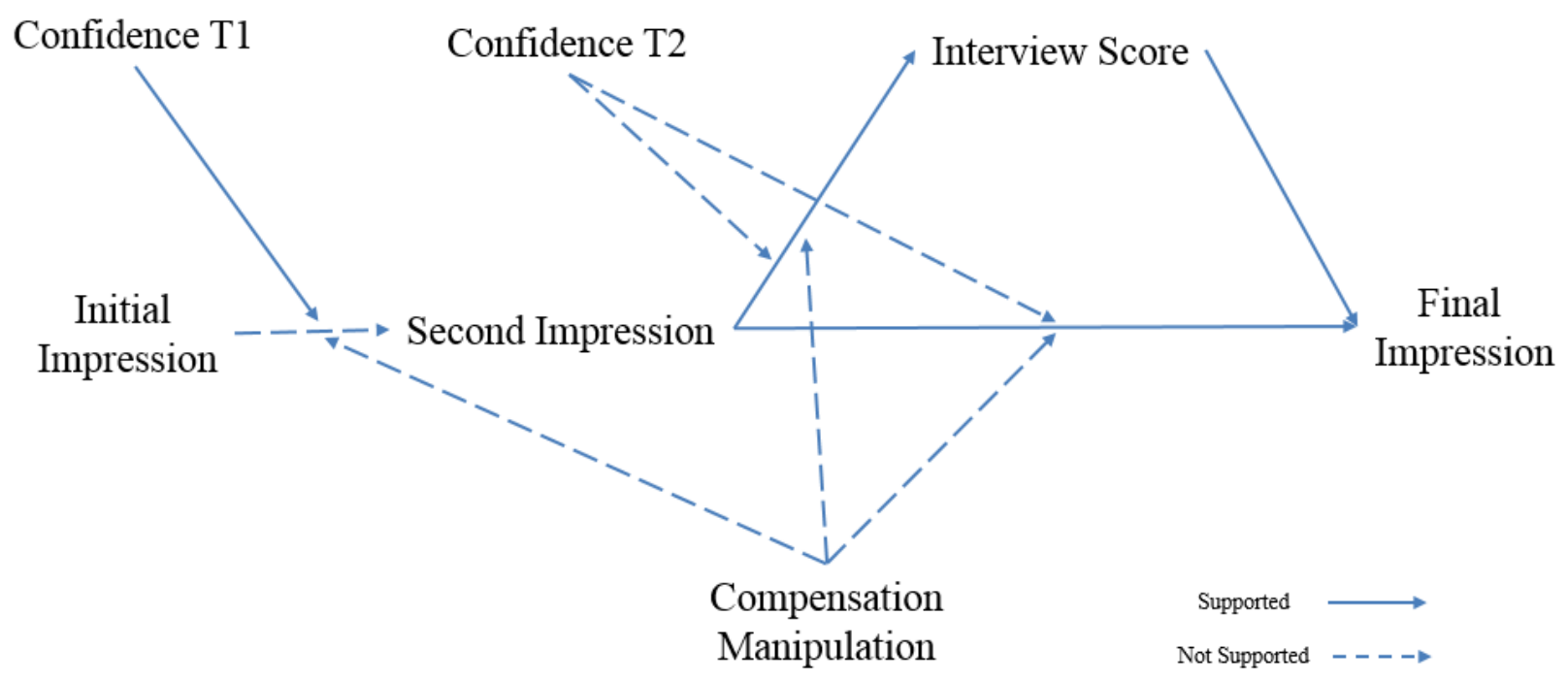

Figure 7. Hypothesis Results 


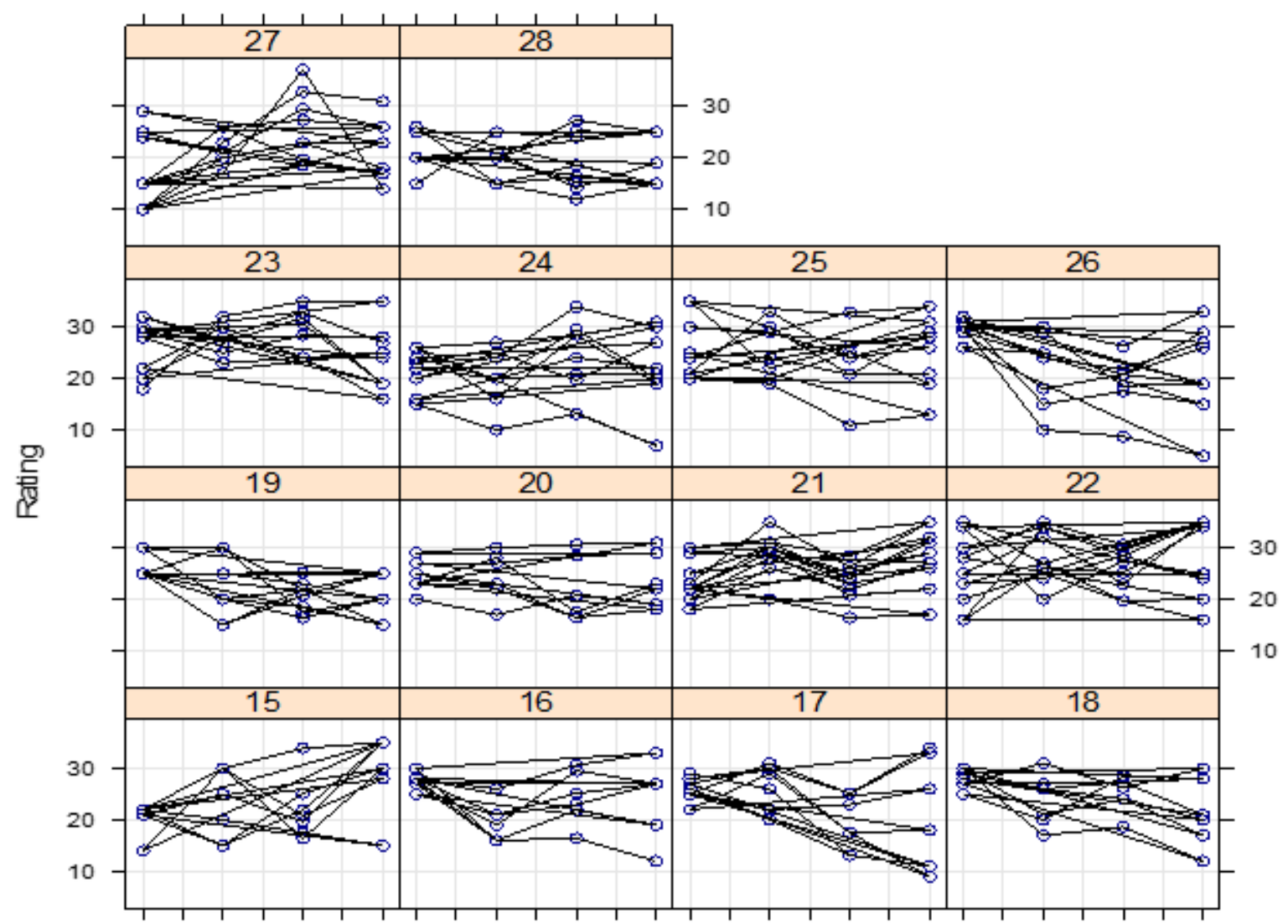

Figure 8. Fixed Compensation Recruiter Rating Stability 


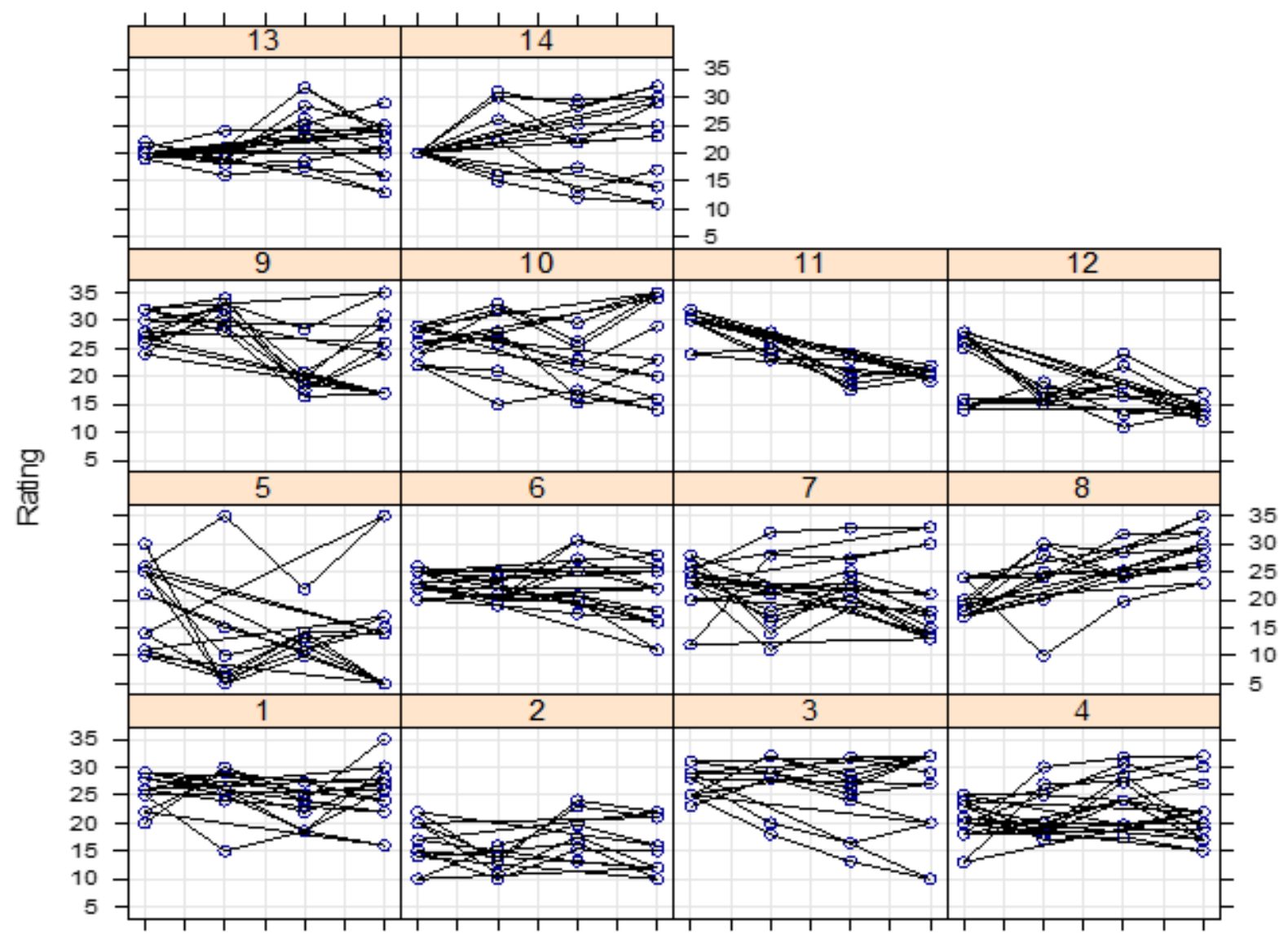

Figure 9. Incentive Compensation Recruiter Rating Stability 


\section{Biographical Sketch}

Andrew M. Carnes is a Doctoral Candidate in Management in the College of Business and Economics at West Virginia University. His research interests include decision making, employee selection, social influence processes, and strategic human resource management. His research in these areas has been published in the Journal of Occupational and Organizational Psychology, Journal of Leadership and Organizational Studies, and Leadership and Organization Development Journal. In addition to his substantive interests, Carnes also has methodological interests in the areas of multilevel phenomena, structural equation modeling, and longitudinal analysis. He was grew up in Gastonia, North Carolina and attended Western Carolina University where he received undergraduate degrees in Construction Management and Business Administration. Later, he attended West Virginia University to obtain a master's degree in Human Resources and Industrial Relations. 